Portland State University

PDXScholar

\title{
Understanding Male Nursing Student Perceptions of the Influence of Gender: A Qualitative Case Study Approach of Students, Faculty, and Administration in a Pacific Northwest Nursing Program
}

Jennifer Anne Anderson

Portland State University

Follow this and additional works at: https://pdxscholar.library.pdx.edu/open_access_etds

Part of the Educational Leadership Commons, Medical Education Commons, and the Nursing Commons

Let us know how access to this document benefits you.

\section{Recommended Citation}

Anderson, Jennifer Anne, "Understanding Male Nursing Student Perceptions of the Influence of Gender: A Qualitative Case Study Approach of Students, Faculty, and Administration in a Pacific Northwest Nursing Program" (2014). Dissertations and Theses. Paper 1935.

https://doi.org/10.15760/etd.1934

This Dissertation is brought to you for free and open access. It has been accepted for inclusion in Dissertations and Theses by an authorized administrator of PDXScholar. Please contact us if we can make this document more accessible: pdxscholar@pdx.edu. 
Understanding Male Nursing Student Perceptions of the Influence of Gender:

A Qualitative Case Study Approach of Students, Faculty, and Administration in a Pacific

Northwest Nursing Program

by

Jennifer Anne Anderson

A dissertation submitted in partial fulfillment of the requirements for the degree of

Doctor of Education

in

Educational Leadership: Postsecondary Education

Dissertation Committee:

Michael J. Smith, Chair

Karen Haley

Toeutu Faaleava

DeLys Ostlund

Portland State University

2014 
(C) 2014 Jennifer Anne Anderson 


\begin{abstract}
In contemporary American society, the nursing profession is predominantly made up of white women. Currently, males make up only 6.8 percent of the three million nursing professionals in the U.S. and they are considered gender minorities within the nursing profession and within nursing education. As gender minorities, male students are identified as experiencing nursing programs and the practice of nursing differently than their female counterparts.
\end{abstract}

The purpose of this single instrumental, within site case study was to explore the learning environment for male nursing students and to investigate the nature of the interactions between nursing faculty and male undergraduate students in a Pacific Northwest medical university nursing program. Data was collected in the educational setting through observations, participant interviews, and document analysis. In addition, this study used Kanter's theoretical framework of tokenism to uncover if male nursing students were perceived as tokens in the educational environment. The findings showed that the faculty-student interactions were largely positive; they also revealed that having other males in the class was an instrumental factor in their positive perception of their educational experience. However, the male nursing students also identified areas of discomfort in the educational setting, specifically when practicing clinical skills with female peers, feeling pressured to volunteer and to expose skin during in-class demonstrations, and anticipating that they would be excluded from certain clinical 
situations. This research indicated that gender differences are present within nursing education and contributed to instances of discomfort for male students. Specific barriers occurred most often when men engaged with female peers and were in clinical settings. These findings provide new insight into when and where men begin to experience gender barriers in the educational environment and are pertinent to understanding the educational environment for men in nursing. Recommendations specifically geared towards assisting students in their first term are suggested for nursing faculty and administrators to ensure that the learning environment is welcoming for men. These recommendations include consciously placing males together in cohort groups and in clinical experiences, reducing instances of visibility and pressure on men in the clinical setting, building faculty awareness of perceived and real barriers for men in the educational setting, and providing faculty with tools to assess and address barriers that are present in the classroom environment. 


\section{ACKNOWLEDGEMENTS}

I am filled with gratitude for my family, friends and colleagues who supported me as I made my way on this educational journey. First to my family, I want to acknowledge my mother, Suzan Midkiff, who instilled in me the value of higher education, has always shown me that she believed in me, and who never doubted that I would succeed. I would also like to acknowledge my step-father, Michael Geigle, who taught me that fatherly love knows no bounds, and who is an infinite well of love and support. Finally, I want to thank my wife, Dr. Sue Orchard, who freely supported me throughout this process, willingly sacrificing shared weekends and activities over the past four years so that I could dedicate time to my studies and who was eternally patient, kind, and encouraging as I made my way along.

I also could not have completed this journey without my educational and professional colleagues, and I would like to thank the faculty and students that participated in this study and granted me access to their learning environment. This dissertation could not have happened without their participation, and I appreciate their willingness to share their educational experience and perspective with me. I would like to thank Dr. Linda Reisser, who allowed me to witness her leadership in action first hand as her intern and who is a great mentor and inspirational leader. I would also like to thank my committee members for their active engagement in this process, as well as my committee chair and advisor, Dr. Michael Smith. Dr. Smith tirelessly provided direction and feedback, taught me to think deeply about this work, and always had an encouraging word to keep me moving forward. Finally, I would like to recognize Dr. Michael Bleich who believed in me, gave 
PERCEPTIONS OF MALE NURSING STUDENTS IN NURSING EDUCATION iv me the opportunity to lead and grow within a complex organization, and whose support and mentorship gave me the inspiration to take my own next steps within higher education. To each and every person, my deepest and sincerest appreciation. 


\section{Table of Contents}

ABSTRACT i

ACKNOWLEDGEMENTS

CHAPTER I: INTRODUCTION

Male Nursing Students And Marginalization: Three Conversations 1

Data Supporting Status OF MEN AS “MinORItiES” IN NuRSing 4

MEN AS A MiNORITY IN THE NURSING PROFESSION

FEMINIZATION OF NURSing AND SOCIO-CUltural Pressures ON MALES 7

Why RETAINING MALES IS IMPORTANT TO NURSING SCHOOL FACULTY AND ADMINISTRATORS 9

SAME OPPORTUNITIES FOR SUCCESS 10

CHALLENGING GENDER STEREOTYPES

Recruiting Men to Manage the Nursing Workforce SHORTAge 12

The Aging Population And Diversifying the Nursing Workforce 14

GENDER BIAS IN NURSING EDUCATION 16

PURPOSE OF THIS STUDY 17

CHAPTER II: LITERATURE REVIEW 20

GENDER STEREOTYPES AS BARRIERS TO THE EDUCATIONAL EXPERIENCE 22

SKEWEd PREFERENCES AND ANTI-MASCULINITy BIAS IN NURSING EDUCATION 24

MEN AS INTRINSICALLY UNABLE TO CARE?

Stigmatizing Male StUdents Use OF Touch 26

FACULTY AS ENFORCERS OF DOMINANT FEMININITIES IN NURSING EDUCATION 28

Role-Strained Male Nursing Students in a Female-Dominated Land 32

TOKENISM AND GROUP DYNAMICS IN NURSING CULTURE 34

$\begin{array}{ll}\text { SUMMARY } & 40\end{array}$

CHAPTER III: RESEARCH METHODOLOGY 45

QuAlitative RESEARCH STRATEgy 46

STUDY DESIGN $\quad 48$

SAMPLING $\quad 50$

METHODS

ACCESS TO THE SITE

PARTICIPANT RECRUITMENT 
PERCEPTIONS OF MALE NURSING STUDENTS IN NURSING EDUCATION Vi

PARTICIPANTS 53

INFORMED CONSENT $\quad 54$

OBSERVATIONS

INTERVIEWS

DOCUMENT ANALYSIS

DEDOOSEC $\quad 58$

DATA ANALYSIS

$\begin{array}{ll}\text { SUMMARY } & 60\end{array}$

CHAPTER IV: FINDINGS

Theoretical Proposition 1: Men Are Isolated From Faculty AND PEERs in NURSING 63

TheORETICAL Proposition 2: MEN ExPERIENCE BARRIERS IN THE EdUCATIONAL ENVIRONMENT $\quad 76$

Theoretical Proposition 3: As Gender Minorities, Men May Not Be as

ACADEMICALLY SUCCESSFUl IN A FEMALE-DOMINATED EDUCATIONAL ENVIRONMENT 85

$\begin{array}{ll}\text { CONCLUSION } & 92\end{array}$

CHAPTER V: DATA ANALYSIS $\quad 94$

NATURE OF INTERACTIONS OF FACULTY AND STUDENTS 96

PATtern 1: Faculty Are A HelpFul, Supportive Resource 96

PATTERn 2: FACUlTy's CAUTiOUS COMMENTS OF SMALL DifFERENCES 99

PATTERN 3: BENEFITS OF EXTROVERSION VERSUS DEFICITS OF INTROVERSION 102

PATTERN 4: SOCIETAL NORMS AND EXPERIENTIAL GAPS 105

SUMMARY OF INTERACTIONS OF FACULTY AND STUDENTS 108

GENDER NEUTRALITY: DESCRIPTIONS, REPRESENTATIONS, AND REFLECTIONS 109

SUMMARY OF GENDER NEUTRALITY 110

KANTER'S THEORY OF TOKENISM: VISIBILITY, POLARIZATION, AND ASSIMILATION 110

VISIBILITY

SUMMARY ON VISIBILITY

POLARIZATION $\quad 121$

SUMMARY OF POLARIZATION

ASSIMILATION 126

CONCLUSION OF KANTER's (1977) PERCEPTIONS OF TOKENISM 129

FACULTY AWARE OF GENDER BARRIERS IN THIS “ESTROGEN-SOAKED” FIELD 130 
PERCEPTIONS OF MALE NURSING STUDENTS IN NURSING EDUCATION vii CHAPTER VI: DISCUSSION AND IMPLICATIONS 139

DiscusSiOn OF THEORETICAL PROPOSITIONS FROM CHAPTER IV 139

Men Were Not Isolated From Faculty and PeERS IN NuRsing SchoOL 140

MEN EXPERIENCE SOME BARRIERS

THE SPOTLIGHT AND ACADEMIC SUCCESS 145

SUMMARY OF THEORETICAL PROPOSITIONS FROM CHAPTER IV 146

DiSCUSSION OF RESEARCH QUESTIONS FROM CHAPTER V 147

FACULTY-STUDENT INTERACTIONS 148

$\begin{array}{ll}\text { GENDER } & 148\end{array}$

TOKENISM AND FACULTY AWARENESS OF GENDER BARRIERS 149

FACULTY AWARENESS OF GENDER BARRIERS 150

RECOMMENDATIONS 152

IMPLICATIONS FOR FUTURE RESEARCH 158

LIMITATIONS 158

$\begin{array}{ll}\text { CONCLUSION } & 159\end{array}$

REFERENCES

$\begin{array}{ll}\text { APPENDICES } & 174\end{array}$

Appendix A: Case Study Design Graphic 174

ApPendix B: All Data Collection Timeline 175

APPENDIX C: EMAILS FOR RECRUITMENT PARTICIPATION 177

APPENDIX D: INTERVIEW QUESTIONS 180

APPENDIX E: CODE GRAPHIC 186 


\section{CHAPTER I: INTRODUCTION}

\section{Male Nursing Students and Marginalization: Three Conversations}

In contemporary American society, the nursing profession is predominantly made up of white women (U.S. Department of Health and Human Services, Health Resources and Services Administration, 2008). In fact, women have dominated the field for most of the past two centuries, and although societal views have changed regarding gender roles and the nursing profession, this female-dominated field continues to be difficult for males to enter. As an administrator in an urban Pacific Northwestern nursing school, I have taken a keen interest in the male student perception that nursing is a female-dominated arena. My interest was further piqued by three interactions I had with male nursing students in our institution. Connected to this interest is the question what do nursing faculty and administrative staff know about how these male students feel? What precisely is their reaction to the issues around male engagement in a female normative profession?

The first interaction occurred with two male students in their second year of our undergraduate nursing program. They asked me how many males were entering into the program that fall? When I told them the number was around 5\% of the new cohort, they looked at each other with a curious, knowing glance that caught my attention and moved me to ask why they were interested in this information. One responded, "It's just different if there are more males in the classroom.” I was struck by the word "different” and intrigued as to what this might mean for them. 
The second interaction took place at an orientation event. The questions posed by the first two men inspired me to query another undergraduate male in our program. To my question about his experience on his first day as a nursing student, he responded, "The cliques are already forming and I feel a little left out.” This can happen to any new student, but I wondered if his status as a male in a program dominated by females played a role in his feeling "left out.” It occurred to me that men in a cohort dominated by women could experience a kind of marginalization similar to women in STEM (science, technology, engineering, and math) programs or underrepresented minorities in predominantly white colleges. For this student, the social isolation was immediate and central to his experience as a nursing student starting on day one of his program.

Finally, during that same academic year, a current male nursing graduate student asked me to help him start a "men in nursing” organization at our campus as a means of support and encouragement for male nursing students. He was seeking support and mentorship for his journey through nursing education and that he had not found it in our institution. All three of these interactions underscored not only the male students' marginalization but also their need for mentorship and instruction about how to navigate the experience of being male nursing students in a female-dominated program. While this group of male nursing students seemed keenly aware of their different status within their peer group, my faculty and administrative colleagues seemed surprisingly unaware.

These male nursing students were seeking greater support and guidance with their gender issues in a female normative program. My male and female faculty and administrative colleagues, on the other hand, gave me a wide range of responses about 
the issue of men in nursing, most barely acknowledging any differences in the learning environment for male students. One faculty member asked, "What are the issues for men in nursing?” Other responses were more ambivalent. One fellow administrator said, “I haven’t given it any thought.” Another stated, “males may be minorities in nursing but they aren’t disadvantaged.” Other statements tended toward accusation: one faculty member said she believed that "the male nursing students tend to be the ringleaders in voicing concerns on behalf of the cohort whenever there is a perceived problem in the program" or the comment by a male nursing faculty member that "men may face discrimination while in school but they are advantaged once they get into the nursing workforce.” This wide range of responses from both male and female nursing faculty and administrators led me to believe that the issues males face in nursing education maybe be perpetually overlooked by faculty and administrators and that these men may suffer in silence.

These revelations occurred within three months that coincided with my first year of doctoral study. During this first year, my classes discussed issues of access, equity, and the factors for student success in higher education; and through the readings, discussions, and writing assignments, I was reminded of these three student interactions. I thought about what could be done to reduce the feelings of isolation that these men might be experiencing in the nursing program. I wondered what their isolation might mean for their future success in our program and further their persistence into the nursing profession. For these reasons, I chose to explore the topic of men in nursing for my dissertation with a particular focus on the educational climate for men in nursing. A 
review of the literature for males in nursing shows that that this topic is timely and critical to the future of addressing the healthcare needs of the American population.

\section{Data Supporting Status of Men as "Minorities" in Nursing}

Currently, males make up only $6.6 \%$ of the three million nursing professionals in the United States (U.S. Department of Health and Human Services, Health Resources and Services Administration, 2008, p. 13). In 2000, the percentage of men in the nursing population was $5.4 \%$ of the overall population, and in 2004 males comprised $5.8 \%$ of the overall nursing population (U.S. Department of Health and Human Services, Health Resources and Services Administration, 2000, p. 8; U.S. Department of Health and Human Services, Health Resources and Services Administration, 2004, p. 13). This limited increase in numbers from $5.4 \%$ to $6.8 \%$ over most of a decade indicates that males are continuing to join the ranks of the nursing population but that parity in the number of males and females in this profession is a long way away. "While more men are choosing nursing than in decades past, men will likely remain the minority in nursing for many years to come” (Dyck, Oliffe, Phinney, \& Garrett, 2009, p. 649).

The low numbers of male nurses is also reflected in the nursing workforce in Oregon. As of 2011, the Oregon State Board of Nursing identified 45,946 people as holding a nursing license through the state board with 35,849 of those employed as nurses (Oregon Health Authority, 2011). Of this group, 10\% identified as male (Oregon Health Authority, 2011, p. 37), a number slightly above the national average. However, the male to female distribution within nursing is still not on a par with the gender distribution 
across America or within the state of Oregon, which is roughly equal. Given the equality of representation, both nationally and in Oregon, we might expect that the gender distribution within nursing would be moving closer to equal as well. Minority can be defined as "a subordinate group whose members have significantly less control or power over their lives than members of a dominant or majority group (Schaefer, 1993, p 10). According to workforce data, men are by definition a minority population within nursing, and men are also a minority population in nursing education from community college to undergraduate programs in four-year colleges and universities on through graduate school. As this problem comes into clearer focus, what are some of the societal and structural factors that shape female domination of the nursing field?

\section{Men as a Minority in the Nursing Profession}

The issue of gender inequities and the differential treatment of a subordinate group have been reviewed in the literature. Kanter (1977) looked at issues of differential treatment in the work place when one gender group makes up a much greater proportion than the other, arguing that "skewed groups," where "there is a large preponderance of one type over another," create situations where members of the minority group are “treated as representatives of their category as symbols rather than individuals” (p. 966). In nursing, the gender imbalance is skewed such that women are in the majority. Kanter (1977) also argued that in situations where the gender balance is skewed, the "group structures shape interaction contexts and influence particular patterns of male-female interaction” (p. 967). Within nursing, these gender interactions may shape the ability of 
males to adapt and cope with members of the dominant group which is inherently important in supporting their continued growth in the profession.

As minorities within the field, the literature has shown that men have less influence within the nursing profession and have more difficulty successfully fulfilling the role or bringing their own ways of knowing and doing into accepted nursing practice (Bartfay, Bartfay, Clow, \& Wu, 2010; Dyck et al., 2009; Kelly, Shoemaker, \& Steele, 1996; Meadus \& Twomey, 2011; Okrainec, 1994). In addition, the nursing literature indicates that male nurses experience nursing programs and the practice of nursing differently from their female counterparts (Anthony, 2004; 2006; Bell-Scriber, 2008; Dyck et al., 2009; O’Lynn, 2004; Yang, Gau, Shiau, Hu, \& Shih, 2004). In particular, the literature has shown that men experience barriers to their practice as nurses both as nursing students and within the profession. These barriers include feelings of isolation, stereotyping, gender discrimination, disenfranchisement from the curriculum, female-focused roles that are difficult for men to relate to or adapt to, and resistance from female patients related to appropriate touching (Anthony, 2006; Denhart, 2008; Evans, 2002; Kulackac, Ozkan, Sucu, \& O'Lynn, 2009; Meadus, 2000; O'Lynn, 2004; Roth \& Coleman, 2008).

Although men, particularly those who are European American (white), are often seen as holding a privileged place in our society, the gender imbalance within nursing education can create a culture that makes it difficult for men as minorities to function on equal footing with their female peers. "Once enrolled, many male students experience nursing schools as places where gender is a significant determinant of the educational environment and where femininities are predominant” (Dyck et al., 2009, p. 649). 
Concerns have been raised that these types of barriers create a "cool climate” (BellScriber, 2008) for men that results in lower retention rates for men in nursing school (McLaughlin, Muldoon, \& Moutray, 2010) and in the nursing profession (Evangelista \& Sims-Giddens, 2008; Gaynor, Gallasch, Yorkston, Stewart, \& Turner, 2006). If we ask why a less than warm learning environment exists for men in nursing schools, the answer may be a history lesson that covers a century of female dominance and social reinforcement in this field.

\section{Feminization of Nursing and Socio-Cultural Pressures on Males}

Societal perceptions that nursing is a female-oriented role have been a major factor in the low numbers of men in the nursing field and continue to exert pressure on those in the nursing field today (Anthony, 2004; Mackintosh, 1997; Poliafico, 1998; Thomas, 1998). From the start, the role of the nurse has been strongly influenced by culture and societal attitudes. As nursing developed in the $19^{\text {th }}$ century, the image of the nurse evolved from that of a servant to that of an educated professional (Andrist, Nicholas, \& Wolf, 2006; Berman, Snyder, Kozier, \& Erb, 2008; Donahue \& Russac, 1985; Judd, Sitzman, \& Davis, 2010; Mackintosh, 1997; Maggs, 1996). Although women were originally considered unequipped for work outside the home, the work of Florence Nightingale and others who followed her created a pathway that gave women across Europe and America access to an education and a livelihood that they did not have previously (Andrist et al.,2006; Berman, et al., 2008; Judd et al., 2010). This new standard of care helped establish nursing as a profession with standardized education and training. In addition, it gave women an accepted role in the profession and helped establish women outside of the 
home, allowing them to contribute beyond their immediate families to their communities (Andrist et al., 2006; Berman et al., 2008; Judd et al., 2010).

As societal acceptance of women in the nursing role grew, nursing itself became strongly identified as women’s work within American popular culture (Andrist et al., 2006; Berman et al., 2008; Judd et al., 2010). For example, women were specifically recruited to participate in World War I and II as nurses (Judd et al., 2010). At the same time, with societal acceptance of women in the nursing role, the characteristics of caring, nurturing, and support that are part of nursing practice became widely seen as inherent feminine qualities (Berman et al., 2008; Andrist et al., 2006; Judd et al., 2010). “Many people, including nurses themselves, believe that being a good nurse is dependent on innate biological characteristics and by inference, stereotypical feminine characteristics” (Jinks, 1993, p. 287). This early connection between nursing and a gender-specific role has persisted in the profession (Thomas, 1998). Female nursing dominance has had a large impact on how the members of the profession are viewed by society and has determined what qualities are seen as integral to fulfilling the nursing role (Evans, 2002; Grady, Stewardson, \& Hall, 2006; Jinks, 2004). While the norms and attitudes that have helped shape the nursing profession have been useful in promoting the education and livelihood of women outside of the home, they also serve to maintain the gender homogeneity of the profession today.

As the profession of nursing has emerged in the $21^{\text {st }}$ century, the social mores linking women to nursing are being challenged, and calls to address the gender imbalance have increased from external agencies. This can be seen in position papers and marketing 
campaigns directed at men (American Assembly of Men in Nursing, 2011; American Association of Colleges of Nursing, 2011; Johnson \& Johnson, 2011). Changes are also occurring within the professional ranks with "increasing numbers of nursing leaders acknowledging male nurses' contributions, both because of their clinical competency and because of day-to-day nursing shortages, particularly in Western societies” (Yang et al., 2004, p. 643). Today, greater value is being placed on gender equality within all professions, especially in those targeting women in STEM fields, and this has created greater awareness of ensuring job roles that are equally represented by both genders (Meadus, 2000; Vogt, Hocevar, \& Hagedorn, 2007; Wingfield, 2009). As gender equality has become more of a societal expectation, women have made strides in attaining educational and professional roles in fields from which they were historically barred (Meadus, 2000; Vogt et al., 2007; Wingfield, 2009). However, the slow growth of male entry into the nursing field indicates either that gender equality within nursing may not yet be fully embraced or that the reasons why men need to play a bigger role in the nursing profession have yet to be convincingly articulated.

\section{Why Retaining Males is Important to Nursing School Faculty and Administrators}

The ability to retain and graduate men from nursing programs is critical to increasing the overall number of males in the nursing profession, which will help diversify the nursing workforce across the nation. This diversity is a strategic goal for national nursing and health-related organizations like the American Association of Colleges of Nursing (AACN), Institute of Medicine (IOM), and the Sullivan Commission. 
Nursing education is critical to the formation and training of professional nurses, and this link between the educational system and the profession has caused national leaders to look to nursing schools to educate more male students. This external pressure to diversify the nursing student body has resulted in additional scrutiny from external sources about the barriers men and underrepresented students face in predominantly female and white nursing education programs (Anthony, 2004; Bartfay et al., 2010; BellScriber, 2008; Dyck et al., 2009; Kelly et al., 1996; Meadus \& Twomey, 2011; Okrainec, 1994; O’Lynn, 2004; Poliafico, 1998; Yang et al., 2003). As such, it is important for nursing faculty and administrators to address the barriers males have identified in nursing school in order to 1) ensure that nursing schools are providing the same opportunity for educational and professional success to all students; 2) challenge gender stereotypes that continue to uphold the societal view that nursing is “women’s work” and help increase the visibility within our culture that nursing is a skilled and technical field that is not limited based on gender; 3) address the nursing workforce shortage by recruiting talented and diverse males; 4) meet the diverse needs of the aging population by diversifying the nursing workforce; and 5) challenge gender stereotypes embedded in nursing education.

\section{Same Opportunities for Success}

Today’s nurses require a high level of education, technical expertise, and dedication in order to fulfill their jobs. Students pay thousands of dollars to receive an education that leads to the successful pursuit of a nursing career. In addition, the nursing profession is counting on nurse educators to educate and train future nurses who are fully capable and competent to meet the needs of a diverse and changing patient population. "The objective 
for schools of nursing is the creation of both an educational community and a professional practice environment that incorporate the diverse perspectives of the many constituencies whom they serve” (AACN, 2011, p.1). Nurse faculty and administrators have responsibilities for ensuring that all qualified students are given the same opportunities for successfully obtaining the knowledge and skills necessary to become professional nurses (Anthony, 2004; Bell-Scriber, 2008; Bowen, 1977; Meadus \& Twomey, 2011). If there are institutional practices or procedures that negatively impact the learning environment of male nursing students, it is critical that nursing faculty and administrators take the necessary steps to understand and resolve these issues for the benefit of male students and female students who together represent the future of nursing practice.

\section{Challenging Gender Stereotypes}

As noted earlier, gender stereotypes related to job roles exist within the greater American culture and can be found in nursing culture to this day (Anthony, 2004, 2006; Bartfay et al., 2010; Bell-Scriber, 2008; Meadus, 2000; Meadus \& Twomey, 2011). The literature notes that men in nursing experience role strain (Bartfay et al., 2010) in the form of gender stereotypes, which can lead to men having to explain why they chose nursing instead of becoming a medical doctor. Such pressure results in male nurses having to go out of their way to reinforce their masculinity when working with patients and peers (Anthony, 2004, 2006; Bartfay et al., 2010; Bell-Scriber, 2008; Meadus, 2000; Meadus \& Twomey, 2011). Additionally, men are perceived by their female peers as being less caring or compassionate and can sometimes be seen as "more confrontational” 
than others in the nursing profession (Bartfay et al., 2010; Dyck et al., 2009; Meadus \& Twomey, 2011).

Left unchallenged, these stereotypes often serve to uphold current perceptions that nursing is a role that is "natural” for women, an idea that perpetuates the myth that nursing is women's work and that has a chilling effect on men entering and working in the profession (Bartfay et al., 2010; Kelly et al., 1996). Ultimately, the goal for increasing the number of men in the nursing field is to help change gender roles such that the most qualified individuals will be drawn to the profession regardless of gender. Nursing faculty and administrators have an inherent interest in ensuring that gender bias and stereotypes are minimized in nursing schools in order to provide equitable learning situations for all students as well as in creating a nursing workforce that reflects the larger population.

\section{Recruiting Men to Manage the Nursing Workforce Shortage}

In addition to challenging gender stereotypes and diversifying the national workforce, there is a need to attract more men into nursing because workforce data from early 2000 has shown that the nursing workforce is aging and large numbers of nurses are preparing for retirement (Jeffreys, 2007; Meadus, 2000; U.S. Department of Health and Human Services, Health Resources and Services Administration, 2008; Williams, 2010). In fact, in the late 2000s it was projected that $16.2 \%$ of RNs from age 50 to 54 stated that they were looking to retire from nursing in the near future (U.S. Department of Health and Human Services, Health Resources and Services Administration, 2008, p. 8). This exodus of senior nursing professionals has the potential to create a deficit of nurses, often 
referred to as "the nursing shortage” (Crow, Smith, \& Hartman, 2005) and that the “shortage is estimated to double from 6 percent in 2000 to 12 percent in 2010” (2005, p. 337). The National Sample Survey of Registered Nurses report (U.S. Department of Health and Human Services, Health Resources and Services Administration, 2008) supported this claim showing that "approximately 291,000 RNs allowed their U.S. licenses to lapse, possibly indicating that the substantial retirements that have been anticipated may have begun” (p. xxvii). In addition, the Oregon Center for Nursing identified that "an additional 5,947 replacement openings will be created by RNs leaving the workforce” between 2008 and 2018 (Oregon Center for Nursing, 2011, p. 2), further indicating that the local nursing workforce is beginning to be impacted by nursing shortages. The combined factors of increased patient access to medical care and an aging population are raising the demand for health care and nursing services across the nation (U.S. Department of Health and Human Services, Health Resources and Services Administration, 2008). The need for nursing is being driven by the needs of the aging "Baby Boomer” generation and by the increase of new patients accessing care through National Health Care Reform (Institute of Medicine, 2001) and the recently passed Affordable Care Act (U.S. House of Representatives, 2010). With aging nurses retiring and the need for nursing services increasing, the educational and professional opportunities for those choosing nursing as a career will continue to grow. In fact, U.S. Bureau of Labor and Statistics project that the nursing workforce will grow to 3.6 million by the year 2018 and that roles in health care and social services will have "the second largest increase among all sectors” (Woods, 2009, p. 56). This nursing shortage cannot be 
filled by women alone. O’Lynn (2004) contended, “if men entered the profession at the same rate as women today, there would be no nursing shortage” (p. 230).

At the same time, there are no guarantees that American women will continue to pursue nursing as a professional option in adequate numbers since other more prestigious and lucrative medical career options are becoming available. Women have more educational and professional choices than ever, and our society can no longer expect that women will select nursing because it is one of the only options available to them. Therefore, as women select from the various career options that they have, the ability to fill the nursing ranks through women alone is not a sustainable option. The only way that the nursing workforce will be able to keep up with the societal demand for nurses is to attract and retain men and women in equal numbers (Kippenbrock, 1990; Meadus, 2000). It is no longer acceptable to rely on one gender to fill the need that exists within the professional nursing ranks nor would this continued homogeneity within the workforce help meet the needs of a diverse population and an increasingly technical field. The ability to replace the retiring nursing workforce will require that people from all creeds and colors, and both genders be encouraged and welcomed into the profession.

\section{The Aging Population and Diversifying the Nursing Workforce}

The American population is becoming increasingly diverse with 34\% of the total U.S. population identifying as racially or ethnically non-White (U.S. Department of Health and Human Services, Health Resources and Services Administration, 2008). As the demographics of the American population continue to shift, nurses are being called upon to more effectively engage with and support linguistically, culturally, and ethnically 
diverse patients (Buerhaus, 1999; Fleming, Berkowitz, \& Cheadle, 2005, Sullivan

Commission, 2010). In today’s culture, it is argued that having a largely white, female nursing workforce fails to support the needs of an increasingly diverse patient population (Institute of Medicine, 2004). In fact, Sullivan (2005) noted that “today’s physicians, nurses, and dentists have too little resemblance to the diverse populations they serve, leaving many Americans feeling excluded by a system that seems distant and uncaring” (p. 6).

As part of these often volatile demographic shifts, health care providers are being called upon to engage with and support underserved communities with greater effectiveness (Buerhaus, 1999). In addition, arguments are being made that having a workforce that is more diverse and representative of the current population nationwide helps reduce health disparities for underserved communities and provides a nursing workforce that is more reflective of the current population (Fleming et al., 2005; Nnedu, 2009). It is critical that nursing faculty and administrators pay attention to the changing needs of America's patient population in order to best prepare students for their future role within the nursing workforce. For this reason, the AACN has issued a number of papers over the past decade highlighting the need for nursing institutions to increase the diversity of their student cohorts (AACN, 1997, 2011).

Since males are minorities in nursing, increasing the number of males in nursing education will help contribute to the national goals of diversifying the nursing workforce. In addition, nursing schools have a responsibility to help prepare a nursing workforce that is ready to meet the needs of our diverse patient population, which includes diversity in 
gender as well as race, culture, and ethnicity. The watchful eyes of national professional nursing organizations will continue to pressure nursing schools to be accountable for ensuring that they educate a diverse student body by enforcing accreditation standards, state and federal oversight, and grant funding. For these reasons, nursing faculty and administrators need to be concerned with the retention of males in their nursing programs, especially those from diverse ethnic, racial, cultural, and linguistic backgrounds.

\section{Gender Bias in Nursing Education}

While the issues for men in nursing can be seen as stemming from the feminization of the nursing role over the past hundred years, successfully addressing potential barriers for men in nursing today is essential to meeting the goals outlined above. Nursing schools and educational programs have played a role in the standardization and professionalism of nursing practice since Florence Nightingale opened the first nursing school in 1860 (Judd et al., 2010). Since then, the role of the nurse has transformed from that of a handmaiden into a trusted and lauded professional, a career where best practices are standardized and entry into the profession is based on academic and professional abilities (Judd et al., 2010). Today, the role of nursing education cannot be understated as a major player in the preparation and socialization of nurses into the professional role. Streubert (1994) stated that "through provision of multiple activities that expose nursing students to diverse issues, such as gender, nurse educators assist students to fulfill their roles as holistic care providers” (1994, p. 32). It is clear that nurse educators are not only responsible for preparing students to provide 
primary care to people in need but they also have a responsibility to prepare nurses to respond to the changing needs of the patient population in part by diversifying the workforce (Anthony, 2004).

Meadus and Twomey (2011) found that "gender bias is not only limited to nursing texts but [is] found within the classroom and clinical areas as well” (p. 270). The use of feminine pronouns in nursing texts and by faculty is one example of such gender bias. Bell-Scriber (2008) found that faculty members unwittingly create an uncomfortable environment for men in their classrooms by displaying an “...unsupportive (cooling) factor [in the form of] nurse educators' characteristics and behaviors towards men in the classroom” (p. 146). Dyck et al. (2009) noted that the environment was one "where men saw themselves as accommodated but not integrated” (p. 649). These studies demonstrate that men identify the educational environment as a key factor in their satisfaction or dissatisfaction with the nursing field, which suggests that nurse education could greatly improve this climate by changing their pedagogical-andragogical practice.

\section{Purpose of This Study}

Throughout the past two decades, the nursing profession has developed beyond the dominant perspective that nursing is women's work (Anthony, 2006; Meadus \& Twomey, 2011). As the profession seeks to meet the needs of an aging and diverse patient population as well as fill the ranks of the retiring nursing workforce, the old paradigm that the nursing profession is solely for women has been put aside. However, even with targeted recruitment activities, the increase of men in nursing has been slow. Once admitted to nursing school, men experience barriers to their academic success. 
Given that gender bias still appears to exist in nursing education, the time is ripe for nursing faculty and administrators to learn about and address barriers to male nursing student success in nursing school. Indeed, the role of nursing faculty in understanding, addressing, and alleviating gender bias and gender role stereotypes will help create a warmer climate for male nursing students. Kenny (2002) stated that "nursing faculty hold a power that can be effective in liberating students from culturally constructed and constrictive roles” (p. 323). As per the call to continue increasing the diversity of the America’s nursing workforce, the scrutiny on the barriers faced by men in nursing will increase. It is time for nursing faculty and administrators to challenge the perpetuation of the conceptualization of “nursing as women's work” while providing teaching and learning strategies that can meet the diverse needs of both genders (Anthony, 2004). Toward this effort, it is imperative that nursing faculty and administrators be fully cognizant of the many needs of male nursing students.

This study will inform nurse education practice by identifying how nursing faculty engage with male nursing students in the classroom and exploring how faculty engagement influences students educational success. This study will be guided by the following research questions: 1) What are the perceived and real barriers that males face while in nursing school? 2) In what ways do faculty attitudes and perceptions about male expressions of the fundamental nursing characteristics such as caring, nurturing, and service impact the retention of male nursing students? 3) How can administrators identify the needs of male nursing students while providing the best ways to serve and support 
them so that they can be academically successful as well as integrated smoothly into the nursing profession?

This research aims to assess the educational climate for male nursing students identify potential barriers that men may experience in the classroom setting, identify how these potential barriers impact male student academic success, and learn more about faculty and student perceptions about men in the nursing role. 


\section{CHAPTER II: LITERATURE REVIEW}

The transition into nursing school can be difficult for men. The scholarly literature in nursing suggests that once enrolled in nursing programs, men experience feelings of isolation, feel excluded from the curriculum because of a lack of genderneutral or gender-related examples, drop out at higher rates than their female peers, and have few male role models to identify with (Kulackac et al., 2009; McLaughlin et al., 2010; O'Lynn, 2004; Rankin \& Reason, 2005; Smith, 2006; Smith, Crow, \& Hartman, 2007).

The literature also shows that males face challenges in the form of stereotypes from peers as well as from direct and indirect messages from faculty about nursing culture, which pressures them to conform to the more feminine characteristics associated with nursing (Crow et al., 2005; O'Lynn, 2004; Roth \& Coleman, 2008; Smith, 2006). The overwhelming message delivered by the scholarship is that male nursing students' experience is isolating and similar to the experiences of other marginalized groups. This chapter reviews how barriers for men in nursing are composed of any number of separate experiences or conditions. Specifically, the chapter addresses the impact and influence of gender stereotypes on male nurses. Likewise, it uncovers the influence of skewed feminine preference and anti-masculinity bias in nursing education along with the limiting impact of the widely held assumption that men are intrinsically unable to exhibit the nursing practice of caring. Connected to the issue of caring, the review explores how feminized nursing norms stigmatize male nursing students use of touch and how nursing 
faculty are the guardians of feminization in the nursing profession. Finally, the role strain of being a male nursing student in a foreign feminine land is touched on as well as how the dynamic of tokenism has been used to describe the current situation for men enrolled in nursing education programs.

\section{Social Isolation Defined}

Social isolation is a common experience felt by members of underrepresented groups when faced with a new dominant culture group (Bell-Scriber, 2008; Fairhurst \& Snavely, 1983a, 1983b; Kelly et al., 1996; Kulackac et al., 2009). A number of studies have identified that men experience loneliness and feel socially isolated from their peers and faculty members while in nursing school (Bell-Scriber, 2008; Holyaoke, 2000; Kelly et al., 1996; Kulackac et al., 2009). Kelly, Shoemaker, and Steele (1996) conducted a focus-group study of 18 current male and female nursing students centered on the students’ perception of their educational experience. The strongest finding of this study was that social isolation was identified by participants, especially the men, who indicated that they were not automatically included by their female peers in formal or informal conversations in the classroom. They also had to actively initiate conversations with their female peers in order to be included (Kelly et al., 1996).

This sense of not being included in student activities was also identified by O' Lynn (2004) in a study in which 111 male nursing students responded to a survey designed to measure the level of "male friendliness" (p. 229) within the educational setting. O’Lynn found that $83.6 \%$ of the 111 respondents indicated that "not being invited to all student activities” was a significant barrier in the educational setting (p. 233). In a 
qualitative case study that examined the educational climate for new nursing students, Bell-Scriber (2008) found that the four male nursing students experienced differential treatment in the form of heightened awareness from others and prejudiced comments from faculty during class time, which contributed to feelings of isolation in their nursing program. In a literature review of research evaluating influences on male nursing students, Stott (2003) similarly found that "nurse educators have a tendency to isolate male nursing students both in the educational and clinical setting” (p. 91). In a focus group study of 18 male nursing students, Kelly et al. (1996) found that male participants perceived that their classroom experiences and clinical assignments were different than those of their female peers. For example, men were only allowed to treat male patients during health assessment rotations, while at the same time they were excluded from participation in labor and delivery clinics. The men agreed that being the only male in a clinical environment made partnering exercises difficult (Kelly et al., 1996). Finally, a number of researchers attributed the lack of male nursing faculty and male nurses as a contributing factor in the feelings of isolation among male nursing students (Anthony, 2006; Bartfay et al., 2010; Lerardi, Fitzgerald, \& Holland, 2010; Meadus \& Twomey, 2011; O'Lynn, 2004). Through these examples, it becomes clear that social isolation is a factor in the educational experience for men in nursing.

\section{Gender Stereotypes as Barriers to the Educational Experience}

Social isolation could also be an expression of gender bias or stereotyping where the members of the dominant group refrain from interacting with members of the minority group based on their preconceived ideas about whether an individual fits in with 
their group. The literature has reiterated that men in nursing encounter stereotypes and gender bias from peers, faculty, and patients in nursing school (Anthony, 2004, 2006; Dyck et al., 2009; Evans, 2002; Evans \& Frank, 2003; Jinks, 1993; Kermode, 2006; Lerardi et al., 2010; Poliafico, 1998; Roth \& Coleman, 2008). For example, as a result of stereotypes, men reported that they are expected to take on more physical roles with lifting or moving patients and often endure assumptions about their sexuality (Leradi et al., 2010; O’Lynn, 2004; Smith, 2006;). In addition, male nurses often encounter sexual stereotypes where patients and peers view them as sexual aggressors regardless of sexual identity or assume that they identify as gay (Evans, 2002). These types of preconceived judgments by patients can make it difficult for male nurses to interact with the patient, provide care, or otherwise show the type of caring and empathy that is often associated with the nursing role. Evans (2002) argued that "gender stereotypes create discomfort and suspicion on the part of patients” (p. 442), which helps create a tense working environment for male nursing students. Finally, men are assumed to be leaders in more influential or powerful positions and may be judged for not holding a more influential position. Leradi et al., (2010) conducted a study of 7 male nursing students, which found that these students were often misidentified as doctors by patients; similarly, Meadus and Twomey (2011) found that "several of the men students described in their narratives how they were seen as 'doctors' based upon their gender only and felt the need to continue to identify themselves as a student nurse” (p. 275).

Research shows that being misidentified as a doctor has the two-pronged result of not only pointing out to the male nurse that he is not fitting in with the culturally accepted 
gender roles for males in medicine, but that he also has to explain and define himself as being part of a group where he is not automatically perceived to fit or that is perceived as a lower social status. Male nursing students have reported that the resulting emotional fatigue from constant explanation contributed to their low morale (U.S. House of Representatives, 2010). Stereotyping and gender bias work hand in hand to create a less than inclusive and isolating experience for male nursing students.

\section{Skewed Preferences and Anti-Masculinity Bias in Nursing Education}

Bias also occurs in nursing education with the all-too-common assumptions that masculinity is antithetical to the nursing profession. This type of gender bias forces male nursing students to react in unhealthy ways by exhibiting hyper masculinity or conforming to feminine approaches in spite of the individual qualities that they bring to the nursing role. This kind of anti-masculine gender bias is identified as a "skewed preference” for one gender over another (Anthony, 2004). In an overview of the nursing literature, Anthony (2004) identified anti-masculine gender bias as a consistent theme within nursing education and pointed to the lack of historical examples of men in nursing texts. This bias also manifests itself in differing expectations of male nursing students in clinical rotations, differing expectations for male nursing students in their ability to exhibit core nursing professional concepts like caring, and a heightened awareness of a man's inability to achieve nursing norms within educational settings (Alfred, Yarbrough, Martin, \& Garcia, 2011; Grady et al., 2006; Paterson, Tschikota, Crawford, Saydak, Venkatesh, \& Aronowitz, 1996). Anthony’s findings were also consistent with a literature review by Roth and Coleman (2008) that was designed to "describe the perceived or real 
barriers to men seeking a career in nursing” (p. 149). In this review, Roth and Coleman (2008) found that the feminine image of nursing within greater society and the perception of nursing as a female occupation were shared by the majority of students because of "the absence of the historical contributions [and participation of male] nurses from nursing texts [along with] the ubiquitous use of the feminine pronoun 'she' when referring to nurses in nursing schools and hospitals” (p. 149). Additionally, environmental barriers exist in nursing educational programs or current nursing professional practice that present potential barriers to attracting men into the nursing field (Roth \& Coleman, 2008, p. 149).

\section{Men as Intrinsically Unable to Care?}

Often in the research on nursing or nursing education, male students reported that they were perceived as not caring or nurturing enough to be good nurses (Leradi et al., 2010; O’Lynn, 2004; Smith, 2006). Anti-masculine gender stereotyping can also be found in the preconceived ideas that students (both male and female) bring with them into the classroom. For years, the nursing profession has been strongly associated with the qualities of caring and nurturing. Feminine attributes have historically been identified in our society as being uniquely suited to women, who are seen as the only gender truly qualified for this caring and nurturing role (Smith, 2006). The expectation that only women can fill the role of a nurse is deeply ingrained into our social consciousness and can create barriers for others who fill the role but who do not meet those societal expectations (Mackintosh, 1997).

Indeed, in a questionnaire of gender stereotypes for 100 new male and female nursing students, Jinks (1993) found that “79\% of the respondents felt that women would 
make better nurses" and that "83\% of the students felt women were more affectionate and caring than men” (p. 291). In a comparative survey assessing the perception of sexism and gender discrimination across nursing and non-nursing programs, Kermode (2006) found that "nursing students reported significantly higher levels of 'feminist bias' and ‘discrimination' in their program than did non-nursing students” (p. 71). In another quantitative study, Bartfay, Bartfay, Chow, and Wu (2010) surveyed 149 male and female nursing and non-nursing students to "investigate societal attitudes and perceptions towards male nurses enrolled in school of nursing” (p. 1). Their results found that "both female nursing and non-nursing students were found to have less favorable perceptions and negative attitudes towards male nursing students” (p. 5). More importantly, they found that a portion of the male respondents indicated that "they would not encourage a male family member to pursue nursing as a career choice” and that "men suffer from high levels of role strain” (Bartfay et al., 2010, p. 5).

\section{Stigmatizing Male Students Use of Touch}

If we assume that expressing care and concern are fundamental to the role of a nurse, the continued perception that men are not fit for the nursing role may be due to the different tactics that men traditionally use when expressing care to others. Evans (2002) interviewed eight male nurses about their perceptions of gender relations in the workplace; the men perceived themselves to be “cautious caregivers” (p. 443). The participants in that study indicated that men have their own ways of expressing core nursing concepts and said that “men and women nurses' caring styles were not the same” (p. 443). Evans also found that men used "humor and camaraderie" as their ways of 
expressing caring with patients (p. 443) while the use of touch was most often used as an expression of caring by women. One participant of that study indicated that the use of touch as an expression of caring “did not come naturally to them as men” (p. 443) and that men tended to use caution when the patient was a woman or a teenager so as not to be accused of making an unwanted sexual advance.

Harding, North, and Perkins (2008) also explored how gender stereotypes affect the use of touch by male nurses in a study of 18 male nursing students. The subjects indicated that patients refused their care and that they protected themselves from patient accusations of inappropriate touching; as a result, they had difficulty in relating to patients through the use of touch. The findings from these studies suggest that men are hesitant and outright fearful of using touch as a key form of caring within their nursing practice. This further suggests that feminine gender norms stigmatize male nurses' use of touch, thus playing a large role in creating professional obstacles while altering the way that male nurses practice. This is a critical finding because male students believe that they are encouraged by their faculty to exhibit core concepts of nursing in a feminized way while there is little to no evidence that males are supported in showing these same core concepts in their own masculine way. This may mean that there is a lack of awareness by nursing faculty about how to facilitate or promote appropriate ways of showing core nursing concepts from a male perspective.

The literature clearly exposes the impact of social isolation and anti-masculine gender stereotypes experienced by men in nursing education. Normative femininity contributes to males' heightened sense of invisibility within the educational setting while 
reinforcing their status as a gender minority within the nursing profession. The stereotypes and anti-masculine gender bias found in these studies highlight the barriers within the educational environment that can be challenging for men to successfully navigate. It is critical to address the barriers that men face within nursing education in order to support the learning needs of the growing number of men joining nursing programs today, to remove discrimination and stereotypes from the learning environment, and to support the learning and skill building of all students. However, it cannot be assumed that as gender minorities in nursing, male students can successfully navigate or overcome the biases found within nursing education on their own nor should it be seen as unimportant for males to have an educational experience where gender bias and discrimination do not exist. While there is a growing body of literature that identifies the gender bias and discrimination found in nursing education, the role of faculty in maintaining or challenging these existing issues is currently unknown. For these reasons, the role of nursing faculty as the gatekeepers to the knowledge, skills, and cultural norms within nursing education needs to be examined.

\section{Faculty as Enforcers of Dominant Femininities in Nursing Education}

As professionals, nurses have a shared understanding of their role because they use the same technical knowledge, educational language, professional expertise, and work functions. As a group, nurses can be seen as having their own cultural norms and expectations. Culture is defined by Ting-Toomey (1999) as “a complex frame of reference that consists of patterns of traditions, beliefs, values, norms, symbols, and meanings that are shared to varying degrees by interacting members of a community (p. 
10). Becoming a nurse requires rigorous education and evaluation by faculty members who are nurses themselves. Because students are not able to become nurses and enter the profession without specific education and evaluation from nurses, the role of faculty in preparing future nurses is extremely important.

Nursing faculty are primarily responsible for passing on the dominant cultural norms of the nursing profession to nursing students. In addition, nursing programs not only prepare students for the types of clinical skills that they will need in order to effectively carry out their work functions, they also help students adjust to the inter- and intra-personal aspects of the role. "Nursing schools teach the language of the profession, teach 'fitting in,' thinking 'as nurses think' and to reflect and adopt the 'right' behaviors based on dominant nursing norms” (Love, 2010, p. 343). In essence, faculty can be seen as gatekeepers that uphold the dominant values within the nursing culture and seek to pass on those values to those nursing students who gain their approval. Johnson and Christensen (2012) observed that "cultures are maintained over time through socialization and a social-sanctioning process through which members of a culture stigmatize people who break group norms and praise and associate with the people who follow the appropriate cultural norms” (p. 389).

Given that the majority of nurses are white women, normalizing students into the role of "the nurse" is typically done from the female perspective (Anthony, 2006; O’Lynn, 2009; Meadus \& Twomey, 2011). In fact, scholars believe that "nursing education programs largely are based on a female worldview” (Meadus \& Twomey, 2011, p. 269). The dominance of white, female faculty members, along with a curriculum 
that was developed from a feminine vantage point, creates an environment that may be uncomfortable for men to enter. Standards and expectations that are expected from male students and seen as "normal” by the dominant female group may be foreign or unexpected to males (Bell-Scriber, 2008; Dyck et al., 2009; Kermode, 2006; O'Lynn, 2004). As gender minorities that do not automatically conform to the feminized culture within nursing, "men continue to be outsiders both demographically and within dominant nursing cultures” (Dyck et al., 2009, p. 649).

The relationship between male nursing students and nursing faculty members was evaluated in an ethnographic study of 6 male nursing students and 6 female nursing faculty by Dyck, Oliffe, Phinney, and Garrett (2009). The male student participants said that the faculty had a heightened sense of awareness of who they were, tended to ask them to speak or act as a representative for their gender, and that in class "instructors assumed that all students were familiar with female anatomy and embodied experiences” (652). Faculty participants indicated that they had to watch that they weren't "making an assumption that they're all women” when teaching (p.652). The researchers also found that in response to the “dominant femininities” within the nursing culture, male students "tended to respond to (and even possibly exaggerate) traditional masculinity by speaking up, making jokes, leading, seeking to protect women students, and routinely asserting themselves” (p. 652). The presence of conflicting masculine and feminine attributes led these researchers to conclude that "nursing education is highly gender differentiated, and that masculinities and gender relations appear to influence men’s roles in the nursing classroom and the cultures of nursing education in general” (Dyck et al., 2009, p. 652). 
Grady, Stewardson, and Hall (2008) also explored faculty perceptions of caring in male nursing students through semi-structured interviews in a phenomenological study. Participants in this study identified that men showed caring differently than women did and that some men needed additional help with expressing caring to the satisfaction of their faculty members. In addition, “female nursing students were held up as the frame of reference against which male nursing student caring was judged” (Grady et al., 2008; p. 321). By seeing the female way of caring as the right or correct way of exhibiting this core skill, nursing faculty may be knowingly or unknowingly strengthening the feminization of this core concept and making it difficult to see how male ways of showing caring may be different but beneficial to nursing practice. However, despite the fact that men may express caring differently than women, the faculty respondents appeared to have little awareness that differences in gender socialization may make some expected ways of expressing caring difficult for male students.

In fact, research has found that men are often expected to conform to feminine ways of expressing core nursing concepts and that these expectations may be difficult for men to emulate. Gender bias and stereotyping are also shown in nursing education when nursing instructors perpetuate feminine ways of knowing or doing as the only right way for students to exhibit an expected behavior or skill set. If we assume that faculty set the expectations for what is appropriate behavior when caring for a patient then it is important to look at the norm to which that standard is set. Paterson et al. (1996), looked at his question by investigating the "lived experience of male nursing students as they learned to care as nurses” (p. 27). In this study using interviews and narratives from 20 
male nursing students the authors found that caring is more often expected to be demonstrated in a feminine manner (1996). One participant indicated that in order to show caring the way that he was expected to, “I'd have to become less of a man. I'd have to act like a woman, not a man” (Paterson et al., 1996, p. 30). Although gender differences in learning how to care were identified in this study, it did not explore whether having to demonstrate a feminized way of caring hindered the student's ability to be successful in the program. However, the idea that men exhibit care differently was explored in a study of 8 practicing male nurses. Evans (2002) found that male nurses did describe male ways of caring as being different from those of their female peers. One participant indicated that men "have our ways of getting it [caring] across without putting that female bent or lean on it" and that women showed caring in a more "touchy feelie" manner (Evans, 2002, p. 443). These studies taken together show that men are expected to adopt the norms and values of the dominant feminine view and may not have their own gender-appropriate ways of expressing nursing core concepts validated or approved. The consequence is that men are expected to adopt behaviors that are not necessarily congruent with their individual identities, which leads to discomfort and role strain.

\section{Role-Strained Male Nursing Students in a Female-Dominated Land}

Role strain has been defined as "when an individual is likely to experience tension in coping with the requirements of incompatible roles” (Jary \& Jary, 1991, p. 538). For men in nursing, role strain can occur when they are perceived by faculty and peers to occupy a female role incongruent with their masculine gender, when feminine ways of knowing are perceived as the only right way, or when the men encounter gender 
stereotypes that minimize their individual contribution (Bartfay et al., 2010; Bell-Scriber, 2008; Brady \& Sherrod, 2003; Stott, 2003).

Role strain was also studied by Okrainec (1994), whose qualitative work sought to "examine the perceptions of nursing education by male nursing students" in studies where women “equal to the males by type and years of nursing program were included for comparative purposes” (p. 94). In findings that seemed to contradict those of other scholars, Okrainec showed that both men and women believe that women have more natural aptitude for nursing but in a rather interesting twist, that men were slightly more satisfied than their female peers with their relationship with nursing instructors. Okrainec concluded that role strain was not in evidence for the male participants of this study and that "relatively few significant differences were found to exist between male and female nursing students” (p. 106). However, a later study by McLaughlin, Muldoon, and Moutray (2009) found that role strain and gender role stereotyping did exist in a quantitative, longitudinal study reviewing the retention of male and female students where "males were more likely to withdraw from their course than females" (p. 30). The authors cited role strain as a factor in their conclusion that "the female-dominated nature of nursing, prevalent stereotypes and gender bias inherent in the nursing education seem to make this an uncomfortable place for males and those with less gendered typed views" (p. 30). In the study by Bartfay et al. (2010), the authors found that "men suffer from high levels of role strain” (p. 5) and suggested that images within our popular culture help create an environment where male nurses are viewed as “oddities” by society at large (p. 5). 
Although there is no agreement in the literature about whether role strain exists for men in nursing, the idea that men do not fit in or are not being welcomed as nurses is a recurring theme within the literature. With gender bias firmly established as existing within nursing education, it seems apparent that the role of faculty in maintaining dominant femininities within nursing culture is a phenomenon that needs to be further explored.

\section{Tokenism and Group Dynamics in Nursing Culture}

The role of women in setting the expectations, norms, and behaviors for the nursing profession is commendable especially given that much of what nursing has become was created at a time when women had few rights and struggled against gender discrimination, lack of access to education, and societal expectations not to work outside of the home (Maggs, 1996; Poliafico, 1998). However, the time and place for using gender differences to predict the ability of individuals to succeed within a given profession is past. It is no longer socially acceptable for women to be held back or prohibited from professions that have historically been male-dominated. Similarly, it is now time for female-dominated professions like nursing to evaluate the anti-masculine gender bias and dominant femininities prevalent in their cultures in order to make changes that will usher in a more inclusive, more gender-balanced nursing workforce. The evolution into a more gender-neutral space necessitates a hard and objective consideration of current nursing culture, which might be characterized by Kanter's “theory of tokenism” (1977, p 206). 
The theory of tokenism is an attempt to understand how an individual's identifying characteristics such as sex, race, ethnicity, age, or religion, impact his or her social and professional advancement when he or she is a minority within the group (Kanter, 1977). Kanter explored the effects of how group proportions affect "interactions in groups composed of people of different cultural categories or statuses” (p. 965). Through this lens, Kanter (1977) looked at how group dynamics impact the members of a gender minority within the work setting and established a framework for “conceptualizing the processes that occur between dominants and tokens” within these groups (p. 965).

In order to help describe the situations that individuals might find themselves in, Kanter used the term skewed groups to describe when "there is as large preponderance of one type over another” in any social setting (1977, p. 965). Kanter named the minorities within skewed groups “tokens” and indicated that tokens have characteristics like sex, race, and age, which create a set of "assumptions about culture, status, and behavior highly salient for majority category members” and which have little to do with the ability of the token to do the work (1977, p. 698).

Kanter’s (1977) theoretical framework not only focused on the quantity of majority versus minority members within a group but also identified three different perceptions (visibility, polarization, and assimilation) that "determine the interaction dynamics between tokens and dominants and create the pressures dominants impose on tokens” (p. 971). With the visibility perception, Kanter noted that tokens are more visible within skewed group situations and that this increased visibility causes the dominants 
within the group to have more awareness of the token within the working environment. The polarization perception is defined by Kanter (1977) as the increased awareness of tokens by dominants within the group, which causes dominants to be more aware of the differences of the token within the group and will isolate or exclude the token from social interactions that may influence the token's professional mobility and success. Finally, with the assimilation perception, Kanter observed that dominants within skewed groups tend to generalize the most visible characteristics of the token to all members of the token group creating generalizations or stereotypes that are then attributed to all members of the token group (Kanter, 1977). These perceptions by the dominant group lead tokens to be impacted by the cultural norms and expectations of the dominant group and have little ability to affect change or impact the dominant culture in a way that is more inclusive towards them (Kanter, 1977). By connecting perceptual factors with the interactions between majority and minority members within skewed group settings, Kanter created a process for evaluating the quality of social engagement within dynamic social settings and for showing how the quality of social interaction among different groups impacts professional mobility (1977).

Kanter (1977) also applied her theoretical framework in a study focused on the effects of group dynamics of skewed groups where women were the gender minority in an industrial sales corporation. She found that "tokens appear to operate under a number of handicaps in work settings” and that performance pressures including stereotyping, social isolation, and the increased visibility of mistakes impact tokens working in skewed group settings. She concluded that the "investigation of the effects of proportions on 
group life and social interaction appears to be fruitful both for social psychological theory and for understanding male-female interaction” (p. 988), and she specified that her framework was "applicable to other kinds of tokens who face similar interaction contexts” (p. 985), such as men in nursing. To illustrate her point, Kanter cited a study by Segal (1962) of the experience of male nurses. Segal explored the idea of "status tensions in the nursing situation that are created or exacerbated when the nurses are males” (p. 31). Segal found that "the combined effects of three related factors, being different from female colleagues, being cut off from [male] physicians, and cutting themselves off from aides, tended to make male nurses isolated in the hospital social structure” (p. 2003).

Although Segal's study came before Kanter developed her theory, this early study of men in nursing highlights some characteristics that are consistent with Kanter's framework. Specifically, the men in Segal's (1962) study reported that they felt more visible as a gender minority within nursing, felt isolated by social restrictions related to being a gender minority, and had to justify their place in a role where they were not obviously perceived to fit. By mentioning men in nursing as a group that might experience tokenism, Kanter (1977) identified an area of research ripe for future exploration.

In the years since Kanter's (1977) publication, a few scholars have explored Kanter's ideas about tokenism for males in nursing, including Fairhurst and Snavely (1983), who used a quantitative methodology to ask if males experience tokenism as members of the minority group in nursing. A questionnaire was distributed to 41 males and 281 females in nursing school, and both genders were asked to respond to questions 
related to social engagement and isolation. Interestingly, the results of this study did not find a statistically significant result indicating that male tokens were more socially isolated than their female counterparts, which led the researchers to conclude that men do not experience tokenism in nursing (Fairhurst \& Snavely, 1983). However, the Fairhurst and Snavely (1983a) sociometric questionnaire did not appear to specifically ask the participants to rate their feelings of isolation or experiences of loneliness, and it did not address questions related to visibility, polarization, or assimilation, which Kanter (1977) specifically discussed as areas of tokenism; this could have impacted the results.

In another study of male nursing students, Heikes (1991) re-examined the issue of tokenism in male nurses with a sample of 15 men who were employed in a hospital setting. Heikes found that the male participants felt more visible within the organization and that they encountered informal social isolation related to how often they were prescribed attributes like ladder climber, troublemaker, He-man, or stereotyped as gay based on their gender. Heikes noted that males may not experience tokenism in the same way as women in similar situations. However, “the male nurses interviewed experienced heightened visibility and responded to the resulting performance pressure by overachieving” (Heikes, 1991, p. 396).

While these two studies focused on men in nursing school, Floge and Merrill (1986) used a qualitative study to examine the effect of tokenism of male nurses and female physicians in two hospital settings. With over 540 observation hours and corroborating interviews with minority and majority members of the hospital staff, Floge and Merrill (1986) identified that male nurses did experience the effects of tokenism in 
the work place. Specifically, their research showed that the male nurses were more visible to patients and colleagues; that they were excluded from conversations by their female peers as well as from topics related to families, babies, and dating, or alternately the men excluded themselves from these conversations; and that the male nurses tended to socialize with male physicians instead of their female colleagues (Floge \& Merrill, 1986).

In addition to these research findings, that study also found evidence to support Kanter’s (1977) concept of “status leveling” (p. 980). “Status leveling involves making adjustments in perception of the token's professional role to fit the expected position of the token’s category” (Kanter, 1977, p. 981). Kanter expanded on this by giving the example of women in her study who were treated as secretaries by the men in the workplace although their professional status was equal to the men's. In this example, the gender of the women superseded the status of their professional role and became the dominant characteristic with which the men engaged with the women socially. In the case of men in nursing, status leveling has the potential to advantage the token male because they get ascribed the dominant characteristics of their gender, which may be viewed as a higher status instead of the status identified with their professional role (Floge \& Merrill, 1986: Kanter, 1977). In their study, Floge and Merrill (1986) found that some men in nursing benefited from their token status in the workplace, were granted the benefits of their male status, and tended to be viewed with "characteristics such as authority, greater competence, and leadership which should favorably influence their careers” (p. 942). 
This idea that some male tokens may benefit due to having gender or race characteristics that trump their professional role status was also explored by Wingfield (2009). Through the use of semi-structured interviews with 17 male Black nurses, Wingfield (2009) explored how race and gender interplay with token status for men in nursing and used the metaphor of the "glass escalator" to explore how an individual's dominant characteristic allowed some tokens to advance farther than those within the majority group or other tokens with lower status. Wingfield (2009) found that for black male nurses, the characteristic of race became the dominant feature for the token and "that the upward mobility implied by the glass escalator is not uniformly available to all men who do 'women's work” (p. 5). Additional studies looking at the implications of race and gender for tokens in skewed groups in the field of law enforcement have also supported Kanter’s (1977) theory and have found that tokens experience increased visibility, isolation, performance pressure, and barriers for upward mobility (Gustafson, 2008; Stroshine \& Brandl, 2011).

Although there is disagreement in the literature about the level of tokenism males experience within the nursing culture, it is clear that some gender dynamics are involved when males join the profession. By applying Kanter’s (1977) theory of tokenism to the barriers identified in the literature for men in nursing, we have identified a lens to help explain why men are experiencing social isolation, gender stereotypes, and role strain within nursing.

\section{Summary}


The experience of men in nursing has been evaluated by qualitative and quantitative researchers, with social isolation, gender bias, and role strain found to be persistent barriers for male nurses in both the education and workforce settings (Bartfay et al., 2010; Bell-Scriber, 2008; Fairhurst \& Snavely, 1983; Holyaoke, 2000; Kelly et al., 1996; Snavely, 1983). Causal factors for these barriers for men within the nursing environment point to the dominant femininities of the nursing profession and the differences in how men engage with patients and express the core concepts of nursing education from a male perspective (Brady \& Sherrod, 2003; Grady et al., 2006; Shelton, 2003, 2012; Williams, 2010). Kanter's (1977) theoretical framework of how numerical minorities are perceived by the dominant group in the work setting is helpful for understanding the barriers that men experience within the nursing profession as tokens within a predominantly female profession. However, there are a number of gaps within the literature that need to be explored.

While the barriers of social isolation, gender bias, and role strain have been identified for men in nursing, the link between these experiences and the effect that they have on male graduation and retention rates within nursing education are currently unexplored. Understanding how these barriers impact male nursing student success in the educational environment is important for creating a learning environment that is welcoming to men within nursing education. In addition, some researchers have found that men may experience professional benefits as minorities within the nursing workforce (Fairhurst \& Snavely, 1983b). However, there is no current research on whether men receive benefits within the academic environment that help them succeed. Identifying 
what factors impede or increase male nursing student success in the educational environment is critical for addressing inequities within nursing education for all minority groups.

Another gap in the literature exists regarding the role of faculty as enforcers of the dominant femininities within nursing culture and their understanding of the barriers that men face while in nursing school. With a growing body of literature identifying the gender bias and discrimination found within nursing education, the role of faculty in maintaining or challenging these existing issues is unknown. While researchers have made recommendations to nursing educators to help address and alleviate the barriers that have been identified, only a few researchers have begun to assess the awareness of these gender issues by nursing faculty. In fact, research is just now beginning to explore the additional aspect of how faculty interaction may play a role in the student experience for male nursing students (Bell-Scriber, 2008; Meadus \& Twomey, 2011). However, these researchers have not assessed the awareness that nursing faculty may have about the barriers that men may experience within nursing education. In their role, faculty act as enforcers of existing nursing culture for all students, which may contribute to the barriers that men experience within nursing education. Understanding the dynamic between faculty members and male nursing students is central to identifying and removing barriers in the learning environment and ultimately changing the educational climate for all students. For these reasons, the role of nursing faculty as the gatekeepers to the knowledge, skills, and cultural norms within nursing education needs to be examined in relation to the barriers that men in nursing experience within the educational setting. 
Finally, while a few researchers have tested Kanter’s (1977) theory of tokenism with men in the nursing workforce (Fairhurst \& Snavely, 1983b; Floge \& Merrill, 1986; Heikes, 1991), this framework has not yet been applied to male nursing students within the educational setting. Kanter (1977) observed that "numbers do appear to be important in shaping outcomes for disadvantaged individuals” (p. 988), and although Kanter (1977) originally posited her theory within the work setting, the prevailing feature of her theoretical framework is that the proportion of minority to majority members within a group setting is what dictates the effects of tokenism within a skewed group. This is true for men within nursing education as well. The number of men within nursing education makes them a minority and the literature has identified that they experience a number of barriers (Anthony, 2006; Denhart, 2008; Meadus, 2000; O'Lynn, 2004; Roth \& Coleman, 2008) that are similar to the effects experienced by tokens in skewed groups in work environments (Kanter, 1977). By applying Kanter's theoretical framework to the educational setting, we can assess its efficacy in settings beyond the workforce. In addition, Kanter’s framework was proposed to understand the social factors within the work setting that influence upward mobility for tokens within a specific work environment. By applying that framework to the educational setting, we have a lens for assessing the role of faculty in influencing male nursing student success within the classroom.

There are a number of issues left unexplored on this topic within the literature at this time, including the effect of isolation, gender bias, and role strain on student success; the awareness of faculty about gender differences within nursing education and the use of 
Kanter's framework as applied to an educational setting where numerical minorities exist.

Given that the number of men in nursing education will not dramatically increase overnight, there is a need to find ways to lessen the barriers that male nursing students experience while in nursing school. It is critical to address the barriers that men face within nursing education in order to support the learning needs of the growing number of men joining nursing programs today, to remove discrimination and stereotypes from the learning environment overall, and to support the learning and skill building of all students. 


\section{CHAPTER III: RESEARCH METHODOLOGY}

As the body of literature highlighting the differential experience for men in nursing has grown, so too have the implications for nursing education. Meadus and Twomey (2011) called for nursing education to "be more proactive in promoting an appropriate learning environment for men students," noting that "nurse educators need to re-evaluate their teaching strategies and develop greater awareness of possible biases toward men in nursing to provide a gender-neutral environment for all students” (p. 277). Stott (2006) also stated that "nurse educators need to be cognizant of gender differences in learning styles and foster a learning environment that focuses on independence as a means of promoting competency and achievement” (p. 331), and Streubert (1994) remarked that "nurse educators need to develop learning situations that support [male] functioning in a female-dominated profession” (p. 32).

Researchers have also identified specific ideas for nurse educators to help bridge the gender gap within nursing education. Recommendations from the literature to alleviate gender bias in nursing education include the creation of mentorship and retention programs, increased faculty awareness of gender bias and the use of genderneutral language, and the recommendation that nursing programs should adapt in order to be more inclusive of men in nursing (Alfred, et al., 2011; Dyck et al., 2009; Roth \& Coleman, 2008; Smith, 2006; Stickney, 2008;). Although these calls to action have been increasing from a growing body of investigations, very little evidence indicates that nursing faculty and administrators are aware of these gender issues in the classroom. 
With faculty playing a critical role in the socialization and success of male nursing students into the nursing profession, it is critical to understand what faculty know about the issue of men in nursing and to identify to what extent, if any, faculty are adjusting their instructional practices and expectations in order to meet the perceived needs of the male students in their classrooms.

This study explored the learning environment for male nursing students and examined how men interacted in this environment. Specifically this study investigated the nature of the interactions between nursing faculty and male undergraduate students in the educational setting and how gender is described, represented, and reflected within the classroom setting. Finally this study used Kanter's (1977) theoretical framework of tokenism to identify if male nursing students were perceived differently by faculty members while in the classroom and if so, what affect, if any, did this have on male nursing student educational success.

\section{Qualitative Research Strategy}

This study explored the complex relationships and experiences of specific students and faculty members within an identified educational context and described the experience, feelings, and perceptions of the participants from this shared educational experience. Given the context of where this phenomenon occurs, the research methodology chosen for this study allowed for the exploration of student and faculty interaction within the natural educational setting and sought to further highlight the linkages between male nursing students, their faculty, and their educational environment. An initial review of quantitative and qualitative research methods identified that a 
qualitative research method that "follows the exploratory scientific method" and is designed to “describe what is seen locally” (Johnson \& Christensen, 2012, p. 33) confirmed that a qualitative method would best meet the exploratory and descriptive needs of this study.

The merits of qualitative research are discussed by a number of authors. For example, Johnson and Christensen (2012) observed that "qualitative research is used when little is known about a topic or phenomenon and when one wants to discover or learn more about it” (p. 33). Other researchers noted that "qualitative researchers study things in their natural settings, attempting to make sense of, or interpret, phenomena in terms of the meanings people bring to them” (Denzin \& Lincoln, 2005, p. 3). Finally, Creswell (2007) indicated that qualitative research "includes the voices of participants, the reflexivity of the researcher, and a complex description and interpretation of the problem, and it extends the literature or signals a call for action” (p. 37).

Qualitative research methods may come under scrutiny for using a broad lens where human behavior is viewed as "situational, social, contextual, personal, and unpredictable” (Johnson \& Christensen, 2012, p. 34) instead of “regular or predictable” (Johnson \& Christensen, 2012, p. 34). However, it is clear that a qualitative approach is appropriate for this study because it allowed this researcher to view the nuances contained within a nursing program context between nursing faculty and students in a Pacific Northwestern medical university (PNMU). 


\section{Study Design}

Of the qualitative approaches available, the case study was the best fit for exploring these research questions because the experiences of men in nursing school are directly connected to their interaction with their female peers, faculty members and administrators in a context that needs detailed exploration and description (Creswell, 2007). Yin (2009) noted that a case study should be used when the "research question is in the form of a how or why," when the study does not require the "control of behavioral events," and when the study "focuses on contemporary events" (p. 8). This case study research meets these criteria as the phenomenon being explored is how faculty and male nursing students interact in the classroom and the research does not require controls within the setting that would limit or restrict the natural activities of the participants. In addition, Yin (2009) stated that "the case study is used in many situations to contribute to our knowledge of individual, group, organizational, social, political, and related phenomena” (p. 4). Given that little is known about how the interaction between faculty and male nursing students can impact the perceptions of gender bias in the classroom and future student success, this study seeks to better understand and explain the experience within the educational setting.

For this study, a single instrumental, within-site case study (Stake, 1995) was used to investigate the interactions between male nursing students, nursing faculty, and administrators during the students’ first term of nursing school. In this method the "researcher focuses on an issue or concern, and then selects one bounded case to illustrate this issue” (Creswell, 2007). The single instrumental case study design (Stake, 1995) was 
well suited for this subject as the interactions between female nursing faculty and male nursing students are currently unexplored, and investigating this topic sought to uncover a deeper connection between the perceived biases that male nursing students experience within the educational environment. Yin (2009) noted that it is appropriate to use the single case study method when "the objective is to capture the circumstances and conditions of an everyday or commonplace situation” (p. 48) and Creswell (2007) stated that the case study approach allows for "in-depth data collection involving multiple sources of information” (p. 73).

The boundaries for this case study were established as the first nursing class that all nursing students take during their first term of the undergraduate nursing program at the PNMU. At this particular institution, all students in the undergraduate nursing program complete the same series of nursing courses in a lock-step manner, and all new nursing students take a course called Nursing Foundations (also known as Nursing 210 $\mathrm{A} \& \mathrm{~B})$. The course catalog describes this class as an introduction to the curriculum where students use reflective thinking about their practice, and it includes classroom and clinical experiences (PNMU Course Catalog and Student Handbook, 2012). Nursing 210 A\&B is a nine credit class wherein students spend four hours each week in class reviewing nursing history, theory, and learning the fundamentals of health promotion. The students have an additional four hours each week of clinical coursework applying core concepts in practice. Given that this is the first course offered in the nursing curriculum for all undergraduate nursing students, it can be seen as a primary way for new nursing students to begin their immersion into the nursing curriculum and culture. In addition, all of the 
faculty involved with teaching Nursing 210 in the fall of 2013 (the period of study) were women. Thus, new nursing students did not encounter a male nursing faculty member as part of their initial educational experience at the institution during this term. This setting was a prime location to explore the gender dynamics between nursing faculty and male nursing students in an undergraduate program and provided the boundaries for the case.

\section{Sampling}

The boundaries for this case study were designed around the educational environment for male nursing students during the fall term 2013 Nursing 210 A\&B course. All the students and faculty associated with this course were included in the phenomenon under study and were included through purposeful sampling (Patton, 1990). The sample size was limited to the specific number of students enrolled in the course and the number of faculty assigned to teach the course. This course had 40 new nursing students, of which two were men and the rest were women. Thus, the men made up 5\% of the class. Although this was a smaller sample than anticipated for this course, overall it is reflective of the national average of men in nursing at 6.8\% (U.S. Department of Health and Human Services, Health Resources and Services Administration, 2008). Four women declined to participate in the study. All other students and faculty members participating in the study were women, which is similar to the national statistics for women in nursing. After informed consent was obtained, both men and 34 women participated in the study for a total of 36 student participants. The course had two lead faculty members, one who taught the theory coursework in Nursing 210 A and one who lead the clinical coursework in Nursing 210 B. A third faculty member taught sections of 
both the theory and clinical classes. These three faculty members along with the program director of the undergraduate curriculum made up the faculty participants.

\section{Methods}

The research procedures for this case included in-class observations; student, faculty, and administrator interviews; and instructional and curricular material document analysis. "The use of multiple sources of evidence in case studies allows an investigator to address a broader range of historical and behavioral issues" (Yin, 2009, p. 115). Collecting data from multiple sources increases the opportunity to find evidence supporting or contradicting the research question. When similar evidence across sources is found, the findings are strengthened because the evidence is supported by more than one perspective. “A major strength of case study data collection is the opportunity to use many difference sources of evidence” (Yin, 2009, p. 114). In addition, collecting data through the use of multiple sources is an important part of corroborating the findings between sources. Identifying multiple sources that relate to key findings increases the reliability of the case study research and increases the opportunities for triangulation (Yin, 2009).

The study was set to begin at the start of fall term 2013; however, the project needed institutional review board (IRB) approval at both the institution where this researcher is a doctoral student and also at the institution where the study was occurring. This additional level of IRB approval took longer than anticipated, and IRB approval from the second institution did not come through until the fall term had already begun. This delay pushed back the start of the data collection by three weeks. This meant that the 
timeline for gaining access to the participants, garnering informed consent, and starting data collection began in the third week of the term.

\section{Access to the Site}

As an administrator at the nursing school where this study was conducted, this researcher already had established professional relationships with one of the faculty teaching the fall theory section of the Nursing 210 course as well as with the program director overseeing this program. Each section of the course is taught by a different faculty member and is located on separate sites. The faculty and program director for the course helped provide this researcher with key information and access about the course, such as dates and times the classes would be held in the upcoming term as well as the names and contact information of the other faculty involved with the course. The connection with these two individuals at the site helped introduce the researcher to the other faculty members and students associated with the course. The endorsement of these key informants helped give credibility to this project and provided the researcher with direct access to the students in the classroom. Once IRB approval from both institutions was granted, the researcher notified these key informants about the project and gained access to the site in order to begin participant recruitment. 


\section{Participant Recruitment}

The major part of the nursing coursework takes place on the main campus or in the immediate urban area of a Pacific Northwestern Medical University. In order to gain access to this classroom environment, the researcher recruited the lead faculty members teaching Nursing 210 A\&B to participate in this study and asked to be allowed to observe the class on pre-arranged dates. The recruitment of the faculty members occurred through direct conversations and through email invitations. Students were recruited through an inclass presentation by the researcher covering the purpose of the study and a review of informed consent. All students were also sent a follow-up email. Consent was gained from all but four female nursing students. The students who did not provide consent were omitted from the researcher's observations and the in-class study materials created by these four students were not collected by this researcher or used in any way with this study. Pseudonyms have replaced all participant names.

\section{Participants}

The participants in this study consisted of 36 new nursing students enrolled in Nursing 210 A\&B, three faculty members associated with that course, and the program director who oversees the undergraduate nursing curriculum for that campus. Two of the student participants were male, age 26 and 32. Both identified as white and joined the nursing program as a second career. The remaining 34 female students had an age range of 22 to 52. Two of the women identified as Native American, five identified as Asian, and four identified as Hispanic or Latina. All but one of the students were Oregon residents at the time of application to the program. Ten of the students indicated that they 
came from a rural background, and 13 of the students indicated that they came from a socioeconomically disadvantaged background. The faculty members and administrator participants were all white women with an age range between 35 and 50, all had over 10 years of experience in nursing, and three of the four had over 10 years of experience teaching nursing.

\section{Informed Consent}

The researcher was a doctoral student in the EdD program in Higher Education and Leadership at Portland State University and was also an administrator at the Pacific Northwestern University where the study occurred. This study was reviewed and approved by the institutional review boards at Portland State University and at the PNMU. All faculty, students, and administrator participants in this study were over the age of 18. This researcher recruited participants and reviewed informed consent with all participants before engaging in observations or interviews. Informed consent applied to four different groups: faculty members teaching the course, male nursing students in the course, female nursing students in the course, and the undergraduate program director connected to the course. Informed consent forms, found in the appendix, were reviewed and obtained for all participants by the third week of the term.

Faculty granted access to the Nursing 210 A\&B class for observation of the class on agreed upon days and allowed for the participation in an in-class activity. Program director consent allowed for interviews at the end of the term and for those interviews to be electronically recorded for further analysis. Consent from female students allowed for observation of the Nursing 210 A\&B class and for participation in the in-class research 
activities. Consent from the male students allowed for observation in the Nursing 210 A\&B class, allowed for review of the in-class research activities, and provided permission for the researcher to interview the students and for those interviews to be electronically recorded for further analysis.

The informed consent form described the activities and methods associated with the study and the potential risks and benefits from participating in the study. The informed consent form also identified that participants had the right to withdraw from participating in the study at any time. The researcher assured participants that evidence of every participant's informed consent would be retained for three years after the study was completed.

\section{Observations}

After informed consent from all participants was obtained, in-class observations occurred during the third, fifth, seventh, and tenth weeks of the fall term. Observations took place in both theory and clinical coursework and ranged from two- to four-hour intervals. During the observations, the researcher noted student-to-student interactions, faculty-student interactions, student participation in class, and course content. This researcher took field notes during in-class observations which are described by Marshall \& Rossman (2011) as “detailed, nonjudgmental (as much as possible), concrete descriptions of what has been observed” (p. 139), and spent time reflecting and journaling about the experience after each session. 


\section{Interviews}

The researcher conducted face-to-face interviews with selected participants including both male nursing students, three course instructors, and the program director responsible for the curriculum. “Combined with observation (looking, hearing, smelling, or touching), interviews allow the researcher to understand the meanings that everyday activities hold for people” (Marshall \& Rossman, 2011, p. 145). A focused interview format was chosen to best elicit the "subjective experiences of persons exposed to the pre-analyzed situation in an effort to ascertain their definitions of the situation” (Merton, Fiske, \& Kendall, 1990, p. 3). The faculty and administrator interviews occurred once at the end of the term. The researcher conducted a total of eight interviews, and all participants who were asked to interview participated. Most of the interviews lasted about an hour although however one faculty interview and one student interview went as long as two hours.

Interview questions were developed specifically for each individual based on his or her role as a student, faculty, or administrator. Interviewees within the same group were asked the same set of questions, and the mid-term set of questions differed from the end-of-term questions for the male nursing students, who were interviewed twice. The male nursing students were asked questions related to their identity as a male nursing student, about their connections and comfort level with other students and faculty in the class, about their academic success in the class, and their overall experiences as a male in the program. Nursing faculty and the program director were asked about the expected educational goals for Nursing $210 \mathrm{~A} \& \mathrm{~B}$, the socialization of new nursing students to the 
field of nursing, and their knowledge of gender differences for male nursing students in the classroom and within the nursing profession overall. They were also asked about their connections with students in the program, about any academic differences they noticed between male and female nursing students, and their overall impressions of the openness of the profession towards male nursing students. The program director was also asked additional questions related to whether gender awareness was addressed with the faculty in a broader educational context, or if issues that men appear to experience in nursing had ever been addressed as a training or awareness campaign for the faculty. A complete set of interview questions is found in Appendix D.

All interviews were digitally recorded and the researcher took notes to guide the conversation; however, the questions were structured to elicit a broad range of responses from the participants and the researcher did not restrict the responses to particular topics or impose time constraints (Merton et al., 1990). The interviewees were asked to illustrate their points by providing examples or more description as they went along. Each interview was transcribed for thematic analysis.

\section{Document Analysis}

Along with in-class observations and participant interviews, the researcher reviewed course instructional materials including the course syllabi, teaching materials, course descriptions, and course outcomes. In addition, an in-class drawing activity was administered at the fourth and eleventh weeks of the term. Students were given 15 minutes before the end of the class period and were provided with crayons and a blank sheet of printer paper. They were then instructed to "draw a picture that describes your 
educational experience as a nursing student so far.” The same materials and instructions were provided both at the mid-term and at the end of the term. These drawings were then analyzed for themes and incorporated into the review of the course materials.

\section{Dedoose $\odot$}

Dedoose ${ }^{\circledR}{ }^{\circledR}$ is a software program that allows for the storage and coding of qualitative data. This web based software is accessible from any computer with Internet access and allows for the uploading of documents that can then be stored, sifted, and coded for themes. The use of Dedoose $@$ served to bring all of the research data into one system and allowed for all data to be analyzed using unique identifiers so that participant confidentiality could be maintained at all times. The software also allowed the researcher to assign and track codes systematically across all data types, easily cross referencing coded data and quickly accessing data related to specific themes.

Yin (2009) indicated that data analysis software can "help you code and categorize large amounts of narrative text” (p. 128). All data collected from the study was converted into text format and uploaded into Dedoose. It is important to note that the Dedoose software is a qualitative tool that can organize the multiple pieces of evidence collected from a case study and then sort and organize the evidence based on the theoretical propositions related to the research study. The Dedoose software does not automatically apply the codes to the evidence, nor did this researcher rely on the software to draw conclusions about the data. Yin (2009) stressed that the use of software in the analysis process is just one tool that a researcher should use in the process and that any patterns identified in this method "will still be conceptually more primitive” (p. 128). 
Parent codes were used as part of the analysis of the material in Dedoose, and the researcher linked the initial propositions to these parent codes in order to get a larger picture of the overall evidence.

\section{Data Analysis}

The data collected for this study were analyzed together to develop themes from multiple sources (Yin, 2009). Data from these interviews, observations, and academic documents were reviewed for themes, typologies, and clusters (Marshall \& Rossman, 2011) by the researcher and then coded and tracked using Dedoose $\subset$. Coding decisions followed the "theoretical propositions" (Yin, 2009, p. 130) that led to studying this topic and information was included if it (a) captured the nature of the interactions between nursing faculty and male undergraduate students in the educational setting: (b) addressed how gender was described, represented, or reflected within the classroom setting: and (c) related to the perceptions of visibility, polarization, and assimilation as identified in Kanter's (1977) theoretical framework of tokenism.

The analysis began with reading the interview transcripts, class observations notes and academic material multiple times to get an overview of the material (Patton, 2002). Then each document was reviewed for any evidence connected to the initial concepts relating to the educational experience for men in nursing. These concepts found in the literature included the image of nursing, academic success, discrimination, and tokenism. Codes were also developed as patterns emerged from the analysis and new categories for feelings and clinical setting were created. The researcher used Dedoose $₫$ to tag codes as they occurred within the documents and the software captured the frequency of each code 
that was used as well as identified when codes intersected within the same set of text as part of a matrix analysis. Data was reviewed for accuracy and triangulated (Yin, 2009) for themes with this researcher's advisor as well as through conducting member checks with the participants to confirm accuracy of data and check researcher perceptions. By centering the data analysis on the core areas of faculty and student interactions, perceived gender bias in the classroom, and Kanter’s (1977) theoretical framework for how gender bias may influence student success, the researcher was able to "focus attention on certain data and to ignore other data” (Yin, 2009, p. 130), which assisted in identifying themes, patterns, and category names related to this research topic.

\section{Summary}

This case study explored the educational environment for men in nursing school and the faculty who are responsible for preparing them to join the nursing profession through the use of interviews, in-class observations, and document analysis of a fundamental nursing course that all nursing students at the site institution must complete in order to progress in the program. The data were analyzed through the lens of the study questions and care was taken to ensure that the analysis was credible and valid. 


\section{CHAPTER IV: FINDINGS}

This single instrumental case study (Stake, 1995) analyzed data from multiple sources related to a Nursing 210 A\&B course taken by first-year students enrolled in the PNMU nursing program. Data were collected in the form of direct in-class observations for both sections of the course, interviews with the male nursing students, interviews with faculty teaching in the course, an interview with the program director who oversees the undergraduate curriculum, and analysis of the documents associated with the course including the course catalog description, the course syllabi, and curricular materials that were present in class all supported by researcher memos. The evidence was then sorted and organized using the theoretical propositions analytic strategy proposed by Yin (2009).

Yin (2009) noted that beginning data analysis by choosing an analytical strategy will re-focus the researcher on the questions that inspired the case. For this study the original propositions were formed based on the following assumptions: 1) men are isolated from faculty and peers in nursing school; 2) men experience barriers in the educational environment; 3) as gender minorities, men may not be as academically successful in a female-dominated educational environment. The research protocols for this study were based upon these theoretical propositions, and the initial evidence was organized and sorted by codes that related to each of these theoretical propositions.

This chapter also describes what the researcher learned from interviews, observation, memos, and analysis of related documents. These descriptions are presented 
in order of the theoretical propositions that formed the basis of this study and interview protocols that guided this study. The focal point of the interactions in this study took place in the Nursing 210 A\&B course and the interviews and observations with the participants provide the best way to describe the educational environment for male nursing students and are used to help describe the phenomenon under discussion.

\section{Theoretical Proposition 1: Men Are Isolated from Faculty and Peers in Nursing}

A fundamental assumption of this study is that men may be isolated from faculty and female classmates in the educational environment of the PMNU nursing program. Additionally, this researcher took from the literature that the quality of interactions with faculty and female classmates are shaped by gender. This section reviews the data in light of the interactions that the men had with each other, their female peers, as well as with their faculty. In addition, this section highlights the faculty perspective of the men in the nursing program.

\section{Male Nursing Students: Interactions with Each Other}

During the interviews, both men discussed the importance of having another male in the program to bond with and the ease with which they immediately connected. Cliff talked about the importance of bonding with another male student in his first interview:

I definitely felt lonely at first and I think that was because, I'm trying to think how to describe it. Well, I didn't really know anybody in my group and there were so many women in... in the group that I kinda wanted to bond with a male. And there weren't so many around, you know, especially at the orientation. I remember there was one male that came up and introduced himself and we talked for a little bit and he wasn't in my program. And I just remember wanting to find, like who is the guy in my group so we can like be buddies and I can have 
somebody to hang out with? I don’t know why that's so important but it just felt important, you know.

Like Cliff, Jack expressed an interest in finding another male to connect with at the beginning of the term, indicating that when he saw Cliff for the first time, "I gave him a high five and...in...in orientation and he's like, you know, instant best friends. It's kind of just like, it is what it is. I would feel like a single minority out of 39 other women if I was by myself.” He went on to say, "If I was the only male, it would have been much more evident. But it’s nice to have him there.”

In-class observations showed that that Cliff and Jack consistently engaged with each other and shared a good rapport. They sat together almost exclusively in each class throughout the term and were seen to turn to each other to clarify questions that came up in class. Additionally, they typically worked together in small-group activities, and they often were seen speaking with each other before and after class in a social and friendly manner.

\section{Male Nursing Students: Interactions with Female Classmates}

While Cliff and Jack seemed to make a connection with each other almost immediately, establishing connections with their female peers in the class appeared to take longer for both. For example, Cliff described his first weeks in the class as having been good overall but he also shared that making connections with the other students in the class had been confusing.

I feel like there's been times that I feel like I'm getting a connection with someone um, digitally and then in class I feel like there's a disconnect and I don't understand that. I'm kinda like, it seems weird this person wants to talk to me 
outside of school via email/text message or whatever but in class it's almost like they avoid me or something, you know.

Cliff also talked about the cliques that seemed to form in the class and from which he was excluded.

A lot of certain groups are hanging out together, which makes sense. It's like I feel like that's what we're all doing, you go to what's comfortable first and then you kinda start branching out. Jack and I definitely stuck together first and we've kinda branched outwards as we've met more friends.

He went on to describe a situation during lunch when a group of female students sat next to him at the lunch table but then proceeded to exclude him from the discussion choosing to chat among themselves:

I'm just eating my sandwich like man, I don't belong here. I don't know what's going on. I've nothing to contribute and I just felt awkward because I felt like they didn't know I was sitting there and I just sat there and they're like why is he here? That was kind of how I felt.

He said “I don’t think they even acknowledged that I was there, but I definitely didn’t feel welcomed and I didn’t feel like I could contribute. Oh yeah I’ve experienced that.” A change occurred as the class progressed however, and in his second interview at the end of the term, Cliff reflected on this interaction with female classmates again. He indicated that by the end of the term the situation had resolved itself:

One of the women I was sitting next to [during that lunch example], we ended up talking out in the community. She sat next to me and we just started chatting and I was like cool. I thought it was, I felt like such an outsider at that point in time and then she included me and I told her that when we met up for margaritas, I said that really meant a lot to me that you came and sat next to me that one day we were at the school. I told her the story about... and she's like oh, that's so sweet. I was like I'm just kind of sensitive because it was like a new situation for me in like, it's just kind of funny now how I felt like that, and now I don't. 
This sense that Cliff became closer to his female classmates and had better connections

with them as the term went on was evident in his second interview:

Yes, I think that I feel a lot closer to my cohort in general. I think it took some time to kind of get a chance to be in a scenario with everyone because in the theory classes, you don't really get an opportunity to mix and talk. I mean people tend to sit with their friends that they know and then you can't really talk in class so I wouldn't want to be talking to someone in class so that's kind of out but I think that the points where we went out to the community and has some group projects and stuff really gave us a great opportunity to mix people up that wouldn't normally have the chance to talk. In clinical it's definitely, I think, the better opportunity to know someone because you get to do all these activities and practice your skills and you get to work with so many...get to have some small chats.

Cliff showed an interest in making friends with all of the students in the class and seemed to start off the term feeling like a self-conscious outsider. By the end of the quarter though, he seemed to have made the connections with the students that he was seeking and seemed more comfortable with his peers. For Cliff, the in-class group work and excursions during clinical outings seemed to foster interpersonal collegiality.

From the beginning, Jack seemed to be less concerned with making friends with all members of the class and talked about a few specific connections that he had within the class. These connections included Cliff as well as a few other female peers. When asked about making connections with women, Jack stated, “Um, but being able to interact with the women, how that has been? Pretty...pretty seamless I guess.” Jack went on to describe meeting another student in the class and establishing a friendship with her early on:

Holly, I saw her at, Wal-Mart...because I was getting supplies and the cashier asked me, where are you going to school and I was like, nursing school at PNMU and Holly was like right next to me in the same checkout stand. And she's like I'm going to PNMU too. What term? You know what...we found out we were in 
the exact same cohort. And then we found out that she was, um, in some anatomy physiology classes with my fiancé. So... and she knew her so then, it was like instant bond there she is. Um, and we got each other's phone numbers and she's been a really cool person the whole time, so you know...-

Jack also mentioned that he noticed specific groups that formed in the classroom.

I think that's what we gravitate towards in the beginning; trying to find those that are similar to you until you get to know people. I think over time the cliques are probably gonna be more overlapping. In the beginning [cliques] are really impactful [because] those are the friends you make, those are the friends you plan to stick with, and these are the study groups you're probably gonna have the whole time.

Jack also talked about the support that his peers provided towards his academic success:

“I’ve realized I need a lot of student support, you know. We need to work together.” Jack felt that it was pretty easy to make friends in the program: "I guess you noticed that when you are not the only one texting everybody else, people are texting you for help too [which makes me think], so okay I'm needed as well.” Jack appeared less concerned with making friends with everyone in the class and established a few close connections early on. These early connections may have served as a foundation for him to forge relationships with other female classmates with greater ease.

Although social connections seemed to be formed during the course of the term with their female peers, both Cliff and Jack noted situations in the clinical setting where partnering with women made them uncomfortable. In addition, both men expressed worry about making the female student uncomfortable by having to practice clinical skills with them. Cliff stated: “it’s more like uncomfortableness when we do skills on each other. I just dread getting paired up with someone who doesn't want me to work with them.” He expressed that these feelings of discomfort went both ways; the women 
seemed just as hesitant to pick Cliff or Jack as a partner in class exercises. Cliff explained, “I guess I kinda feel uncomfortable sometimes working with the female students. It's not necessarily me that feels uncomfortable. I feel uncomfortable for them to have to work with me.” This is further illustrated by his comments that "it's more uncomfortable for me when I am doing the head-to-toe exam on a fellow female student because you have to do the heart [touch the chest area over the heart] and you don't want to touch anything. And there's really no way to do what you need to do without touching their chest area. I feel uncomfortable for them.”

Cliff suggested that the discomfort with doing clinical skills on another student has to do with the difference in the type of relationship that established between peers instead of between practitioner and patient. He said, "I think having it be another student makes it more different because you know this person a little more. You know because you have the more acquaintance type relationship you are more sensitive to stuff than a normal patient who you may not see again or you're not going to see that often.”

In a similar vein, Jack mentioned this discomfort with partnering with female peers in clinical activities. “I wasn’t uncomfortable, working with a female. I am pretty comfortable in myself and I have a fiancé [and] I'm a medic and things are professional for me, but I just didn’t want to make any of the girls uncomfortable.” However, Jack shared an experience in which a female classmate turned him down when the students were partnering with each other to practice foot exams. He said, "I think Bell was still uncomfortable about her toes being examined by me. So she’s like ‘oh, I have Piper [a female classmate] and we are going through the practice exercise.”” He stated that "with 
head-to-toe assessment, I picked Cliff just to keep it easy for all of the females that may be uncomfortable.”

Direct observations of both theory and clinical class situations showed that Cliff was more interactive and engaged with those around him than Jack. While Cliff was often observed inquiring about others and having short conversations or interactions with the other students, Jack was often observed as being quiet, making minimal eye contact with others, and engaging himself by looking at his text books, computer, or cell phone. The researcher observed that the women often sat with each other in both classes and engaged mostly with each other, walking in together, sharing personal stories, and working collaboratively on class activities. Cliff, Jack, and some of the women did form mixed groups for small-group work in the theory classroom especially when pre-matched by faculty in the clinical setting. In these scenarios the male and female students appeared to work well together. However, voluntary pairings between the men and women in the clinical setting were not noted, and this observer never saw mixed gender groups work on clinical skills together. The lack of male and females working together on clinical skills was also corroborated in a comment from faculty member Marcie in her interview. "I guess with thinking about health promotion sometimes men are a little uncomfortable with the physical assessment piece.” It seems as if at least one of the faculty recognized that clinical assessment can be uncomfortable for men.

\section{Male Nursing Students: Interactions with Faculty}

Feeling connected and supported. Both Cliff and Jack portrayed their faculty in positive terms and expressly described their interactions with faculty as establishing a 
"bond" or a "connection" with them. Cliff expressed his approval of the faculty overall stating, "I really like my faculty. I feel lucky because I feel that we have some of the most friendly and helpful faculty.” He shared a specific incident about his Nursing 210A instructor explaining, “with Susan, I knew that I would enjoy her class. I feel she and I are definitely bonding and we kinda joke with one another.”

Jack described his interactions with faculty at the mid-term interview in a somewhat less positive manner, but he indicated that he had not made a strong connection with his Nursing 210 A instructor. “I haven’t talked to her [Susan] personally in any office visits or anything, so it's been pretty superficial, but what she says is impactful in every class.” While Jack shared that Susan was a good teacher, he also offered this example of an important faculty connection that he made with another instructor.

Jillian is my clinical instructor for my clinical group and she's prior military [like Jack]. She was a nurse in the Army so we have this like instant bond, I think, so she tells me that she knows all these people who she can introduce me to and they have all these resources. As a result, Jack sees Jillian as a "really cool source of support so far.

It is clear that the men felt differing levels of connection with faculty and described their interactions with their faculty in a positive manner. This was highlighted when Jack stated, “The teachers are compassionate. They seem to really care about us, which is really cool.” In a similar vein, Cliff indicated, “I’m very happy about all the instructors, I feel like I have a good connection with all of them and my cohort.” 
Cliff and Jack's comments correspond with the observations of the researcher, who noted that the men appeared comfortable asking questions during class, volunteered answers when prompted by the instructor, smiled, laughed, and joked during break times with faculty, generally engaging in a positive, neutral way. This engagement is exemplified in an exchange when Susan was beginning to review the mid-term exam. Inclass observations found that the students were attentive and that Cliff asked about taking notes of the test questions. Susan jokingly engaged him, indicating that he was very "linear" and she was "not” showing an overall level of respect and comfort from learner to instructor. Generally both men were friendly with faculty and with both, faculty reciprocated. Finally, this seemed to be a positive conversation on both sides.

Themes from the evidence related to student interactions showed that both of the men they had a positive educational experience in their first term and developed relationships with each other, and with classmates and faculty. Some issues surfaced with initially making friends beyond each other, and both men shared the importance of having another male in the class to connect with at the beginning of the term. Cliff and Jack expressed positive relationships with their female faculty members and described their relationships as "bonded” or "connected.” This connection seemed to provide support for their academic experience and to help them feel comfortable describing their experience in positive terms.

\section{Faculty Perceptions of their Interactions with Students}

While it is informative to view the evidence around how the men interacted with each other, their female peers, and faculty within the PNMU nursing school educational 
environment, Yin (2009) believed it is also important to corroborate or triangulate what was learned from multiple viewpoints. This section reviews the faculty perceptions of their interactions and awareness of the men in the class to help understand a different perspective related to faculty-student interactions. The literature asserts that female nursing faculty may treat male nursing students differently than they treat the dominant population of female students. The data from faculty interviews is viewed with this lens to identify if the faculty and administrator in this class treat the men differently than their female peers.

\section{Nursing Faculty: Interactions with Male Nursing Students}

While the men described their interactions with faculty in largely positive terms, the faculty tended to describe their interactions with Cliff and Jack in terms of their personalities and their level of engagement in class. Nursing 210 A Instructor Susan noted that, “they are different 'types'; Cliff is pretty extroverted and intense, while Jack is very awake, alert and very present but not nearly as talkative as Cliff.” Polly, another instructor, noted similar personality types:

One is very vocal and the other not so much. Jack was hard to unpack. I couldn’t always tell how well he was doing because he didn't give a lot of eye contact in class and I thought to myself, is he dismissing this content? I would also think to myself that he might already know this because of his exposure in other aspects of healthcare. And so then I would try to ask a question later to sort of see how he was doing. By contrast, Cliff is like an open book being more forthcoming, which made it easier for me to know where he was at with something.

Marcie, the third instructor, also reflected on the personality characteristics of the students. : "One of them went with us to the schools and [Cliff] was very vocal, feeling really comfortable to speak up. In fact, I think he was one of two or three people who 
spoke most. Jack I don’t really know because he didn’t even talk much when I would go and talk to their group.”

While Marcie saw Jack as reticent to engage, Polly indicated that she saw Jack's comfort level in class grow over time. "My sense with Jack was that he maybe got more comfortable over time because once I saw what he was doing, I thought to myself that he is engaged and that's what mattered. It's when they look like they are not engaged [that I become concerned].” Susan, Marcie, and Polly noted that Jack’s personality trait of introversion contributed to their opinion of his ability to engage and presented more of an instructional challenge. They all agreed that Cliff was amiable and easier to teach.

Susan, Polly, and Marcie noted that both Cliff and Jack interacted closely in the class. Susan clarified this perception when she shared that "it's interesting though, not so much toward the end, but you and I talked about it, they were velcroed to each other. They sat next to each other every day. And I'm curious what that's about.”

Polly noted the same thing but felt that this was nothing unusual, explaining that this was typical behavior as students get to know each other and find common ground as first-year students.

In class, students always tend to find other ones that they better connect with and that will be supportive with them. So I think the [clinical group] had kind of bonded. They had been together before, so that by the time we were having a go around the room to introduce themselves, they already knew each other. They just didn't know the other students in class. And if I think beyond their clinical group sometimes I see the fellows... and I say fellows just kind of as another term rather than male student...they need defined simpatico and common and comfort, in a face that looks like mine. 
Marcie did not provide full descriptions of how the men interacted with faculty and female peers in class: “I don’t remember specific instances or interactions with one another." However, she did indicate that in her experience it was typical that male nursing students interact with each other more closely than with female nursing students. "Yes, most definitely, even so far as having study groups together and not excluding women. Definitely it was like there are students who are seniors right now going into their integrated practicum and of the four [men] that I had, three of them definitely seemed to bond more." In-class observations showed that the men were treated equitably by their faculty and similarly to their female peers. They were provided with the same type of instruction, had the same opportunity to ask and answer questions, and were known by their faculty. I observed that each of the faculty used gender-neutral language and did not exhibit any signs of discomfort with interacting with the men in class.

Summary of faculty and male nursing student interactions. Themes from the evidence related to how faculty interact with male students indicated that the faculty focused on the personality types of the men and that Cliff and Jack’s personalities appeared to be a determining factor in how they engaged with other female students and responded to class content. The faculty all seemed to notice that Cliff was more outgoing and extroverted and that Jack was not outgoing and introverted, consequently presenting an instructional challenge for faculty. They also noted that the men sat together often and that they had connected with each other. One instructor suggested that the bond between Cliff and Jack was natural and was based on the familiarity of being male in a female-dominated environment. Overall, faculty did not seem to have any concerns or 
issues with this pairing and had seen this type of grouping by gender with previous students.

\section{Administrator Perceptions of their Interactions with Students}

As director of the undergraduate program, Vicki supervises the instructors that teach Nursing 210 A\&B. Vicki did not teach in Nursing 210 A\&B and had limited or no contact with the students directly. However, she was able to observe the students in Nursing 210 A when she and other administrators were invited to observe the students present a skit. This class vignette included all members of the class and provided Vicki with the opportunity to see the students interact with each other and their faculty member, Susan, in a positive manner. In addition to seeing the class during that presentation, Vicki reported that the faculty of Nursing 210 A\&B had given her the impression that this was “a good class with a lot of great students.” Because she did not have direct contact with the students in the study group, Vicki was not able to comment on the interactions that the male students had with each other, their peers, or their faculty members. However, the fact that these students engaged in a class skit in front of viewers from outside the classroom showed that the men were integrated and included in this class activity.

\section{Summary of Theoretical Proposition 1}

The evidence shows that Cliff and Jack were concerned with finding another male student to bond with and that making connections with faculty was a central focus for both of them during the first term. The men appeared to immediately have a connection with each other, while making connections with their female peers took a little longer. 
Cliff experienced being excluded from an interaction where a group of women shared stories about their personal lives, and both men expressed discomfort with partnering with women while practicing clinical skills in lab. They also expressed feeling pressured to volunteer in the clinical setting and believed this was due to social mores that men can more easily expose their bare chests in public. The men readily expressed that they liked and felt supported by their faculty and did not express issues or concerns with their interactions with their faculty in either setting. Faculty described Cliff and Jack based on their personalities and interactions within the classroom and noted that they often sat together and had an instant bond. The lone administrator in this study had limited contact with the students but saw a class activity where both men were active participants with all the others. The lack of direct contact with the administrator can be seen as a positive indicator given that the administrator is typically involved in issues of complaints or disciplinary action.

\section{Theoretical Proposition 2: Men Experience Barriers in the Educational}

\section{Environment}

The literature hints at problematic representation and reflection of maleness in the nursing field; it also suggests that males can be excluded in the language surrounding the terms, examples, and descriptions used in nursing (Alfred, Yarbrough, Martin, \& Garcia, 2011; Dyck et al., 2009; Roth \& Coleman, 2008; Smith, 2006; Stickney, 2008).

Interviews, observations, documents, and researcher memos were analyzed to identify how gender was described, represented, or reflected within the classroom setting. An assumption of this study is that feminine gender norms are used exclusively to the 
detriment of male students. For example, nurses being referred to only as "she" in the classroom or in class examples that assume that all students have the same background knowledge of pregnancy and birth. The following section contains a fluid discussion about the evidence gathered around this theoretical proposition, which was organized by the sub-parent codes of 1) faculty use of inclusive language, 2) mother-baby content, 3) men sharing less, 4) men assumed to be physicians, and 5) excluded from mother-baby units.

\section{Faculty Use of Inclusive Language in the Classroom}

Throughout this researcher's observations of class, the learning materials and terms used in lectures were both gender-neutral and inclusive of men and women. This observation was corroborated with all three faculty members, who used he and she pronouns when referring to nurses or nursing during class and in our interviews. This awareness of using these pronouns when referring to nurses was also highlighted by Vicki, who indicated that "those inclusive pronouns need to be used whether or not men are present. That needs to be something that we use all the time.” This showed an administrative awareness of the need for faculty to use inclusive language in the classroom. Vicki also indicated that gender-neutral expressions did not come automatically for her and was something that she still had to work on. "The other day, I caught myself using the female pronoun when I was referring to nurses and then hesitating for a minute and saying, 'and men,' 'or and him,' or whatever. And it was, like, that was not good, and I went back and redid, but it was obvious that my first thought was I was referring to women, right?” Like Vicki, Marcie commented on the 
inclusive nature of the PNMU nursing program and linked these egalitarian dispositions to the leftist tendencies of this particular community. "I feel like politically [our area] is very liberal and we try to be very inclusive of different people and different cultures and all those kinds of things. Other places I think would do it differently.” These comments are congruent with what was observed in the class as well as with the analysis of the academic documents where the use of pronouns, in-class examples, and textbooks reflected gender-inclusive language in the academic environment.

\section{Men and Mother-Baby Content}

While the language and examples used by faculty in the classroom were genderinclusive, the course content of Nursing 210 A\&B did focus more on women and highly gendered discussions about pregnancy than it did on men and boys. This "mother-baby content” or focus on mothers and children is expressly noted as the course content in the catalog described as "populations studied in the course include children, adults, older adults and the family experiencing a normal pregnancy.” While mother-baby content is foundational to this first course that all nursing students in this program take, Cliff and Jack said that they had a harder time with it than other topics and that they had less experience or awareness of issues related to the abuse of women than their female peers did. As a person without children, Cliff discussed his difficulty with learning the differences between the developmental ages of children.

There were examples like learning about children's developmental stages that was hard for me because I'm not around a lot of kids. I can't even tell what age kids are in general already. When you don't have any visual cues or experiences [about children's developmental stages] it's hard to remember for me and to really stick to it and then some of the women's health promotion things. 
Cliff explained his frustration further:

You know, the maternity stuff is hard because both of us are men who have no experience in that and we don't have friends or family members who have gone through that experience where we say okay, yeah, I've been there, seen this, and this is what it's like. So that's definitely a disadvantage. And the other disadvantage is that neither of us are fathers.

Finally, Cliff also suggested that content related to physical abuse was focused specifically on women. "You know the scenarios where we were supposed to be asking women in every clinical scenario, are you abused at home? It made me wonder should we be asking men that. I know the statistics don’t prove that you should be asking guys this question but maybe we should be. There were things like that that would cross my mind from time to time. Cliff also made a point to mention that he thought that "Susan was really careful to mention that it happens to men too, but the focus was on women and children.” It could be argued that there are limited ways to cover mother-baby content and physical violence against women without taking a female-centric approach, but the result is a predictable level of discomfort for male students.

Jack also talked about his discomfort with some of the mother-baby content. “And children, I don’t have that much experience with children. So that was difficult. I think I couldn't relate to the kid stuff. That was all new for me. So it's...hit and miss.” Despite noting this difficulty with the mother-baby content, Jack observed that he had the same opportunity to learn the information as any other student. "It's our responsibility. I was provided with the same information. I was provided with enough information to be successful at all the simulations. I just needed to do more work than others might have had to do." 


\section{Men and Less Personal Examples about Parenting}

As found earlier, the students and faculty have different perceptions about what is happening in the classroom. While male students described their lack of experience and knowledge with mother-baby content as a limitation (in Cliff's case) or a challenge (in Jack's case), faculty noted that both men tended to share less about their own experiences with parenting in class than their female peers. Susan said, “I don’t ever hear men bringing up that experience around children in the way that women do" Marcie agreed.

Yes, my students with kids would talk about their kids but the male students don't volunteer as many stories about struggles they had and that kind of thing. I knew that they had kids and they kind of talk about them but it wouldn't be sharing clinical stories necessarily. One time, this was years ago, a student had a child with ADHD and so then we were talking about that. He talked about his specific experience with that.

In fact, faculty noted that men did not tend to share much about parenting in class, yet they did not seem to show any lack of comfort with the mother-baby content. This contradicts what the men stated about their discomfort in their separate interviews. The observation notes, academic documents, and researcher memos for the case study do not shed further light on the struggle that men encountered with the mother-baby content.

\section{Assumed to Be a Doctor}

A particular area where gender came up in a differential manner was in the clinical setting while doing head-to-toe exams on children in an elementary school clinical setting. Jack noticed that when he was working in this setting, the children assumed that he was a doctor. 
I felt a little singled out during the screenings for children because I was the only male out of all the females [nursing students] at the school and I obviously look different because I'm wearing slacks and a button up shirt. I looked more 'doctorly' according to what everybody else said and I didn't even come into [the clinic] thinking that it was gonna be an issue. Then all the kids were talking not necessarily to me, but to a person whom they believed to be a physician. For example they told the faculty, like Lisa, 'I want the doctor to look at my teeth next. I want the doctor to sit next to me during lunch.' I felt singled out in this scenario.

Jack explained that he told the children that he was a nurse like all the other people there and that men can be nurses too. Jack's discomfort increased when his female classmates heard the children misidentify the men as doctors. "All the other nursing students that I was with were talking to me about how all these kids thought that I was the doctor and they were the nurse. So in that whole scheme of things I guess kids may still think that culturally.” Similarly but in a slightly different way, Cliff had an experience where he and his fellow nursing students were assumed to be doctors by the children they were working with due to his attire.

They all thought we were doctors because we had the white coats on. So they were like 'go doctors,' and we replied 'no actually we're nurses' but it went really well [because] they seemed excited and they were definitely engaged. I didn't experience any discomfort on the part of the kids and they all seemed like this was normal, this is healthy.

In both instances, the men attributed their experiences as much to their attire in the clinical setting as they might have to their gender. Jack also showed awareness that the assumption that he is a doctor might occur again.

I haven't been exposed to another demographic that might not be that educated to realize that men are nurses. I know kids don't have that in their head, but I might come across that way in clinical so I'll deal with it as it comes and I will let them know. I'm trying to think. I had a nurse that was a CNA instructor when I did my CNA course and I think that he had the same thing. He'd walk into a room and they thought that he was the doctor. So we'll wait and see if that happens. 
The instances of being misidentified as a doctor occurred in clinical situations outside of the direct classroom and were not observed by this researcher. None of the reviewed academic documents showed any acknowledgement that male nursing students might encounter this phenomenon nor was there instruction or direction from faculty, administrators, or within the curriculum about how to react should this event take place. However, while both Cliff and Jack were perceived by young school children as doctors, female classmates dressed in hospital white coats were similarly identified.

\section{Anticipated Exclusion in Some Clinical Experiences}

There were other areas of significant gender difference in clinical experiences.

Cliff shared an example of a time he was asked not to provide care to female patients in his role as a clinical nurse assistant.

I did experience at this local hospital...there were quite a few female patients that did not want me around. It kinda hurt my feelings a little bit but at the same point I understood. I know there will be a chance that I may not be allowed to work with females in certain scenarios. They may not want me there.

Cliff's realization of the likelihood of a repeat experience indicated a level of acceptance that he would experience certain gender barriers as a nurse; this understanding was echoed even more strongly by Jack. In fact Jack spoke in clear and pragmatic terms:

Yeah, there are gonna be a lot of settings that gender is gonna play a role like when I'm doing treatment for patients. If a young woman doesn't feel comfortable with having a male nurse or whatever, what do you do in that situation? Hand it off and get a different patient, you know?

Although Cliff and Jack were provided with the same opportunities in the clinical setting as all the other students, both the students and faculty in the study acknowledged that 
men may be excluded from certain clinical experience especially related to mother-baby content. Faculty member Polly mentioned that men are not welcome in mother/baby units. "I have friends that are nurses who are male saying they were getting a lot of push back from people who disallow treatment because they are men.” Faculty member Susan stated, “The truth is they're not as welcome in maternal child work, that kind of thing. I know that when I talk about pregnancy and so on, we just get going in a way that can exclude men, and people who are not familiar around that process.” Administrator Vicki, shared this same sentiment. "I think that absolutely in the practice world that there are some practice areas where men are less welcomed. I've heard people talk about men in midwifery and how that is not necessarily something that's desirable." While both the men and faculty acknowledged that men may be excluded from certain clinical settings, the researcher's review of classes and course documents revealed no specific conversations or curricular content that would prepare male nursing students for how to handle this experience.

Without any kind of preparation, it comes as no surprise that Cliff and Jack had not talked with their faculty about sensitivities concerning practice rotations on female patients and how to handle rejection based on gender. When asked about what discussions, if any, the faculty had had about preparing male students for clinical rotation rejection, Vicki said she couldn’t speak for other faculty members but noted:

When I taught in Mother Baby and had men on the unit, I would specifically take them aside and talk to them about their feelings because they weren't always selected to be in postpartum. When they were thrown in there, I talked to them about their comfort level and about the possibility that a patient might refuse them as a nurse because they were a man. 
Vicki was unaware of any strategic effort on the part of the faculty or the administration to actively address this issue in the clinical experience in a systematic manner. Vicki’s individual effort notwithstanding, it appears that the PNMU nursing program is missing an important opportunity to teach male students about diplomatically negotiating with female patients who are distrusting of male nurses.

While the faculty used gender-inclusive language in teaching situations, they did not seem to be aware of the heightened discomfort men feel in the clinical setting when working with female classmates. None of the faculty identified additional barriers that the men in Nursing 210A\&B faced beyond the specific professional areas where they were not welcome to practice.

\section{Summary of Theoretical Proposition 2}

Cliff and Jack enjoyed classroom settings where gender-neutral language was consistently used and encouraged. However, they experienced some barriers. They were misidentified as physicians by the children in their clinical rotations instead of as nurses and they were excluded from providing care based on their gender. They also expressed less familiarity and less comfort with mother-baby course content and the faculty also noted that men tended to share less about their own experiences with parenting.

Faculty seemed to focus on how the men interacted in the classroom and construed their lack of sharing about parenting as markedly different from what was shared by their female classmates. It is also significant that faculty did not appear to notice the men struggling with mother-baby content. Students, faculty, and the one 
administrator acknowledged that there were certain clinical experiences in which men may be excluded. There did not appear to be any preparation for helping students address this issue when it occurred, and the tone from the faculty was one of acceptance that this type of exclusion should be expected. In addition, there was no mention of the dilemmas of ethical nursing practice that these exclusions present.

\section{Theoretical Proposition 3: As Gender Minorities, Men May Not Be as Academically Successful in a Female-Dominated Educational Environment}

A core assumption in the literature is that barriers experienced in the educational environment are detrimental to the academic success of men in nursing (Anthony, 2004; Kenney, 2002). In this study the data from the observations, academic documents, and researcher memos were analyzed to identify what if any differences in academic achievement were occurring for men. The following section describes how being in the spotlight and feeling pressured to volunteer in the educational setting alternately spurred the men to do better as well as triggered their withdrawal from in-class interactions.

\section{In the Spotlight}

Cliff and Jack described a number of situations where they perceived administrators, faculty, and female classmates to be acutely aware of their maleness. Cliff thought that he was let into the program because there were not enough males. "I have a joke that I say about getting into the program because I was on the wait-list here and I got accepted, and once I got in here, it was just Jack and me. I was like, well maybe they just felt bad because there was only one guy so let's let this other guy in here.” Cliff believed that there was an increased value placed on his maleness by PNMU 
administration since more males were needed in order for the program to achieve its diversity goals. Cliff, therefore, jokingly construed his value to the nursing program as adding to male student numbers rather than the potential quality of his work. In addition, the physical attributes of the men were identified and valued according to Jack, who shared a story where Cliff's height had been called out. "I noticed Cliff's gender was pulled out, because he is tall.” Jack explained that in theory class, the instructor, Susan, shared that she was initially guarded around Cliff because he was tall and she perceived his height to be imposing to her. Cliff also commented on this experience, indicating that others in his class may have refrained from approaching him because of his height. "I am a tall man and I don’t think that I come across as being scary or intimidating you know. But I know some people probably feel that way just because of their past experiences or maybe something about me triggered something for them.” He also shared that gaining an awareness of how others viewed his height had been helpful to his practice as a nurse when working with patients.

I was making sure to get down on one knee to be able to be at their eye level or just kind of hunch down a little bit and Susan saw me doing that and she was like 'I'm so proud of you', you know? I could definitely tell that people are more open with me and seemed more friendly when I do that to show that I'm acknowledging that I need to make an attempt to kind of be on the same level, make sure the playing field's leveled.”

Cliff's practice of self-conscious physical leveling helped him confront whatever latent fears his fellow students and patients might have regarding tall men.

Another way that the men were more obvious to those around them was when they were absent from class. Jack observed: 
I had to miss a class last term, right, because of personal reasons. And I noticed that other people miss classes here and there too. And it went unnoticed. But for me I feel like I'm under a spotlight kind of because just naturally I'm different than everybody else. And when I missed my class, I received a ton of emails from and text messages saying things like, 'Where are you at'?

Jack said that when he returned to class, "I felt singled out because the teacher said something to the effect of 'Oh, nice to see you that you're back.'” Although Jack said that his absence was more visible than when women classmates skipped class, this increased visibility was a benefit to him. "I think that being under a spotlight, whether that's the case or not, because I can’t read other people’s minds, is an advantage for me. It gives me an advantage because it makes me try harder and it makes me more accountable.” So in this way, the extra attention helped Jack participate more in class.

While both men identified situations where they felt that they were noticed more than others in their class, they both indicated that this awareness was actually helpful for them.

\section{Feeling Pressured to Volunteer}

Cliff and Jack shared examples of feeling highly visible in clinical coursework. Cliff said that he and Jack noticed and discussed feeling pressured to volunteer as practice patients during clinical because it is more acceptable for men to show their torso in public. “In clinical, Jack and I both talked about this and we felt we're a little more obligated to volunteer.” This statement corresponds with what was observed during a Nursing 210 B class when faculty asked for volunteers "for the head-to-toe exam who doesn't mind having their chest exposed.” Then the faculty member clarified, "not fully 
exposed” in a way that was clear to indicate that the full chest didn’t have to be bared.

This researcher observed a female student next to Jack "nudging him and suggesting that he go up.” Surprisingly, both men declined and after a few minutes of waiting and no students volunteering, another faculty member in the class volunteered to be the patient. While this example does not indicate that the faculty members were pre-identifying the men to participate, this example may indicate that some social mores related to exposing skin in public are present in the clinical setting. It also suggests that it may be easier for men to offer themselves as practice samples in ways that are not comfortable for female students. A reasonable assumption could be made that this pressure to "participate" weighs on both Cliff and Jack in addition to any pressure they feel related to coursework or their ability to succeed in the program.

In fact, Jack specifically addressed this pressure to volunteer as a practice patient in the clinical portion of the coursework. "So there was one time that nobody wanted to volunteer and somebody was like oh, you know Jack, Cliff, one of you guys just do it. You know. And it's like ...don't single me out, you know.” Thus, the other students in the class were volunteering Jack and Cliff for an activity that nobody wanted to do and Jack felt singled out by this interaction by his female peers. It also seemed that Jack bore resentment about these suggestions.

The researcher also saw Jack and Cliff actively looking down at the materials on their desk during the call for volunteers and not engaging with those around them. In short Cliff and Jack seemed to be resisting or rebelling against the call to volunteer. They appeared to be avoiding making eye contact with the faculty or with the other women 
around them until the faculty had moved on to another topic. The observation of this inclass exchange was the source for new follow up questions with the faculty regarding the expectations for students to participate as volunteers during in-class demonstrations.

\section{Academic Success Not Based on Gender}

The men appeared to be aware that they were joining a female-dominated profession but did not seem concerned about how that dominance would impact them. As one of two men in the class of 36, Jack shared that his educational experience so far had been better than average. Cliff indicated that he too had had good experiences to that point.

I don't feel excluded or anything [and] I feel like I know that Susan was more sensitive, she would definitely try and include Jack and I in things plus ask us questions about our perspective from time to time. I think that gender plays a very minimal role but ultimately it's how the individual is and what their strengths are that determine if they're gonna succeed in nursing or not. I think that it doesn't matter what your gender is. We can all do the same job; I think women are definitely given that role of being the more sensitive and more nurturing but that doesn't mean that men can't be that way either.

Jack also indicated that he didn’t have any concerns going to school in a program dominated by women. “I don’t notice it at all unless somebody brings it up and then I kind of look around and I'm like okay I am around a bunch of females, you know, but I guess I see them more as people.” These statements make it difficult to ascertain if both men were providing complimentary responses to the program in order to protect their student status. Additionally, both tended to minimize the importance of gender within the program, focusing instead on mastery of course content. 
While the men minimized the impact of gender, faculty readily expressed awareness that nursing is a female-dominated field and that men are underrepresented in nursing. Susan said, “The field is very estrogen soaked” and Polly added that "men have to come to terms with being a male and coming into a woman-dominated profession.” However, along with this awareness that nursing is still a female-dominated profession, the faculty also expressed a desire to have men in the nursing program at PNMU with Vicki saying: "I really think that men in nursing add a huge richness to our profession and I think that they are an important subgroup of our profession.” Polly also stated that having more men in the program will "shake us up too."

The faculty discussed holding men to the same expectations as the women with Marcie stating, “I’ve seen people make assumptions about male nurses and I'm like whoa. You need to think of him [the student] the same as every other nursing student.” Vicki expressed the same sentiments.

I think we really need to watch what our expectations are and that we really need to make sure that we're talking about professional expectations for all students. I try to think of students as students and not as female students and male students. I think that to a degree, I try to do that so much that I maybe don't see the differences. Absolutely, I would say that they're equally successful in nursing school.

Cliff summarized his experience at PNMU thus far by saying, "I think that just because the numbers of men aren’t high, it doesn't mean that PNMU isn't accepting or anything like that. I definitely feel accepted and I feel included.” Jack also indicated that he thought his faculty had treated him equitably in the Nursing 210 A\&B course. "I think 
they treated us all neutral. We all have the ability to care for people. So I think they did a good job. I don’t think they tailored it more towards women or anything like that.”

\section{Summary of Theoretical Proposition 3}

While the men may have perceived that others were more aware of them in the classroom setting, they indicated that this additional visibility in the classroom helped their academic progress. In addition, neither the students nor the faculty indicated that gender was a barrier to success in the nursing program. However, the men also appeared to minimize gender as a factor in their educational experience but they did encounter some barriers like feeling pressured to volunteer, being uncomfortable partnering with female peers, and being excluded from clinical situations, all of which appear to be gender-related. The faculty also seemed to treat the men the same as the women in the class and gave them the same opportunities to learn; however, they appeared to have limited knowledge of additional barriers, beyond using inclusive language, for men in the educational setting. At the end of the term, both Susan and Polly indicated that the men passed each section of the Nursing 210 course and it was subsequently confirmed by both men during their second interviews that they had each passed their courses in the Fall Term. 


\section{Conclusion}

An initial review of the data through the theoretical assumptions of (1) men are isolated from faculty and peers in nursing school; (2) men experience barriers in the educational environment; and (3) as gender minorities, men may not be as academically successful in a female-dominated educational environment showed that these initial assumptions were not always verified by the evidence. Contrary to the assumption that men would be isolated from their faculty, the evidence showed that the men felt connected with their faculty, made these connections early, and identified their connection with their faculty as an important part of feeling supported in the educational setting. The evidence did find that men experienced social isolation and exclusion in the classroom from female peers and had less familiarity with the mother-baby content. However, the men did not associate the faculty with these barriers; and in some cases, they noted that differential treatment in the form of being more visible and more pressured to volunteer in the classroom was beneficial to their learning experience. Finally, the assumption that men may not be as academically successful as their female peers was not in evidence as both men and all the women who completed the course did pass both sections of the Nursing 210 A\&B course.

A key portion of this study was to review what is happening in the educational setting for men. These findings show that some of the initial assumptions were not supported by the evidence. While the data suggest that the men felt connected to their faculty and were academically successful, some barriers did emerge that need additional consideration. In addition, the question of faculty awareness of gender issues in the 
classroom and how these barriers may impact the educational setting need additional review. These questions are addressed in Chapter V. 


\section{CHAPTER V: DATA ANALYSIS}

According to Yin (2009), part of the analytic process includes “comparing alternative descriptions or explanations of the same case” (p. 176). This chapter reviews the evidence through the lens of the research questions to compare the results with those identified in Chapter IV. "The purpose of the repetition is to show the degree to which the facts fit each model, and the repetitions actually illustrate a pattern-matching technique at work” (Yin, 2009, pp. 176-177). Comparing the data to both the theoretical propositions as well as the research questions allows the data to be examined from multiple perspectives and creates opportunities for alternate perspectives to emerge.

Yin (2009) suggested that there are "specific analytic techniques to be used as part of and along with any of the general strategies” (p. 136) when evaluating data. These five techniques include "pattern matching, explanation building, time-series analysis, logic models, and cross-case syntheses” (p. 162). These five analytic techniques are meant to help focus the researcher in developing a "systematic sense of what is worth analyzing and how it should be analyzed” (p. 162). Yin (2009) described the analytic technique of pattern matching as the process of "comparing an empirically based pattern with a predicted one” (p. 136). The explanation building analytic technique is designed to help "stipulate a presumed set of causal links about it or how or why something happened” (Yin, 2009, p. 140). The time series analysis is used in “experiments and quasi-experiments” (Yin, 2009, p. 144). The logic model "stipulates a complex chain of events over an extended period of time (Yin, 2009, p. 149), and the cross-case synthesis 
technique "applies specifically to the analysis of multiple cases” (p. 156). Regardless of the analytic technique chosen, Yin (2009) stated that "your analysis should show how it sought to use as much evidence as was available, and your interpretations should account for all of this evidence and leave no loose ends.” (p. 160).

Through this second round of data analysis, pattern matching was used to compare the evidence with the research questions that evolved during the course of the study. Although similar to the original research questions, these questions emerged as the interviews and in-class observations occurred and shifted to (a) understanding the nature of the interactions between nursing faculty and male nursing students in the educational setting; (b) examining how gender is described, represented, or reflected within the classroom setting; (c) reflecting the perceptions of visibility, polarization, and assimilation identified in Kanter's (1977) theoretical framework of tokenism; and (d) identifying to what extent faculty are aware of gender barriers in the classroom.

In order to evaluate the key findings from the first round of analysis and gain a deeper understanding of what occurred within the setting, each of the key findings that emerged from viewing the data through the lens of the individual theoretical propositions was re-reviewed through the lens of the research questions. This researcher created a matrix to sort, order, and categorize the evidence from the key findings in order of how they related to each of the research questions. In this way the evidences from the interviews, observations, documents, and memos were re-sorted to are re-presented within a different structure to compare and contrast the findings with the research questions and to search for alternate explanations (Yin, 2009). 


\section{Nature of Interactions of Faculty and Students}

It can be argued that faculty are responsible for creating the learning environment in which ideas, perceptions, skills, and thinking about the course content are established for students. The literature also currently suggests that faculty can unknowingly perpetuate the feminization of the nursing role within the educational setting, sending a message to male nursing students that they are not welcome (Crow et al., 2005; O'Lynn, 2004; Roth \& Coleman, 2008; Smith, 2006). As gender minorities within a course where all faculty members were women, it is important to clarify the nature of the interactions between the male students and the female faculty to better assess how these interactions may be contributing to a welcoming or unwelcoming educational experience for the men. For this research question, the evidence was arranged into four patterns: 1) Faculty are a helpful, supportive resource; 2) Faculty see no differences; 3) Benefits of extroversion versus deficits of introversion; 4) Emotional withholding and experiential gaps. From these four patterns a larger picture of how faculty and student interactions shape the educational experience for men in nursing emerges.

\section{Pattern 1: Faculty Are A Helpful, Supportive Resource}

Class observations showed that the faculty for both sections of Nursing 210 A\&B interacted regularly and similarly with all students in the class; male and female students were not treated differently. The researcher observed that Susan was surrounded before class by students who were talking with her or asking her questions. The men more often were seated at their desks during this time; however, Susan would interact with the seated 
students as well. Both Cliff and Jack and other women in the class received greetings or small acknowledgements in a similar manner. Susan knew the names of most students in the class and called on both men and women in the class regularly. At the end of each class, Susan would again be engaged with students and other guest faculty members, staying in the classroom for many minutes after the class was over. The men sometimes engaged with Susan after class, but they didn’t tend to stay after the class very long; and Cliff and Jack tended to leave together when class was over.

These observations suggest that the men did not seek out additional socialization or interaction with Susan pre- or post-class time, like some of the women in the class; however, their in-class interactions with her were neutral or positive. This could indicate that men did not seek out their faculty in the same way that women did or it could suggest that there are gender differences in the way that men and women socialize with their faculty in class. However, not all female students would approach Susan before or after class so this could have been unique to this class and could be an interesting question for further research. For her part, Susan also appeared to make sure to engage with the students even if they did not approach her before or after class, indicating she was comfortable allowing students to approach her as well as with approaching the students.

Cliff and Jack portrayed their interactions with Susan as helpful and engaged. Cliff stated, “I really liked Susan’s teaching style.”

I knew in the first day of health promotion, just her presence and just how she is, this teacher is gonna be awesome and I think that this is gonna be a great class and she just has a great presence and she's funny and she's just really, you can tell she's really honest and she's, everything she says you know it's true and she's speaking from the heart and she means well in every opportunity and I really 
respect her a lot and I know for some people, she was kind of, that teaching style is really different and some people have a hard time but everyone liked her.

Jack described his interactions with Susan this way: “I haven’t talked to her personally in any office visits or anything. So it's been pretty superficial, but what she says is impactful in every class. She’s a good teacher.” In addition, both Cliff and Jack gave examples of interactions they had with other faculty members. Cliff stated, "I really like my faculty. I feel lucky because I feel that we have some of the most friendly and um, helpful faculty.” Cliff also stated, "I'm very happy about all the instructors, I feel like I have a good connection with all of them and my cohort, except for the last little few people that I haven't been able to get to yet, it's great. I'm very happy.” Jack also described his connection to one of his clinical instructors. "Jillian is my clinical instructor for my clinical group and she’s prior military [like Jack]. She was a nurse in the Army so we have this like instant bond I think, so she tells me that she knows all these people who she can introduce me to and they have all these resources.” Jack also stated that "the teachers are compassionate and they seem to really care about us which is really cool.” Thus, Cliff and Jack not only believed that their interactions with Susan were positive but that their interactions with the other faculty members in both Nursing 210 A\&B sections were positive as well. The men believed they were not treated differently in this course and that faculty at PNMU were helping to create a positive learning environment for them. Overall the men described their interactions with their faculty as helpful and supportive, and as a resource; and their comments indicate that they had positive interactions with their faculty throughout the term. Their comments aligned with the researcher's observations of the classroom environment. Finally, both 
Jack and Cliff perceived their interactions as inclusive and their connection to faculty as affirming.

\section{Pattern 2: Faculty's Cautious Comments of Small Differences}

The interactions between the faculty and the male students appeared positive; the researcher observed Susan, Polly, and Marcie engaged with all students as part of giving and assessing instruction in a constructive manner throughout the term regardless of gender. Faculty comments about students were positive and related to the course content. When asked about their awareness of any differences in learning or practicing nursing based on gender, the faculty spoke more globally and indicated that they did not find differences based on gender but based on their engagement with the student. This was exemplified in Susan’s response to a question about student learning styles. “I don't. I mean I don’t find them [men] either ahead or behind. Of course [they don’t learn] at the same time, ever. And it's hard even - with this many students, who knows what's going on inside their heads. You know, I get what I get, in terms of assignments, and then I meet with a lot of them individually and I can hear it.” Susan accepts that learning occurs at various rates for students and that she does not always know how students are progressing until she talks with them directly.

Marcie also talked about the students broadly in terms of assessing their learning and she did not see differences in learning styles or how perceptions about nursing changed based upon the students' gender. When asked about any differences that students may have entering nursing based on their gender, she said, "Their image of nursing based on their gender? Not really. I mean it feels like they as a group come in 
very focused on the in-patient kind of practice focus. I see that with both genders I guess.” Marcie saw new nursing students from both genders coming in with a narrow view of the scope of nursing practice and didn't see differences in how new students viewed nursing based on their gender. She also found it difficult to comment on any differences between the female students and Cliff and Jack and stated, "Well, there are only two. They weren't in my clinical group unfortunately. I didn’t get to see that.” Marcie did not note gender differences in how the male and female nursing students performed in class. It could be that she didn't notice any differences or that she felt uncomfortable sharing her observations with the researcher. Additionally, Marcie might have been purposefully minimizing her observations of gender differences between the men and women in the class as a way of ensuring that she does not treat students differently and role modeling her values for treating all students equitably. In class observations showed that Marcie addressed the entire class and responded to students that spoke up or asked for additional clarification in a positive and forthcoming manner. Cliff and Jack tended to remain quiet during Marcie’s lectures.

This was true for Polly as well; she stated that she had not really seen any differences in the way that males approach nursing. Instead of referencing differences based on gender, Polly described the differences between students based on their ability to take ownership of the nursing role.

I tell them right up front, in clinical, that you are developing your practice as of day one. Everything you do is toward your practice. The sooner they own what it is they need to do, they feel responsible and accountable. So you can see [student learning] as an accumulation and evolution and usually students get the aha somewhere in the process. They realize that it is their practice, which they will 
have a license for and some get there sooner than others. But you see when it takes off, it's like...it is really there, as opposed to this thing that they're just sort of putting on, and trying to perform.

Polly assessed student learning based on their comfort level with their nursing skills in the practice setting and their ability to perform without seeming to fake their abilities or act as if they were a nurse. Polly also noted that the students are provided with the same information about career opportunities within nursing without assumptions about gender. “Assumptions - no, I think they [the men] also have the opportunity to look at and try on and think about other possibilities. At least that's how we really try to make this course available to all students, and so I wouldn’t think that it would be any different.” Polly expressed her value that students should be treated the same regardless of their gender and indicated that the faculty at the PNMU are consciously thinking about how to present course content in ways that are equitable for both men and women in the class. Thus, Polly was aware that men may experience some barriers within nursing and was conscious of trying to present career options within nursing abased on skill and interest and not based on gender. However, Polly's inability to identify differences between the men and women in the class could also indicate her reluctance with sharing her opinion with the researcher.

The faculty noted few if any differences in the way that Cliff and Jack learned but they cautiously noted differences in the clinical setting where male nurses are not as welcome and at least one faculty talked about how students need to embrace certain aspects of the nursing role in order to be successful. The three faculty participating in this study may not have noticed differences between Cliff and Jack and the women in the 
PERCEPTIONS OF MALE NURSING STUDENTS IN NURSING EDUCATION 102 class or they may have minimized any differences that they did see when talking with the researcher for their own reasons. Finally, the small number of men in the class may have limited the opportunities for differences to be more visible to the faculty. Ultimately, gender differences were identified in the frequency and limited ways that Cliff and Jack shared in class and with an awareness that men will be unwelcome in some clinical areas. However, these differences were not visible to a point that hindered Cliff and Jack's academic success in the class.

\section{Pattern 3: Benefits of Extroversion versus Deficits of Introversion}

The faculty spoke broadly about the ways in which students learn and did not speak directly about the ways that Cliff and Jack learned in comparison to their female peers or specify differences based on gender. However, the faculty did reveal that their in-class interactions with Cliff and Jack helped them assess how these men were learning. For example, Susan described Cliff as “pretty extroverted, pretty intense. You know? Wants to get it right” while she Jack described as “also like very awake and alert and very present in class. He's not nearly as talkative as Cliff.” Polly revealed similar thoughts. "Cliff was more forthcoming, so I could know where he was at with something” and "my sense with Jack was just that he maybe just got more comfortable over time” speaking up in class and interacting with the faculty. Marcie also reflected on her interaction with the men. "Cliff was very vocal. He felt really comfortable speaking up” and “Jack, I don’t really know, because he didn’t even talk much when I would go and talk to their group.” The finding that the faculty are assessing learning based on inclass interactions underscores the importance of communication in the classroom and 
highlights how differing communication styles could impact student learning. Cliff and Jack communicate differently in class with Cliff providing more opportunities for direct feedback as a result of his openness with faculty while Jack required faculty to engage him directly instead of initiating contact with faculty on his own.

Marcie could tell that Cliff was internalizing the role of nurse and learning how he had a role with challenging stereotypes by how he used self-reflection to modify his behavior in the clinical setting.

I feel like Cliff was very reflective and he would share. When [he was in clinical with school] kids he came up to me and he was like 'oh my gosh I couldn't believe it. I went up to this kid and I was so how was your experience with being in the school screen. And he [the elementary student] was 6 and so he just kind of looked at me like huh? And I realized oh my gosh I have to change my language.' So it was that kind of thing where I could see that he was reflecting in action.

Marcie had a harder time knowing how Jack was progressing with the course content because he was much quieter and would not speak up when she approached his group. Jack’s limited in-class engagement with Marcie made it difficult for her to assess his learning. Jack’s failure to speak up when Marcie approached his table was a missed opportunity for him to show how he was thinking about the course content and did not provide Marcie with an opening to provide him with needed feedback. This is an important aspect of the in-class dynamic that is occurring within the educational setting and could be a limitation in Jack's overall academic success. Polly also commented on the differences in the way that Cliff and Jack interacted with her in class.

One is very vocal and the other not so much. Jack was hard to unpack. I couldn't always tell how well he was doing because he didn't give a lot of eye contact in 
class and I thought to myself, is he dismissing this content? I would also think to myself that he might already know this because his exposure in other aspects of healthcare? And so then I would try to ask a question later to sort of see how he was doing. By contrast, Cliff is like an open book being more forthcoming, which made it easier for me to know where he was at with something.

Polly seemed to be concerned about Jack early on because he was less expressive in class and did not seem as engaged with others. However, when she investigated further by asking Jack about his learning, she was able to assess that he was where he should be with understanding the content. It seems that Polly had a better understanding of where Cliff was in his learning due to his higher level of engagement in the class. As an experienced instructor, Polly made sure to question Jack about the content in order to give him the opportunity to show her how his learning was progressing. A less experienced faculty member might not have provided that opportunity to Jack, which may have had more adverse effects to his academic success. In addition, Jack did not appear to be aware that his lack of engagement with his faculty in class might be causing them to question what he had learned. Jack might not know that his faculty expected that he actively communicate with them in class or that his lack of communication was a deficit that he needed to overcome. It could be that this type of in-class communication is unique to the nursing culture and is not something Jack had encountered before. In addition, this lack of communication with his faculty might indicate that Jack was not as comfortable with his faculty as he had originally indicated.

With in-class interactions used by faculty as a way of gauging student learning, in-class interactions between faculty and students are a critical component to student success. With more outgoing students like Cliff, it appeared easier for the faculty to 
assess how learning was occurring and to provide direct and immediate feedback during class. This ease of two-way communication between Cliff and the faculty provided Cliff with a connection with his faculty and showed that he was actively engaged. Jack’s quieter and more introverted nature made it harder for the faculty to identify how his learning was progressing and it became difficult for them to provide direct feedback to him during class. The faculty had to wait to see what he produced with homework or through tests in order to assess his learning. This lack of in-class communication with his faculty may have limited Jack’s opportunities to receive instruction necessary for him to build his nursing skills. However, the faculty appeared willing to bridge this gap in communication with Jack by asking him additional questions and giving him an opportunity to show what he had learned.

\section{Pattern 4: Societal Norms and Experiential Gaps}

Susan, Polly, and Marcie were clear that there are few gender specific differences in how male students learn or show their clinical skills from female students. However, the faculty did note a few differences in their experience in working with male nursing students. Susan described male nursing students as "maybe more linear and I would say less dramatic. Maybe they wouldn't come to me with the drama that some of the women do. But I haven't experienced the same kind of drama out of the men.” Susan did not find Cliff or Jack to be either ahead of or behind their female peers but she noted that they were less likely to share personal details of their lives and to involve her as an authority in navigating inter-personal issues with other students. This fit with earlier 
PERCEPTIONS OF MALE NURSING STUDENTS IN NURSING EDUCATION 106 patterns that indicated that Cliff and Jack were less likely to engage with Susan before or after class and less likely to share personal stories about their experiences in class.

Marcie also noted she had seen others react to male nursing student's differently in clinical situations and gave an example of a patient asking one of her male nursing students, “Like well what are you doing here?' [and the male nursing student responding that] 'I'm in nursing school. I'm going to be a nurse' [and the patient responding] 'But you're a boy, what?'” Marcie has seen patients challenge men in their role as nurses and question their ability to perform the nursing role. However, she does not seem to provide male students with any preparation that they may encounter these stereotypes. It is accepted that societal beliefs still uphold the role of a nurse as a female-dominated role. Marcie also witnessed male nursing students cope with having their role in nursing challenged. “A student who was a military guy, shaved head, I still remember like his reflection and [the student] just going 'wow what an impact I can have on these preconceived notions that like the 3- and 4-year-olds have.'” Marcie suggested that reflection assignments are a useful tool to help men cope with barriers they may experience in the clinical setting. However, she did not appear to have given thought to how she as a faculty member could help smooth these barriers for her male students and/or address these societal stereotypes with patients directly.

Students come to nursing with knowledge from their previous experiences, which can help provide a foundation for learning new content and skills. However, Polly noticed that male nursing students have less exposure to mother-baby concepts than her female students. 
The idea of [childhood] development is an aspect of what one needs to consider when one is assessing a particular age group. Some [students] that I've seen were less in tune to what level [the patient should be at developmentally], but then again, some of the female students were also that way. And I don't want to make any assumptions myself. But some struggle with it more than others. Some are more open to it, I think, and again, it's how they want to structure or conceptualize the role.

In her experience, Polly has found that male nursing students have less experience or knowledge of children's developmental stages, which may create a steeper learning curve for them. However, she also indicated that this is not solely limited to male students and noted that some women experience this limitation as well. In either case she perceived the students' ability to be successful in overcoming this obstacle as their willingness to realize that this is critical information for them to be aware of in their nursing practice. It does seem though that students who have less personal experience with parenting or caring for children have a steeper learning curve than those who come into nursing with that knowledge and it is important for faculty to make sure that they provide the required time for all students to learn this information.

Susan, Polly, and Marcie did not identify differences in learning or nursing ability for Cliff and Jack based on gender. However, important differences were noted with Cliff and Jack’s varying levels of in-class interaction, their lack of sharing personal experiences in class, and their gaps in knowledge of mother-baby content. In addition, it was acknowledged that men still face societal pressures in the clinical realm of their nursing role. While sharing fewer of their personal experiences in class did not appear to hinder Cliff and Jack’s academic success, their gaps in knowledge of mother-baby content posed a challenge that they needed to meet in order to successfully pass their 
classes. However, one area that faculty appear to be aware of is related to gender stereotypes that may occur in the clinical setting with male nurses. While the faculty did not prepare Cliff and Jack for this type of occurrence, Cliff and Jack need to know how to cope with societal stereotypes about their role as nurses and be prepared to educate others about their ability to provide quality nursing care.

\section{Summary of Interactions of Faculty and Students}

Overall, the faculty tended to cautiously identify communication and engagement differences between Cliff and Jack but they did not describe differences in the way the men approached nursing practice as being inferior to their female peers. The faculty were more forthcoming when sharing their thoughts on the barriers that men more broadly encounter in nursing like having limited exposure to mother-baby content and being challenged in their nursing role by patients; they only cautiously indicated that they had seen these types of differences in Cliff and Jack.

This researcher did not observe interactions between the students and administrator. As the program director for the course, the administrator had no in-class role for either section of Nursing 210 A\&B and also indicated that she had not had any direct contact with the students in this cohort. The administrator did indicate that she had not received any complaints from students in this class and that had the students or the faculty in the class had any complaints, she would have been contacted.

In reviewing the data to better understand the nature of the interactions between nursing faculty and male nursing students in the educational setting, the researcher found that the men described liking their faculty and their teaching styles, and the men 
generally had established positive relationships with them. The faculty-student interactions were mostly characterized by the way the men interacted with the faculty in the classroom and the faculty tended to describe the students based on their varying levels of in-class participation.

\section{Gender Neutrality: Descriptions, Representations, and Reflections}

The literature, suggests that males can be excluded in the language surrounding the terms, examples, and descriptions used in nursing (Anthony, 2006; Denhart, 2008; Evans, 2002; Kulackac, Ozkan, Sucu, \& O'Lynn, 2009; Meadus, 2000; O'Lynn, 2004; Roth \& Coleman, 2008). The evidence generally supported a pattern of language neutrality in the course syllabus, text, and hand-out materials; coursework did not appear to attribute only feminine pronouns to describe a nurse; and the homework examples and case studies described nurses with both female and male pronouns. Within class, the researcher observed faculty including both masculine and feminine examples when referring to the nursing role. For example, as part of preparing the students to attend their first clinical experience at a local school, the instructor reviewed the appropriate clothing to wear to the setting. Along with indicating that women shouldn't wear open-toed sandals, the faculty also said that men could wear a tie. At another time, the researcher observed clinical faculty describing the reproductive system for both women and men, ensuring that all aspects of each were shared with the entire class.

This pattern of neutrality was further confirmed by Cliff. “I don’t feel excluded or anything and I feel like I know that Susan was more sensitive, she would definitely try and include Jack and I in things and kind of ask us questions about our perspective from 
time to time." Cliff also shared that Susan made sure to note that men and boys can also experience abuse and ensured that techniques to assess abuse for both men and women were shared as part of that curriculum section. Jack also said, "I think they treated us all neutral. We all have the ability to care for people. So I think they did a good job. I don't think they tailored it more towards women or anything like that.”

The faculty also showed an awareness of needing to use gender-inclusive language in the classroom with Marcie indicating that welcoming men into nursing was more accepted in this region due to a more liberal perspective of the people and with Polly indicating that men bring a diversity of perspective to the classroom that is important. As the program director, Vicki also shared that including male pronouns when referring to nurses is still something that doesn't always come naturally to her and that she is working on modeling this within the educational setting.

\section{Summary of Gender Neutrality}

It is interesting to note that this pattern of gender neutrality was upset somewhat when both men noted the pressure they felt to volunteer. In reviewing the data to examine how gender is described, represented, or reflected within the classroom setting, the researcher found that gender is reflected for both men and women in the educational environment. Both male and female pronouns are used to represent nurses by faculty, human anatomy is described for both sexes without assumptions that some students are already familiar with the reproductive system, and the male students did not express any concerns about the way that gender was expressed in the classroom.

\section{Kanter's Theory of Tokenism: Visibility, Polarization, and Assimilation}


This research question emerged from the literature as a theoretical perspective to describe the cause of any barriers that may be found within the educational setting. One theory about what may cause gender issues for those in the minority was proposed by Kanter (1977), whose theory of tokenism identifies three different perceptions (visibility, polarization, and assimilation) that "determine the interaction dynamics between tokens and dominants and creates the pressures dominants impose on tokens” (p. 971). The question that emerged is whether men, as gender minorities within nursing, experience visibility, polarization, and assimilation issues that impact their success in nursing school. Visibility

In this section each of these components is reviewed as case study patterns; therefore, the term visibility becomes a) visibility as gender minorities, b) visibility as pressured volunteers, c) and visibility as clinical partners. The last part of discussion inverts visibility into invisibility to faculty.

\section{Pattern 1: Visible as Gender Minorities}

Men are considered a gender minority in nursing due to their low numbers in the nursing profession. The literature has found that as members of a smaller gender group in nursing, men are not offered the same opportunities as women (Bartfay et al., 2010; Dyck et al., 2009; Kelly et al., 1996; Meadus \& Twomey, 2011; Okrainec, 1994). One theory as to why men are not as influential within the field was posed by Kanter (1977).

With the visibility perception, Kanter (1977) indicated that the dominant group has more awareness of the token in the setting and that this heightened awareness can add pressure to the recipient of that awareness. The evidence for this study indicates that 
Cliff and Jack noticed that they were only two out of 36 students in the class and that they were noticed by faculty in some ways that they didn't think that their female peers were. Cliff said he believed he was let into the program because there were not enough males. Cliff is aware that his gender minority status may be a contributing factor to his admission into the program and showed his awareness of the limited numbers of men in nursing. Jack also suggested that Cliff's physical attributes made him more visible to the class and faculty. "We were talking [in class] about abuse and some of the stereotypes that you put on people, and um, Susan, shared that she instantly guarded herself more around Cliff because he's so tall. She talked with Cliff about how his presence was a little bit overwhelming and Cliff was like, 'Oh, I’m just a gentle giant, you know.' Jack’s awareness of Cliff's height being commented on could show that he was feeling more visible in the class and that he was possibly concerned with being put on the spot as well. Cliff became more aware that his height might cause others to be intimidated.

Susan had mentioned something in class last week, and it's something I forget about. I am a tall man and I don't think that I come across as being scary or intimidating, you know? But I know some people probably feel that way just because of their past experiences or maybe something about me triggered something for them. And I don't necessarily think about that on a daily basis and Susan mentioned during a seminar that she notices when there's a large man in the room - she's like 'Cliff's a very large man and he can be intimidating to some people.' She's like 'I know he’s very friendly and he's approachable, but I notice his presence in the class.' Hearing that it kinda made me put up my radar. You gotta be conscious of how people might perceive you.

Cliff was beginning to understand that he would need to be aware of how others perceived him as part of his nursing role. However, this also seemed to be an accommodation that Cliff has to make based on his gender. Would Susan have remarked 
about feeling threatened by a tall woman? It seems unlikely and as such, Cliff's gender was visible in a way that the women in the class do not experience. Jack also mentioned being more visible as one of the few men in the class when he skipped a class during the

middle of the term. Jack noted that other women in his class were not identified as being missed but that he was. However, Jack might not have been aware that his fellow students might have communicated their absence with the instructor in advance. This fits with earlier patterns where Jack was identified as not being as communicative with his faculty as his peers were. It also highlights that Jack was not used to communicating with his faculty in a way that informed them of what he was thinking or doing related to his coursework. This could be an example of how different communication styles or expectations within the program may put Jack at a disadvantage.

\section{Pattern 2: Visibility as Pressured Volunteers}

In addition to feeling more visible than others in class, Cliff and Jack discussed examples where they appeared to feel more visible in clinical coursework. Cliff shared that he and Jack had discussed feeling pressured to volunteer as practice patients during clinical coursework. “In clinical, Jack and I both talked about this, we almost feel we're a little more obligated to volunteer.” Cliff believed that others in the classroom expected him to be the practice patient in clinical. Jack confirmed this sentiment and said that this expectation was because men can more easily expose their chests.

Ah, being the only two males we are able to show more and I guess the male stereotype of being um, you know - ah, you know less concerned about what people think about you or like you know we'll take one for the team whatever. 
Um, in terms of volunteering to be the patient upfront, I felt pressure to jump up there and - and make it so none of the girls felt uncomfortable.

Jack showed concern about the comfort level of the women in the class and felt a need to keep them from feeling uncomfortable by volunteering. However, Jack felt pressured to perform by his fellow students and seemed uncomfortable with volunteering to be a patient where his chest was exposed. Cliff and Jack believed that as men it is more socially acceptable for them to remove their shirts and bare their chests in public and they felt that there was an unwritten expectation from others in the class that he and Jack act as the example patient in class. Their heightened awareness of the comfort level for the women is another way of expressing their own discomfort in this setting.

The researcher also observed this phenomenon during direct observations of the course when a faculty member asked the class for volunteers and none of the students came forward. After a few minutes of waiting, this researcher observed a female student next to Jack who nudged him and suggested that "he go up.” After a few minutes of waiting where none of the students volunteered, another faculty member in the class volunteered to be the patient. Using students to illustrate clinical skills is a norm within the classroom and it appears that faculty ask students to demonstrate during clinical coursework which is not typical to a traditional classroom experience. This expectation for this type of in-class participation may cause discomfort for students and faculty may need to set up this expectation more clearly with students in order to get their cooperation. In addition, while Cliff and Jack felt pressured by the other students to volunteer as patients, they also believed that as men, they are more comfortable exposing 
their bare chests in a mixed class than a woman would be. However, neither felt comfortable enough to volunteer in this situation. This showed their resistance to being expected by the majority group to volunteer during class demonstrations and highlights that this type of in-class participation may cause undue discomfort for those in the minority group.

Cliff and Jack also indicated that the learning experience of pretending to be the patient is different from that of an observer. Cliff stated, “I think at times it's more embarrassing to be the patient because you kind of don’t know what to do. You know what is supposed to be happening and you know what to do but you're just kind of sitting there.” The experience as a pretend patient is an uncomfortable and less active role for Cliff. Jack also indicated that it is harder to learn the skills when volunteering to be the patient. "So I did do it once, but after I do it once, I mean, like there's some benefits to being able to sit and watch instead of being the one that goes up there.” Volunteering to be the patient takes the student outside of the learning experience because he or she is not able to see the same things that the students in the audience see. Feelings of selfconsciousness, embarrassment, and not knowing how to act may infringe on the ability of Cliff and Jack to focus on what the instructor is saying or doing when they are volunteers. In addition, asking for student volunteers to participate in class demonstrations may inadvertently cause discomfort for students in the learning environment. If it is expected that students volunteer for in-class demonstrations, it may be important for faculty to address any learning gaps that may occur for the student due to the volunteer 
participation. This is an important finding so that learning barriers are not inadvertently created by faculty because help is needed during in-class demonstrations.

\section{Pattern 3: Visibility as Clinical Partners with Female Classmates}

Another area where the male nursing students felt more visible was when practicing clinical skills on fellow female students. Cliff had a heightened sense of visibility and increased level of discomfort when working in clinical situations with his fellow female students. "It's more like an uncomfortableness when we do skills on each other. I just dread getting paired up with someone who doesn't' want me to work with them.”

Cliff was anxious that he would be partnered with another student who didn't want to work with him because he was male. He also expressed feelings of discomfort on behalf of the women in his class. "I guess I kinda feel uncomfortable sometimes working with the female students. It's not necessarily me that feels uncomfortable. I feel uncomfortable for them to have to work with me.” Cliff was acutely aware of his gender partnering with female peers when practicing clinical skills and was concerned about making the women uncomfortable. "I think it’s more uncomfortable for me when I am doing the head-to-toe exam on a fellow female student because you have to do the heart and you don't want to touch anything. And there's really no way to do what you need to do without touching their chest area. I feel uncomfortable for them.” Cliff's concern for the comfort level of female peers that partnered with him underscored his own discomfort and feeling of visibility. 
Jack also noted a level of discomfort when partnering with female peers. “And also I think that the women felt more uncomfortable practicing on...exposing themselves to us. So by default Cliff and I kind of do head-to-toe together.” Jack also showed a desire to manage these feelings of discomfort. "For head-to-toe assessment, I picked Cliff just to keep it easy for all of the females that may be uncomfortable.” Jack and Cliff picked each other as clinical partners in order to alleviate any discomfort they might have with partnering with women or that the women might have partnering with them. Both Cliff and Jack showed an acute mindfulness of their gender during clinical skills and exhibited discomfort with the need to touch sensitive areas on a female classmate's body when practicing clinical skills in class.

In addition to being aware of his gender and having to touch sensitive parts of the body when practicing clinical skills, Cliff indicates that his personal relationships with the women in the class enhanced his discomfort with practicing clinical skills with them because it was too intimate. Cliff believes it can be difficult to overcome the personal awareness of practicing skills on a peer because social customs come into play. Specifically, it may be more difficult for friends of opposite sexes to expose portions of their body with each other due to social mores that inhibit friends from invading each other’s personal space. Being required to invade a woman's personal space made Cliff feel vulnerable with peers with whom he worked hard to develop collegial relationships.

Ultimately, Cliff and Jack felt more visible when partnering with female peers and were be concerned with causing the women in the class discomfort. This underscored their own discomfort with working with their female peers and highlighted 
how social connections and mores may pose a barrier to opposite sex students pairing up to practice clinical skills on each other. Both Cliff and Jack felt more visible during the clinical setting and were more aware of their gender differences in the educational environment.

\section{Pattern 4: Visibility as Invisibility to Faculty}

The faculty were aware that there were few men in the class but beyond noting the limited number in the specific cohort, they gave little indication that they were more aware of Cliff and Jack than the other students in the class. Susan commented on this when she compared the actions of Cliff and Jack in the Nursing 210 A class to another cohort that only had one male nursing student. "They [Cliff and Jack] consistently did sit up in the front. So - you know I'm not willing to say what it means yet. But they didn't need to hide in the crowd and the other man [the male student in a different cohort] acted very differently than these two. And I can't say if that's gender related.” Susan was aware that the Nursing 210 A class had only two men in the cohort but she did not indicate that she was more aware of the men due to their limited numbers. However, Cliff and Jack talked about the importance of having another male in the class and identified that this was an important factor to their feelings of comfort. It could be that having another male to sit with in this cohort allowed both Cliff and Jack to sit at the front of the class and not feel uncomfortable by being more visible in front of their peers.

Marcie also showed an awareness of the limited numbers of men in the cohort and noted that previous cohorts have had more men. So I'm actually really disappointed, I was like two? Last winter half of my clinical group was male and I was like yes finally 
we're turning the corner here. Marcie showed support for increasing the number of men in the program and indicated previous cohorts have had a larger contingent of men. Polly also noticed the limited number of men in the cohort and said, "I mean I was absolutely surprised when we only had two. Polly also seemed to be familiar with having larger numbers of men in the program and her expectation was that there would be more men in this cohort as well. Both Marcie and Polly seemed to expect that higher numbers of men would be joining the cohort and the gender mix of each class is something that they were tracking.

Although the faculty were aware of the limited number of men in the cohort and seemed familiar with other cohorts that had men in them as well, they did not seem to view Cliff and Jack as more visible within the Nursing 210 A\&B sections than any other student and they tended to speak about their impressions of the cohort as a whole. Susan indicated, “I didn’t see that they [Cliff and Jack] interacted more or less. One thing I appreciated about the group at large is that they were very responsive and respectful.” Marcie also talked about her impressions of the class as a whole. "It feels like they as a group come in very focused. I see that with both genders.” Susan and Marcie were reluctant to identify specific differences that they might have seen with Cliff and Jack in conversation with the researcher. Polly mentions that that Cliff and Jack are more visible to her but only in a surface manner. "Yeah, I think it’s just easier to notice the males. I mean obviously, I know their names first.” Polly considered knowing the names of Cliff and Jack first as just a part of getting to know the cohort and recalling those characteristics of students that most readily help recall their names. Overall, the faculty 
were aware that there were limited numbers of men in the cohort but were unable or unwilling to identify specific differences that they noticed with Cliff and Jack in comparison to their female students. This may be in part because differences do not exist or it could indicate that the faculty are aware that the researcher is also an administrator within the school and they are conscious of having the researcher in the classroom.

Beyond an awareness of the limited numbers of men in the cohort and noting a few differences between Cliff and Jack and their peers, the faculty did not seem aware of whether they were more aware of Cliff and Jack in the classroom any more or less than any other student. However, we know from Cliff and Jack that the men felt like the faculty were more aware of them and called out their physical attributes as well as commented when they were absent. While this researcher noted that the faculty in both sections of Nursing 210 A\&B interacted with all students, providing instruction, giving opportunities to answer questions, and providing feedback as warranted it, the faculty may have been more conscious of their actions while being observed. Observations showed that Cliff and Jack did not appear to receive extra attention and they were not asked to do anything the other students were not asked to do indicating that the faculty did not treat them differently. However, the faculty and administrator do note that they are aware that men generally share less and have a harder time with mother-baby content. Therefore that fact that the faculty and administrator fail to identify these as specific differences for Cliff and Jack makes it seem as if the differences don’t exist. A more likely explanation though is that the faculty and administrator are reluctant to highlight or acknowledge any differences that they saw for Cliff and Jack and so the differences that 
do exist for Cliff and Jack within the classroom appear to be invisible to the faculty and administrator.

\section{Summary on Visibility}

Cliff and Jack did feel more visible in the classroom environment and noted when their physical characteristics like height or absence from class were noted by faculty and other students. In addition, Cliff and Jack identified feeling pressured to volunteer and felt more visible to the class and faculty to perform during in-class demonstrations. They also experienced discomfort when partnering with female peers in the class when practicing clinical skills and felt that either the women would be uncomfortable working with them or that something about the differences in gender made the interaction more difficult. Finally, the faculty were aware of the limited numbers of men and may have been more aware of the men in some ways like knowing their names, but they did not appear to notice the men any more or less than the other students and did not seem to be aware of the areas where Cliff and Jack felt more visible in class.

\section{Polarization}

Kanter (1977) defined the polarization perception as the increased awareness of tokens by dominants within the group where dominants are more aware of the differences of the token, which causes the dominants to isolate or exclude the token from social interactions that may influence the token's professional mobility and success. Within nursing education, the concept of polarization can be viewed as a heightened awareness of men by female faculty or peers by which the men are isolated and excluded from learning situations that would cause them be less academically successful. The evidence 
for the polarization perception is addressed in two patterns: 1) experiences of social exclusion and 2) gender-based exclusion.

\section{Pattern 1: Experiences of Social Exclusion}

Neither Cliff nor Jack ever identified being excluded by their faculty members and there were no instances of direct observation by the researcher where Cliff and Jack were excluded. This fits earlier patterns where Cliff and Jack described feeling connected and bonded with their faculty. However, both Cliff and Jack described making connections with women in the cohort as taking more time and Cliff in particular expressed that having experienced loneliness at the beginning of the term. "I felt lonely at first and I think that was because I didn't really know anybody in my group and there were so many women that I kinda wanted to bond with a male.” Cliff expressed feeling isolated based on his gender from the women around him and wanted to connect with another male as a point of feeling like he knew someone. Cliff encountered social isolation from the women in the class and described a scenario at lunch when he was excluded by a group of women in his class.

We were in clinical, and when I came back there were like four women that sat down at my table and I hadn't really talked with them before but my stuff was already there so I just sat down. I didn't wanna move and sit with people I knew better, but when I happened to sit down, they were all talking about the births that they had experienced and I was just sitting there with nothing to contribute to the conversation and it made me kinda clam up. I don't think they even acknowledged that I was there, and I definitely didn't feel welcomed.

Cliff felt out of place when women he didn't know sat by him at lunch and then proceeded to talk about intimate birthing experiences where he had little to contribute and 
was not invited to do so. Cliff also talked about his physical attributes as a possible explanation for why others in the class didn’t talk to him right away

I am a tall man and I don't think that I come across as being scary or intimidating, you know. But I know some people probably feel that way just because of their past experiences or maybe something about me triggered something for them.

Cliff showed an awareness of being taller than others in the class and wondered if this physical attribute was causing him to be excluded by others in the class.

Jack didn’t express loneliness directly “I was talking with some other nursing students and they asked me, like, how is it working with all these females all the time? And my thought was like, I'm okay, I'm around a bunch of females, but I guess I see them more as people.” Jack indicated that he didn’t notice gender differences in class. However, he did notice that his female peers were less likely to partner with him when practicing clinical skills. He said, "I did try to stray over and it was like hey, Bell, you want to ah just switch up a little bit. And I think Bell was still uncomfortable with her toes not being done. So she’s like oh, I have Piper, you know were going thought it.” This type of exclusion in the clinical setting by another peer could have caused Jack to lose out on practicing a critical skill in class. Had Jack not had Cliff as a back-up practice partner, this type of social exclusion during partnering exercises could have limited Jack's learning as well as impacted his feeling of welcome in the class. Luckily, Jack did have Cliff to partner with and his learning did not seem to be adversely impacted. 
Feeling lonely and being excluded from social and working groups are consistent with the polarization perception. In addition, the examples where Cliff and Jack were excluded socially and in the clinical setting were congruent with the observations of class settings. During direct observations of class, the researcher noted that at the beginning of the term Cliff and Jack sat exclusively together, did small group in the same groups, and spoke with each other almost exclusively during breaks and after class. As the term progressed, the men could be seen interacting more with the women that they sat with in the theory coursework and seemed to be mutually engaged by the women in the class as well. However, in clinical settings where the students were asked to partner up in order to practice clinical skills, the men again worked exclusively together and the women appeared reluctant to partner with either of them.

\section{Pattern 2: Gender-Based Exclusion}

Another aspect that is consistent with the polarization perception is that Cliff and Jack both anticipated not being welcomed in specific clinical settings. Cliff had already experienced being excluded from working with a female patient. "I did experience at this local hospital, there were quite a few female patients that did not want me around. It kinda hurt my feelings a little bit but at the same point I understood.” Cliff acknowledged that his feelings were hurt when a patient asked not to work with him and he also seemed to anticipate that this would happen again. "I know there will be a chance that I may not be allowed to work with females in certain scenarios. They may not want me there.” Cliff's previous experience with being excluded from working with a female patient caused feelings of isolation and he was preparing himself for this to happen again. 
Jack also showed an awareness that he would be unwelcome in some situations. "Yeah, there are gonna be a lot of settings that gender is gonna play a role like when I'm doing treatment for patients. If a young woman doesn’t feel comfortable with having a male nurse or whatever, what do you do in that situation? Hand it off and get a different patient, you know?” Although Jack didn’t give a specific example where he had been excluded from providing care, he showed an awareness that this would occur in the future and was anticipating how he would respond.

Although the faculty did not show any exclusion of the male nursing students in their teaching, they did show an awareness that men may be excluded from certain areas in the nursing profession, specifically related to mother-baby experiences. Susan stated, “The truth is they're not as welcome in maternal child work, that kind of thing. I know that when I talk about pregnancy and so on, we just get going in a way that can exclude men, and people who are not familiar around that process." Susan showed an awareness that the language used when talking about mother-baby issues may exclude men due to their lack of personal experience with birth. Marcie also mentioned that men are not welcome in mother/baby units and stated, "I have friends that are nurses who are male saying they were getting a lot of push back like no, they can’t go in and see my patient because they're a man.” Marcie’s exposure to this type of exclusion comes from talking with male nurses in the field and shows a sentiment in the practice arena that men are unwelcome in mother-baby units. As an administrator, Vicki shared a similar sentiment. "I think that absolutely in the practice world that there are some practice areas where men are less welcomed. I’ve heard people talk about men in midwifery and how that is not 
PERCEPTIONS OF MALE NURSING STUDENTS IN NURSING EDUCATION 126 necessarily something that's desirable." Vicki is also aware that men are unwelcome in mother-baby units. These faculty shared an understanding of a common norm in nursing that men may be excluded in some areas of nursing practice and particularly in motherbaby units.

The findings suggest that polarization is present for men in the clinical setting and that it is a recognized norm by both faculty as well as the students that men will not have access to certain professional fields of nursing. This is not discussed openly by faculty with the students but seems to be intuited as part of the nursing culture by the men.

\section{Summary of Polarization}

Cliff and Jack experienced polarization in the form of isolation and exclusion from the female members of their cohort when they sought to make initial social connections with female peers, to partner with female peers in the clinical setting, or when they anticipated working in specific clinical settings. While these instances did not occur at a frequency that seemed to bother Cliff or Jack or hinder their educational experience, these instances highlight how their gender influenced the way in which they experienced the educational setting.

\section{Assimilation}

In the assimilation perception, Kanter (1977) indicated that the dominant group will generalize the most visible characteristics of the token to all members of the token group. These perceptions by the dominant group lead tokens to be impacted by the cultural norms and expectations of the dominant group, and the tokens have little ability 
to affect, change, or impact the dominant culture in a way that is more inclusive towards them (Kanter, 1977).

In the analysis of the materials, two areas emerged that suggest that the dominant group of female students or faculty may be stereotyping the men in a way that was then attributed to male nurses at large. The first stereotype related to the pressure the male students expressed about volunteering to be a patient in the clinical setting. This perception by the men seemed to be attributed to the commonly held societal belief that men are more comfortable with exposing their chests in public. The second stereotype related to the shared understanding that men are not welcome in some clinical experiences and that Cliff, Jack, and the faculty all believed that they would be excluded from providing patient care to some patients.

Both of these findings have their foundation in gender stereotypes and cultural norms. It could be argued that in American society today, exposing a bare chest in public is only socially acceptable for men, potentially making it easier for students and faculty to call on the men for in-class demonstrations. However, this becomes problematic for men like Cliff and Jack because while it may be socially acceptable for men generally to bare their chests in public, it assumes that Cliff and Jack specifically are comfortable with baring their chests to their classmates. In addition, the idea that male nurses will be excluded from mother-baby units seems to have its basis in stereotypes about men not being nurturing or caring enough to work with women and children. However, many male doctors work in OBGYN units and so this stereotype within the nursing culture seems to be based on feminized norms in nursing that women only want women 
attending them during birth and when working with babies. However, in our culture today, the birthing experience has become more open and public where fathers, parents, family, and friends are invited into birthing rooms. This stereotype that male nurses are not welcome in the birthing room or with mothers and babies seems out of date with current norms within our society and seems to be a standard that is still being upheld in nursing for unknown reasons.

Cliff and Jack were resistant to being stereotyped as class volunteers, discussing the pressure they felt about this with each other, and then choosing not to volunteer as the class progressed. In this way they were employing their own coping skills against the assumption that they would always volunteer in this manner. While Cliff and Jack seemed willing to challenge the idea that they should volunteer for in-class demonstrations, they did not seem to challenge the idea that men should be welcome in mother-baby clinical units. In addition, neither the faculty nor the administrator indicated that they were actively challenging this stereotype in practice but couched this experience as something that depends on the patient. However, during member checks with the data, Marcie added more to this topic. "I do think it is wrong that men are not included in some mother-baby units and I don’t think it is an acceptable practice.” However, when I asked her how she currently challenges this stereotype, she hesitated and then indicated that she would "say something at the individual level." This shows that Marcie has some discomfort with these findings that faculty acknowledge and expect that men could be excluded from certain clinical situations. Marcie was not comfortable learning that the 
faculty may accept that men may be excluded from certain practice experiences as a standard of nursing practice that may be gender-biased.

As the gender minority, Cliff and Jack felt that a few cultural norms were generalized to them in the clinical setting. This occurred with the gender norms around baring skin on the upper body as well as around the presence of men in mother-baby units. These norms may explain how Cliff or Jack would act in particular clinical situations and why they resisted being stereotyped by refusing to volunteer to bare their chests as part of an in-class demonstration. However, they showed little resistance with challenging the idea that they would not be welcome in some clinical settings and this shows that they also may be under the influence of specific cultural norms where they anticipate feeling less comfortable as men in mother-baby units. Whether they resisted these generalizations or not, it is clear that gender stereotypes and cultural norms influence some clinical experiences for men in nursing.

\section{Conclusion of Kanter's (1977) Perceptions of Tokenism}

While traits of visibility, polarization, and assimilation appeared to exist for Cliff and Jack in this course, what is most important to consider is how these occurrences impacted their educational environment, academic success, and retention. The evidence shows that Cliff and Jack experienced feeling more visible, isolated, or stereotyped in the educational setting and were more aware of these occurrences than their faculty. However, these examples did not occur with enough frequency or intensity to hinder Cliff and Jacks academic success in Nursing 210 A\&B or continued progression in the nursing program. Indeed, Cliff and Jack minimized the role of gender in their educational 
experience and showed resistance to being expected to participate in a way that they felt uncomfortable with. This shows a level of resilience to not be type cast in a certain role, which could be a useful tool as they progress in the program.

\section{Faculty Aware of Gender Barriers in This "Estrogen-Soaked" Field}

A body of literature exists that identifies barriers that may exist for men in nursing and provides recommendations for faculty to address gender barriers in the educational setting (Anthony, 2004; Bartfay et al., 2010; Bell-Scriber, 2008; Dyck et al., 2009; Kelly et al., 1996; Meadus \& Twomey, 2011; Okrainec, 1994; O’Lynn, 2004; Poliafico, 1998; Yang et al., 2003). Among these were recommendations to help faculty be aware of and help create a welcoming and supportive environment for male nursing students (Anthony, 2004; Bartfay et al., 2010; Bell-Scriber, 2008; Meadus \& Twomey, 2011; Okrainec, 1994; O’Lynn, 2004). However, little research shows whether faculty members are aware of the barriers that men in nursing may experience or whether faculty are making any efforts to address these barriers within their educational setting. This study sought to identify to what extent the faculty were aware of barriers mentioned in the literature that may exist for men in nursing, if they were aware of specific barriers faced by men in their nursing classes, and if they were instituting any interventions to address or minimize barriers that may exist in an effort to create an inclusive learning environment for men.

The evidence from this study shows that Susan, Marcie, Polly, and Vicki all readily expressed a familiarity of some barriers for men in nursing. This included an awareness that nursing is a female-dominated field, knowledge that men are underrepresented in nursing, mindfulness around using both female and male pronouns 
when referencing nurses, and an understanding that men are still excluded from some clinical areas in nursing practice. Susan, Polly, and Vicki showed their awareness that nursing is a female-dominated field through their comments. Susan remarked that "the field is very estrogen soaked” indicating an awareness of the feminization of nursing that continues. Polly also stated, "Men have to come to terms with being a male and coming into a woman-dominated profession.” Polly believed that men have already mentally prepared for joining a female-dominated environment prior to applying to nursing school. Vicki also indicated an awareness of the feminization of nursing and the barriers that this may cause for male nursing students when she said, "I am acutely aware as an educator and an administrator that men are entering a world that is so predominantly female. And the workplace is shaped by the feminine perspective.” Vicki was aware that the educational environment had been shaped by women; however, she was silent on how to address any issues that this may cause for men.

Susan showed an understanding that men are underrepresented in nursing. "I believe that they're marginalized. I believe that they try to make us happy, and I think that there are ways that we could change our curriculum that would help integrate them more effectively, and that would be having more men teaching and lots of things.” Susan was not only aware that nursing education is dominated by a feminine view but indicated that having more male faculty may serve as a role model for helping men feel more included within nursing. In addition Susan, Marcie, and Vicki showed that their own behavior in the classroom could be a limiting factor for men in nursing and noted the importance of using gender-neutral language in the classroom when referring to nurses. 
Susan said, "I know that when I talk about pregnancy and so on, we just get going in a way that can exclude men, and people who are not familiar around that process.” She indicated that men may find it harder to be included in conversations around pregnancy because they don’t have their own personal history or experience to bring into the conversation or they may just not have had as much exposure to the topic as female students, which may put them at a disadvantage for participating in the conversation. Marcie and Vicki also showed an awareness of how the use of gender-specific pronouns to refer to a nurse or a doctor can become labels that help enforce stereotypes. Marcie stated, "I think our use of language is very important. To be aware of not always saying every nurse is a 'she' and every physician not being 'he'.” She also said “I’ve tried to be really good about making nurses men when I do a scenario or use both genders. But I think some people always refer to nurses as 'she'.” Marcie was conscious of using both female and male pronouns to describe nurses in the classroom as a way to reflect that nurses can be of both genders and to be more inclusive in her representation of nurses as a whole. Vicki said, "I think that that happens very subtly and I think we all need to be really, really careful about the languaging that we use and that kind of thing so that we are inclusive and that our expectations are geared truly toward what our expectations are of nurses, not necessarily of what our expectations are of female nurses.” Vicki was aware that the way that language is used in the classroom can help establish cultural norms that may be gender-specific and has the potential of excluding some groups. 
In addition, the faculty were aware that men were underrepresented in nursing. Marcie indicated that she was aware that there is not gender equity in the program. Polly stated, “I was absolutely surprised when we only had two," indicating that she was expecting more men in the cohort.

Susan, Marcie, and Vicki also noted that men may be unwelcome in some clinical areas like mother-baby units. Susan stated, “And the truth is they're not as welcome in maternal child work, that kind of thing, indicating that men have fewer opportunities in sites in mother-baby units. Vicki also stated, "So I think that absolutely in the practice world that there are some barriers to... there are some practice areas where men are less welcomed. Vicki was aware that some clinical arenas will not allow men to practice. Marcie shared a story about another female nurse that she was working with in the hospital who told her, "You know this was the last thing that women had a corner on. Men already took over education and now look now we're getting all these men in leadership positions in nursing. This is the last thing that women had.” Marcie shared this story as an example of that idea among some practicing nurses that men are not welcome in nursing and are perceived as taking opportunities away from women within the profession.

These examples show awareness by the faculty and administrator of some of the barriers that men may face in nursing education and within practice. However, none of the faculty identified any specific barriers that may have been encountered by Cliff and Jack in the Nursing 210 A\&B classes. In addition, there was no indication that the faculty or administrator had any specific support structures or activities within the class 
or curriculum to address or minimize barriers that may have existed for Cliff and Jack. When asked specifically about how they might prepare men for being excluded from clinical sites, Polly said, "As far as directly in the curriculum addressing it, I think it would be probably more of in a clinical, in a debrief.” Polly saw being excluded from a clinical experience or patient setting as something that occurs on the individual level and that can be reviewed after it occurs. She did not seem to see this experience as something that could happen to men and women and was not something that she was preparing students for in advance in a general manner. When Vicki was asked about the awareness of faculty as a whole to prepare students for being excluded from specific clinical situations, she also talked about addressing this in a case-by-case way.

When I taught in mother-baby and had men on the unit, I would specifically take them aside and talk to them about their feelings because they weren't always selfselecting to be in postpartum. They were sometimes thrown in there and so I talked to them about their comfort level and I talked to them about the possibility that a patient might refuse them as a nurse because they were a man. And then of course, we have cultures, like women from Muslim cultures and care by a man is forbidden by their culture. So I always try to do that personally. I'm not sure how other faculties are handling that.

As a nurse who had worked on the mother-baby unit, Vicki was aware that Cliff and Jack might be excluded from this type of clinical situation because of patient discomfort or religious or cultural background. She showed an awareness about working with individual male students within that unit to prepare them for the possibility that they would be excluded from providing care and assessing their comfort level with motherbaby nursing practice. However, as the program director for the undergraduate program, Vicki was not aware of any concerted efforts by the faculty to prepare students more globally in the curriculum for this type of exclusion within the educational setting. In 
addition, she did not indicate an awareness of any faculty trainings or discussions about the types of barriers that men might experience in class or how to help address those barriers in the educational setting. Finally, neither the faculty nor the administrator indicated any knowledge of or plans to assess the male nursing student experience, beyond evaluating individual student academic success, to identify what, if anything, the male nursing students might have needed in order to be successful.

Despite this lack of awareness of specific barriers, the faculty and program director did express a desire to have men in the nursing program at PNMU with Vicki saying, "I really think that men in nursing add a huge richness to our profession and I think that they are an important subgroup of our profession.” Polly also stated that having more men in the program would "shake us up too, indicating that she was aware that men bring a different perspective to nursing that is valuable. "Shake us up" is an important phrase here as Polly seemed to acknowledge that these differences will perpetuate conflicts that will need resolution.

The assumption of gender neutrality was reinforced by Marcie, who said: "I’ve seen people make assumptions about male nurses and I'm like whoa. You need to think of him [the student] the same as every other nursing student.” Vicki also expressed similar sentiments. "I think we really need to watch what our expectations are and that we really need to make sure that we're talking about professional expectations for all students.” Marcie and Vicki showed an awareness and willingness to treat students similarly and indicated their knowledge that this is an important step towards creating a welcoming environment for men in nursing. 
The evidence suggests that faculty are sensitive to creating an inclusive environment for men in the classroom by using inclusive language and examples. In addition, there seemed to be a willingness to welcome men into the educational community. While the faculty did use gender-inclusive language in teaching situations, they did not seem to be aware of specific barriers that Cliff and Jack might have encountered, like their heightened discomfort in the clinical setting when working with female peers or feeling pressured to volunteer. None of the faculty identified additional barriers that the men in Nursing 210 might have specifically encountered beyond identifying specific professional areas where they were not welcome to practice. When the faculty were asked specifically if the students received additional preparation about how to deal with being excluded from delivering patient care to a female patient, they talked about handling that on an individual level but none expressed a collective attempt to include this more globally in the curriculum or expressed a desire or need to have a conversation about this at as a faculty group.

The faculty and the administrator all appeared to be aware of gender bias in the greater nursing profession and expressed a desire to treat the men similarly to the other students. They identified language as a potential barrier and talked about using genderinclusive language as a way to engage with the male students in the classroom in the same was as with the females. However, the faculty and administrator did not specify how having Cliff and Jack in the classroom made the classroom more diverse in thought or practice and they did not share any knowledge that Cliff and Jack might have experienced barriers within the Nursing 210 A\&B course. While Cliff and Jack 
appeared to be treated equally in the classroom, the faculty and administrator did not provide additional support to the male students specifically and had limited knowledge of additional barriers, beyond using inclusive language, for men in the educational setting.

\section{Conclusion}

When reviewing the evidence related to the questions of 1) understanding the nature of the interactions between nursing faculty and male nursing students in the educational setting; 2) examining how gender is described, represented. or reflected within the classroom setting; 3) reflecting the perceptions of visibility, polarization, and assimilation identified in Kanter's (1977) theoretical framework of tokenism; and (4) identifying to what extent faculty are aware of gender barriers in the classroom, a pattern emerged showing that the faculty and administrator had an awareness of barriers that men might experience generally within nursing, but not a specific understanding of what barriers might exist within the educational climate at PNMU. In addition, while some interactions were found to be uncomfortable for Cliff and Jack during Nursing 210 A\&B, as in when they partnered with female peers in clinical or felt pressured to volunteer, this discomfort did not seem to impact their educational success.

In fact, Cliff and Jack were aware that they were joining a female-dominated profession and didn't seem to be concerned about how that dominance might impact them. Cliff said, “I think that gender plays a very minimal role but ultimately it's how the individual is and what their strengths are that determine if they're gonna succeed in nursing or not.” Cliff believed that the individual's ability to perform as a nurse will outweigh any gender barriers that he might encounter. He also said, "I think that it 
doesn't matter what your gender is. We can all do the same job; I think women are definitely given that role of being the more sensitive and more nurturing but that doesn't mean that men can't be that way either.” Cliff seemed willing to challenge stereotypes that only women make good nurses. Jack said, "I think, when you think of a nurse, you think of a woman. You think of someone being motherly. Hopefully that's changing.” Jack saw the field as being more open and accepting for men and was anticipating that the feminization of nursing was changing to be more equitable. 


\section{CHAPTER VI: DISCUSSION AND IMPLICATIONS}

For this single instrumental within site case study (Stake, 1995), how men in the Nursing 210 A\&B course experienced the educational environment was explored. The findings were reported first in Chapter IV using the original theoretical propositions that framed this study in Chapter I. Next, the analytic technique of pattern matching (Yin, 2009) was used in Chapter V to compare expected patterns relating to the study’s four research questions along with alternative explanations. In both levels of analysis, the findings showed that the two male students felt connected and supported by their faculty, that male gender played a noticeable role in the classroom, and that Cliff, Jack, and their faculty agreed that the two men were less comfortable with mother-baby course content and agreed that the two men could expect to be unwelcome in some clinical settings. In addition to these findings, some important differences were also identified and are discussed in detail below.

\section{Discussion of Theoretical Propositions from Chapter IV}

In Chapter IV, the evidence from the case study was compared to the original theoretical propositions: 1) men are isolated from faculty and peers in nursing school, (2) men experience barriers in the educational environment, and (3) as gender minorities, men may not be as academically successful in a female-dominated educational environment. For each of these propositions, key findings are discussed below. 


\section{Men Were Not Isolated from Faculty and Peers in Nursing School}

Cliff and Jack generally felt connected with their faculty and grew their connections with female students over the course of the term although this was more difficult for Cliff at first. This contrasts with the literature that indicated that men will be isolated and excluded from social relationships that are important for learning in nursing school (Anthony, 2006; Denhart, 2008; Evans, 2002; Kulackac, Ozkan, Sucu, \& O'Lynn, 2009; Meadus, 2000; O'Lynn, 2004; Roth \& Coleman, 2008). In a more limited way, Jack felt excluded from female classmates, but he also showed more reticence to engage with female faculty which posed an instructional challenge for the instructors. The data showed that Cliff and Jack's successful immersion into the class and learning community began with their connection with each other. In fact it was clear that the most significant relationship that Cliff and Jack established in class was with each other. Throughout their interviews, Cliff and Jack highlighted the importance of having another male in the program to work with and their connection with each other was visible to both the researcher and the faculty. Having another male in the cohort was something that both men were looking for from the first day and once that connection was established, their relationship appeared to form a foundation from which they could continue to make additional connections with others. In addition, both Cliff and Jack provided examples of how having another male student in the class helped ease uncomfortable in-class experiences, particularly during clinical coursework.

Another significant finding was that while the men eventually established friendly relationships with their female peers, both Cliff and Jack had more difficulty working 
with female peers during clinical coursework. Female students were less likely to partner with Cliff and Jack to practice clinical skills, and both men identified feelings of discomfort with having to partner with women in situations where touching sensitive areas of each other's bodies occurred. Nursing 210 A\&B coursework used a number of group work opportunities and students were expected practice certain skills with each other as well as volunteer as examples during in-class activities. This expectation that the students work comfortably with each other meant that the ability of Cliff and Jack to create relationships with female peers was important to their overall learning experience. The fact that establishing closer relationships with the women in the class took more time and created a level of discomfort in the clinical setting highlights the fact that clinical work may be an area where men are disenfranchised within the nursing educational experience. This is an area for further research.

Finally, findings showed that the female faculty categorized or described Cliff and Jack based on their communication styles in class and assessed their learning based on inclass interactions and in-class participation. The faculty found it easier to identify how Cliff was progressing as he communicated more actively with them during class and would ask for feedback. Jack was much less forthcoming in class and had a harder time making eye contact and speaking to others unless he was directly spoken to. Because of this, the faculty found it more difficult to assess his learning and Jack developed sensitivity around being a male in a female dominated environment whenever he felt singled out or put on the spot as when the instructor commented that he had missed class or when his classmate urged him to volunteer as the patient. However, Jack seemed to be 
unaware that his reticence and lack of communication with faculty impacted faculty perceptions about his learning, and Polly particularly sought Jack out to give him opportunities to speak up so that she could better gauge his understanding of the content. This in-class faculty-student communication seems to be an important aspect of how the faculty assessed student learning and was an important factor in how the faculty perceived Cliff and Jack. While Cliff seemed to naturally understand how to communicate with the faculty, this appeared to be less natural for Jack and could have been a complicating factor in his academic success.

In this study it appears that feminized class norms about frequent and open communication may show an unconscious bias in favor of female students. The literature has shown that there are communication differences between men and women in education and healthcare settings (Brown et al., 2011; Dyck et al., 2009; James \& Cinelli, 2003; MacWilliams, Schmidt, \& Bleich, 2013; Roter, Lipkin, \& Korsgard, 1991; Street, 2002) and that communication is different between men and women when one group is in the majority and has more perceived power (Fairhurst \& Snavely, 1983b). Specifically in a literature review by MacWilliams et al. (2013), a failure to identify different communication styles in nursing curriculum was identified as a barrier for men in nursing, and Dyck et al., (2009) reported that "men perceived an overemphasis on emotion in the nursing curricula and described instructor expectations for an inordinately high level or personal reflection, emotional expression, and introspection” (p. 652). In addition, Roter et al. (1991) examined communication differences between male and female physicians and found that female physicians "conducted longer medical visits, 
with substantially more talk” (p. 1083). This may suggest that differences in communication styles between men and women may be a factor in the educational environment that impacts the student experience. Indeed, in a female dominated learning environment like nursing, there may be an unconscious expectation by the faculty that men would communicate more often and with more detail in class which may pose a barrier for men or quieter female students. This may be a subtle cue that certain ways of communication are valued over others during in-class interactions and may be an important difference that will have an impact on faculty-student interactions. This too warrants further exploration.

\section{Men Experience Some Barriers}

In regard to the proposition that men experience barriers in the educational environment, the literature highlighted issues of social isolation, gender stereotypes, and expectations for men to norm to feminized standards of practicing nursing. The findings from this study show that the male gender was represented and reflected by faculty in the classroom as well as within examples and language used in the course content. While both Cliff and Jack encountered the stereotype of being assumed to be a doctor while in the clinical setting and both expressed a lack of prior knowledge of mother-baby content, neither Cliff nor Jack identified these issues as barriers to their academic success and they both minimized gender as a factor in their failure or success.

The faculty showed an awareness and sensitivity towards welcoming men into the nursing program and were aware of the need to use both male and female gender pronouns in the course content and with examples. Cliff, Jack, the faculty, and the 
program administrator acknowledged that the men had less familiarity with mother-baby content and shared the understanding that male nurses are unwelcome in some clinical settings. Although all of the participants indicated that males may be excluded from certain clinical situations, neither the students nor the faculty nor the administrator indicated that this was a situation that would be challenged, and they did not discuss a plan to proactively prepare Cliff and Jack or other males for how to handle this type of situation during the initial interviews. From these findings it appeared clear that Cliff and Jack were already beginning to accept this form of exclusion as part of their nursing role. Additionally, the idea of challenging these occurrences only came out later during member checking where a few of the faculty mentioned that men should not be automatically excluded from certain clinical sites and that this situation should be challenged when it occurs. This indicates that challenging this shared understanding of the practice setting was not automatic for the faculty but when this potential bias was brought up as an area for additional review, the faculty showed an interest in taking more direct action to challenge this norm. In addition, the literature did not appear to identify unfamiliarity with mother-baby content or being excluded from specific clinical situations and barriers for men within nursing education. For Cliff and Jack, these barriers were much more prevalent than the items identified in the literature related to social isolation and gender stereotyping. From these findings, it is clear that barriers do continue to exist for men within the educational setting but that they are shifting as more awareness of men in nursing is growing. This shift shows that continued diligence in 
understanding when and where barriers exist for men in nursing can help build awareness of where potential changes can be made to reduce areas where gender bias still exists.

\section{The Spotlight and Academic Success}

With the theoretical assumption that men may not be as academically successful in a female-dominated educational environment, the literature identified that men may be more visible and less welcome within the nursing field and that nursing faculty might be potential barriers to academic success by holding men to feminized standards for practicing nursing. The findings from this study did show that both Cliff and Jack felt that they were more noticeable in the educational setting. This was evident when the faculty and students commented on why Jack was missing from the class as well as having Cliff's height called out during an in-class example. Ultimately, this type of increased attention did not appear to hinder the academic success of the men. In fact, Jack noted that this additional interest spurred him to stay engaged and do well. However, Cliff and Jack did believe that their gender was more noticeable in uncomfortable ways when student volunteers were requested as practice patients and as a result they both began to noticeably disengage from faculty and classmates when volunteers were called for. In short, this kind of disengagement was a subtle rebellion or form of resistance.

This finding that Cliff and Jack began to turn down in-class opportunities to participate due to feeling pressured to perform has important implications for understanding the educational environment for men in nursing. As gender minorities, men may feel undue pressure to perform in class and may in turn feel more visible or singled out, which may hinder their in-class participation. If students are then evaluated 
on their level of in-class participation, a gap may occur between faculty and students about what is occurring in the learning environment that could impact student success. It is unclear if this reticence to participate in these situations affected the academic success for Cliff and Jack but it is an interesting line or inquiry that emerged that merits further research.

In addition, the evidence did not support the finding that the faculty held Cliff and Jack to a feminized standard for nursing practice. Cliff and Jack felt supported by their faculty, identified them as resources, and did not believe that they were treated differently than their female peers. In addition, the faculty made sure to use gender inclusive language in the classroom and acknowledged that Cliff and Jack had a steeper learning curve with mother-baby content but did not appear to let this lack of familiarity affect their academic success. This finding does support the notion that faculty play an academic role with student success but as well as provide support for students which can mitigate barriers that may otherwise impact success.

\section{Summary of Theoretical Propositions from Chapter IV}

Taken together, the findings show that the evidence met the theoretical assumptions in some ways but established alternate explanations in others. Cliff and Jack perceived their experience in Nursing 210 A\&B in mostly positive ways and they did not identify being isolated from faculty or excluded due to lack of gender examples, as stated in the theoretical assumptions. The feminized nursing education environment caused Cliff and Jack to connect with each other in an immediate way. They identified a measure of discomfort with partnering with female peers in clinical and felt pressured to 
volunteer in certain clinical situations. They were less comfortable with mother-baby content and developed an understanding that they would be excluded from some clinical sites. While Cliff and Jack did not identify these as barriers to their academic success and while they both tended to minimize the role of gender in the academic setting, this researcher's analysis exposed the ways in which gender could be a limiting factor in their educational experience going forward. For example, the faculty showed a general awareness of barriers for men in nursing but they could not identify specific barriers that Cliff and Jack encountered. One of these barriers was the normative use of frequent and open communication styles that held Jack at a disadvantage and in fact helped define him as an instructional challenge. While faculty noted that Cliff and Jack were often seen together working in clinical more often than with female peers, sharing fewer personal stories, or having less familiarity with mother-baby content, they did not appear to be unduly concerned about these differences and did not indicate that these differences would hinder Cliff and Jack's academic progression. Finally, the faculty shared their values for having men in the nursing program and took steps to use gender-inclusive language in the classroom.

\section{Discussion of Research Questions from Chapter V}

In Chapter $\mathrm{V}$, the evidence from the case was compared to the four research questions: 1) understanding the nature of the interactions between nursing faculty and male nursing students in the educational setting; 2) examining how gender is described, represented, or reflected within the classroom setting; 3) reflecting the perceptions of visibility, polarization, and assimilation identified in Kanter's (1977) theoretical 
framework of tokenism; and 4) identifying to what extent faculty are aware of gender barriers in the classroom. These were the basis for the protocol for the study.

\section{Faculty-Student Interactions}

Cliff and Jack identified faculty members as a resource and observed that the faculty gave them the same opportunities to learn and be academically successful as all others. The faculty described their interactions with Cliff and Jack in general terms and noted communication differences between the men with Cliff being more proactive about asking for feedback and talking more often with others in class than Jack.

\section{Gender}

The findings related to how gender was described, represented, or reflected within the classroom setting showed that male gender was made conspicuous in a feminized nursing school environment. Cliff and Jack claimed that faculty did not treat them differently nor did they perceive that they had a different educational experience than their female classmates. However, while the men were able to achieve academic success, there is some consistency with what the literature says about male isolation and dominant femininities within the nursing classroom environment (Anthony, 2006; Denhart, 2008; Evans, 2002; Kulackac, Ozkan, Sucu, \& O'Lynn, 2009; Meadus, 2000; O'Lynn, 2004; Roth \& Coleman, 2008). In several instances the men noted feeling uncomfortable in the learning environment and either adapted or coped in these situations. That both men ultimately experienced success in the nursing 210 A\& B course could indicate that the PNMU is unique in providing a welcoming educational environment for men, or it could indicate that Cliff and Jack were somehow resilient in the face of any isolation or 
dominant femininities that they experienced. Further, it may indicate that the faculty proactively engaged with Cliff and Jack to make them feel included and or welcomed in the classroom. Although the reason why Cliff and Jack did not experience the educational environment in the ways that have been identified in the literature are unknown, it does not mean that their male gender was not conspicuous and of significance in the learning environment.

\section{Tokenism and Faculty Awareness of Gender Barriers}

Key findings were identified for the research questions of the presence of tokenism as well as the extent to whether faculty members were aware of gender barriers within nursing education. Some examples of tokenism were found with the perceptions of visibility, polarization, and assimilation identified by Cliff and Jack in the Nursing 210 A\&B course. Specifically, the two men felt more visible in key situations in the classroom, including when they missed class, were engaged in topics related to motherbaby content, or were in clinical situations where they had to practice clinical skills or partner with a female peer. The men also experienced a few instances of polarization where they were excluded from conversations with peers and anticipated being excluded from specific clinical situations. Finally, the men experienced the assimilation perception when they were assumed to be doctors in the clinical setting or were perceived as being more comfortable with exposing their chests during practice clinical exams.

While these perceptions did not hinder Cliff and Jack's educational success in this class, the fact that they began to encounter these perceptions in their first term during their first nursing theory and clinical course indicates that tokenism may be a factor in the 
educational setting for male gender minorities. This is an important finding because Kanter's (1977) theory of tokenism suggests that gender differences do impact the ability of members of the minority group to advance beyond a certain level that is predetermined by the majority group. It could be that the perceptions of tokenism found in the educational setting for Cliff and Jack will grow over time or that Cliff and Jack were being sent subtle cues about how to internalize the nursing role that is based on a feminized view instead of one more suited to their individual needs or interests. In addition, it is important to note that in some instances where Cliff and Jack felt more visible, they responded by being more engaged in the classroom, but that when they felt pressured to volunteer in the clinical setting, they became disengaged. Further research on this finding could yield new strategies for how faculty engage students in theory versus clinical courses and may help students and faculty to challenge remaining stereotypes about men being unwelcome in certain clinical settings.

\section{Faculty Awareness of Gender Barriers}

The final research question related to the extent to which faculty and administrators are aware of gender barriers in the classroom. A gap exists in the literature about whether or not nursing faculty are actually aware of the current research on this topic or whether any of the recommended tactics had been implemented in the educational setting to see how they may alleviate the barriers that men may face. This study showed that faculty members and administrator were aware of the need to use gender-inclusive language in the curriculum, course content, and examples in the classroom and with students and showed that they did so. In addition, the faculty 
members and administrator were aware that nursing is a female-dominated field, that nursing education can be considered female-centric, and that this more feminized perspective may cause discomfort for men in the nursing program. They also shared awareness that men are not welcome in some clinical settings. However, the faculty and administrator did not indicate that they were aware of any specific barriers that Cliff and Jack might have experienced in Nursing 210 A\&B and did not identify many common barriers identified in the literature like social isolation, heightened perceptions of visibility, feminized nursing practice, or role strain as potential barriers for men. This indicates that the faculty may not be aware of the body of literature and series of recommendations that currently exists that highlights specific ways in which faculty can create inclusive learning environments for men in nursing. This is a key finding because the literature suggests that faculty need to do more to ensure that gender bias is not present within nursing education. If faculty are not aware of barriers that men may be experiencing within their specific classrooms, greater awareness on the part of faculty may well be needed.

In addition, neither the faculty nor the administrator indicated that a systematic or proactive approach for addressing the exclusion of students from certain clinical situations was occurring. This is a valuable finding because men may need more preparation for going into certain clinical sites than their female peers or they may need tailored instruction to prepare them for this type of experience. In addition, they may need more tools to help them cope or adapt to instances of exclusion within the clinical setting. Finally, male nursing students may need more help in learning how to advocate 
for themselves in situations where they are experiencing barriers and they may need support with challenging existing stereotypes that limit their role. Exploring this issue further may provide opportunities for faculty to model appropriate ways for male nursing students to manage this type of role conflict.

\section{Recommendations}

This research explored the educational environment for two men in a nursing program where all other peers and faculty members were women. Recommendations for male nursing students and for faculty are discussed below.

\section{Placing Students Together}

The positive role of having another male in the class cannot be underestimated in helping establish a foundation from which Cliff and Jack were successful in the Nursing 210 A\&B course and in their positive view of their faculty and fellow students. Given the structure of the Nursing 210 A\&B course where students are expected to work together, practice certain clinical skills with each other, and volunteer as examples for inclass learning, having someone with a similar perspective was clearly critical for Cliff and Jack's experience. This positive impact of having at least two men in the cohort indicates that it is important for administrators in the nursing school to be aware that the gender mix of the cohort needs to include more than just one male whenever possible. Being conscious that students seek support from those of the same gender and that men may engage each other as a way of reducing their perceptions of heightened visibility and pressure in the classroom shows that educators must be mindful of constructing nursing 
cohorts and clinical groups in a way where men can engage each other. This also means that men must be admitted to nursing programs in higher numbers. One way to achieve increased gender diversity in nursing schools is for nursing administrators to update their admission criteria to reflect gender as a form of diversity that is valued within nursing education and to identify gender diversity as a factor that is considered as important in the admissions selection process. While this value would need to be established within the current national and state laws and institutional policies for each institution, specifically identifying men as gender minorities and including that type of language as part of an institutional definition of diversity brings visibility to this underrepresented group. In addition, this sends a message to faculty and students alike that men are welcome in nursing and that their perspective and presences is valued.

\section{Breaking Down Real and Perceived Barriers between Students}

Both Cliff and Jack talked about their interest in making a connection with each other immediately on the first day of orientation indicating that establishing initial social groups is a key dynamic within the educational setting. In addition, both men indicated that establishing relationships with the women in their cohort took more time and that they encountered some barriers to growing these connections in and out of class. This has important implications for nursing faculty and administrators who need to be conscious of the importance of providing in-class opportunities for students to establish social connections. In addition, Cliff and Jack indicated that it was easier to engage with their female peers in small group settings. This leads to the recommendation that faculty structure opportunities during new student orientation and during the first few weeks of 
the program for male and female students to work in small groups together in order to help break down real or perceived barriers between students as soon as possible. It is also recommended that faculty and administrators structure time during new student orientation or class to address biases that are common in society today about nurses and to use this type of activity to discuss the value of gender diversity within the nursing profession. By creating facilitated opportunities to help students get to know each other during new student orientation and at the beginning of the term, faculty and administrators can help students create a support network that will assist all of the students' as they progress in the curriculum. This also allows the faculty to address stereotypes for gender minorities in the classroom head on as well as provides opportunities for faculty to normalize the presence of men in the classroom for all students.

\section{Reducing Social Barriers with Clinical Partners}

Cliff and Jack experienced discomfort and difficulty with finding female partners to practice clinical skills with and expressed concern about being the cause of discomfort for women when they were partnered with them. A recommendation from this finding is for clinical faculty to help remove these potential barriers within the clinical setting by preparing students in advance for how to deal with discomfort when practicing skills with peers. Acknowledging the discomfort of practicing clinical skills with peers and discussing this issue with the class as a whole to set expectations and identify personal boundaries may help reduce social barriers in the classroom. In addition, faculty should consider structuring peer-to-peer practice sessions by pre-identifying students to work 
with each other. This removes the social ambiguity of having students search to find another student to partner with, increases the likelihood that students will work with those they don't know, and gives all students a chance to be matched with students of different genders without bias.

\section{Reducing Social Barriers with Demonstrations}

Cliff and Jack identified feelings of heightened visibility and pressure when volunteers were needed for in-class demonstrations. Faculty should be aware that as gender minorities, men may feel more visible and more pressured by peers or instructors during class to volunteer, and faculty should seek to minimize instances where male students are called upon to volunteer over others. Specifically in situations where students are being asked to volunteer for front-of-class demonstrations and possibly be in a situation where they are exposing skin, it is recommended that faculty pre-assign this role so that all students get the opportunity to participate in this way. Pre-assigning frontof-class demonstrations with the designated students ahead of time also gives the students the opportunity to mentally prepare for this type of participation in advance and possibly increase their participation or readiness for this activity. Another option would be for faculty to ask for volunteers from individual's outside-of-class to participate as the patient during sensitive demonstrations so that students are not placed in a situation where they are uncomfortable and not in a position to learn along with the rest of the class. 


\section{The Importance of Faculty-Student Communication}

In-class communication emerged as an important variable in how faculty were assessing student learning. This could be a unique aspect of the Nursing 210 A\&B coursework or it could be a standard expectation of students throughout the nursing program. Regardless, it is clear that the faculty had more difficulty assessing Jack's learning because he spoke less often in class, volunteered his opinions less, and shared few of his personal stories. This indicated that Jack might not understand the culture of communication in the nursing program and might not be aware that his less frequent communication with his faculty and fellow students in class might be a limitation to his academic success. Faculty should be aware that more frequent, direct, and more personal communication with other faculty and students during class may be an expectation that is founded in a feminized view of nursing. Expectations for this form of communication could be part of the unwritten norms within the classroom that faculty use to assess whether students and men in particular, are appropriately reflecting the nursing role. If certain communication styles are identified as preferable as part of the required learning for the nursing coursework, it is important that this be named as a core component for the course and not be veiled as an unspoken classroom expectation. In order to ensure that both men and women are held to similar standards for the purposes of assessing learning during in-class faculty-student interactions, it could be helpful for faculty to more clearly outline their expectations around communication and to review with students like Jack where their communication style may need improvement. 


\section{Faculty Awareness of Educational Barriers for Men in Nursing}

While the faculty and administrator at the PNMU expressed an openness to having men in the program, were interested in knowing about potential barriers faced by Cliff and Jack in Nursing 210 A\&B, and were mindful in using gender inclusive language in the classroom, these efforts seemed to be based on the individual values of the faculty and administrator and were not discussed as part of a broader awareness held by the faculty and administration at PNMU. Indeed the faculty and administrator shared an awareness and acceptance that men are not welcome in some clinical settings but they did not identify shared tools or strategies that they use to help prepare students for this type of exclusion nor did they show an awareness that they should or could challenge this type of barrier directly within the clinical setting. In addition, the faculty and administrator did not indicate that there were trainings or discussions with the faculty as a group to increase the faculty awareness of barriers that men may experience in the educational setting and to prepare the faculty with potential interventions that they could use to help minimize barriers that may exist.

Trainings and group discussions need to be incorporated into the faculty development goals for the nursing program in order to ensure that faculty and administrators are aware of barriers that may exist for men in nursing and to develop shared tools that faculty can use to minimize barriers that may exist. In addition, more research is needed to test the effectiveness of potential interventions that faculty may use to assess their role in limiting or minimizing gender barriers within the classroom. 


\section{Implications for Future Research}

The findings from this study provided insight into when and what type of barriers two men encountered in a PNMU nursing program, adding new depth to our understanding of the educational experience for men in nursing. Additional questions arose from this work which has implications for future research. Within this study it was identified that men had a harder time establishing relationships with their female peers and that instances of social isolation, visibility, and pressure emerged from interactions with female peers. Further research is needed in this area to further our understanding of how social interactions with female peers impact the academic success for men. In addition, the men in this study perceived more barriers while in the clinical environment and more exploration of what is happening for men in the clinical learning environment would continue to further our knowledge of the barriers that men face in nursing. Finally, the faculty in this case study were not found to have a robust knowledge of the current literature related to the issues that men in nursing encounter nor did they have an established or cohesive plan for addressing barriers that they may have seen or could anticipate seeing for their male students. More research is needed in this area to identify what factors are hindering faculty in understanding these barriers and in taking action on them when they see them occur within the educational setting.

\section{Limitations}

There are a number of limitations to this study including the low number of men in the Nursing 210 A\&B cohort who were available to participate in the study, the fact that data collection could not be started until the third week of the term, and the fact that 
all participants were fully aware of the topic being studied, which may have affected the way that they behaved in class or responded during interviews. Faculty in particular were reticent to share specific details about any differences they saw between Cliff and Jack and the other women in the class, and they did not discuss specific details about how Cliff and Jack learned or handled the course content. The faculty tended to speak generally about their knowledge of men and kept their comments about Cliff and Jack to neutral or positive remarks. This is a limitation because this study was looking at the perceptions that faculty have about men in their class, and these faculty may have withheld information that would have provided further clarification on this topic. In addition, the use of a single instrumental within site case study means that these findings have limited transferability to a broader population for men in nursing or even other cohorts within the PNMU. Finally, an opportunity to corroborate Cliff and Jack’s experiences with the other female nursing students was missed as the women in the class were not interviewed.

\section{Conclusion}

This project explored the educational experience of male nursing students at PNMU. This is an important line of inquiry as a growing body of literature has identified that men face barriers in the education setting; however, there is limited research exploring when these barriers occur, what factors contribute to male student perceptions of barriers that do exist, and whether faculty are aware of barriers when they occur and are taking action to minimize those barriers that may exist. 
The researcher studied the students and faculty in an introductory nursing course during the first term, in order to examine how male students interacted with each other, their female peers, and their faculty and to see how men functioned within the educational setting. The findings showed that the two male subjects felt connected and supported by their faculty and that they appreciated having another male in the class with them. These connections appeared to have been instrumental in helping the men's positive perception of their educational experience. However, they also identified some areas of discomfort in the educational setting, specifically when practicing clinical skills with female peers, feeling pressured to volunteer and show skin during in-class demonstrations, and anticipating that they would be excluded from certain clinical situations. The evidence also showed that the connections between male and female students in the classroom may take longer to establish, may create instances of exclusion for men, may cause discomfort in the clinical setting, and may play an important role in whether men have a positive learning experience. These findings show that gender differences are present in the educational setting and may cause men to experience nursing education differently from their female peers.

In addition, the faculty were aware of the need to use gender-inclusive language in the classroom, had an expectation that men would be in their cohort, and identified awareness of certain clinical settings where men might be unwelcome. However, they did not have any knowledge of the specific barriers that Cliff and Jack might have encountered and did not identify ways in which they might help minimize barriers that Cliff and Jack might experience beyond using gender-neutral language. 
New questions emerged from this study that could use further investigation, including the exploration of how male/female student relationships help or hinder the educational experience in nursing, how men as gender minorities may disengage from participating in the educational setting when feeling pressured to volunteer, and how to increase the awareness of gender barriers within the educational setting with nursing faculty. Finally, gender does appear to play a role within the educational setting for men in nursing, and continued diligence concerning instances of difference by faculty and administrators is needed to help ensure that an inclusive learning environment is created for men in nursing. 


\section{References}

Alfred, D., Yarbrough, S., Martin, P., \& Garcia, C. (2011). Gender and professional values: A closer look. Nursing Management, 42(1), 34-36

American Assembly for Men in Nursing. (2011). American Assembly for Men in Nursing: Future of nursing message. Our strength and vision for partnering with others. Retrieved from http://aamn.org/docs/aamn-position.pdf

American Association of Colleges of Nursing (ACCN). (1997). Leading initiatives: Diversity and equality of opportunity. Retrieved from http://www.aacn.nche.edu/publications/position/diversity-and-equality

American Association of Colleges of Nursing (AACN). (2011). Fact sheet: Enhancing diversity in the nursing workforce. Retrieved from https://www.aacn.nche.edu/media-relations/fact-sheets/enhancing-diversity

Andrist, L. C., Nicholas, P. K., \& Wolf, K. A. (2006). A history of nursing ideas. Sudbury, MA: Jones and Bartlett.

Anthony, A. S. (2004). Gender bias and discrimination in nursing education. Nurse Educator, 29(3), 121-125.

Anthony, A. S. (2006). Tear down the barriers of gender bias. Men in Nursing, 1(4), 4349.

Bartfay, W. J., Bartfay, E., Clow, K. A., \& Wu, T. (2010). Attitudes and perceptions towards men in nursing. The Internet Journal of Allied Health Sciences and Practice, 8(2), 1-7. 
Bell-Scriber, M. J. (2008). Warming the nursing education climate for traditional-age learners who are male. Nursing Education Perspectives, 29(3), 143-150.

Berman, A., Snyder, S. J., Kozier, B., \& Erb, G. (2008). Historical and contempory nursing practice. Fundamentals of ursing: Concepts, process, and practice $\left(8^{\text {th }}\right.$ ed.). , Upper Saddle River, NJ: Pearson Education.

Bowen, H. R., \& Carnegie Council on Policy Studies in Higher Education. (1977). Investment in learning: The individual and social value of American higher educaiton. San Francisco: Jossey-Bass.

Brady, M. S., \& Sherrod, D. R. (2003). Retaining men in nursing programs designed for women. Faculty Forum, 42, 159-162.

Brown, T., Williams, B., Boyle, M., Molloy, A., McKenna,L., Palermo, C.... Lewis, B. (2011). Communication styles of undergraduate health students. Nurse Education Today, 31(4), 317-322.

Buerhaus, P. I. (1999). Is a nursing shortage on the way? Learn what you can do to prepare for a new wave of demand for nurses. Nursing Management, 30, 2, 54-55.

Creswell, J. W. (2007). Qualitative inquiry and research design (2 ${ }^{\text {nd }}$ ed.). Thousand Oaks, CA: Sage.

Crow, S. M., Smith, S. A., \& Hartman, S. J. (2005). Attrition in nursing: Perspectives from the national survey of college graduates. The Health Care Manager, 24(4), 336-346. 
PERCEPTIONS OF MALE NURSING STUDENTS IN NURSING EDUCATION 164

Denhart, H. (2008). Deconstructing barriers: Perceptions of students labeled with learning disabilities in higher education. Journal of Learning Disabilities, 41(6), 483-497. doi: 10.1177/0022219408321151

Denzin, N. K., \& Lincoln, Y. S. (2005). The Sage handbook of qualitative research ( $^{\text {rd }}$ ed.). Thousand, Oaks, CA: Sage.

Donahue, P., \& Russac, P. A. (1985). Nursing: The finest art. The American Journal of Nursing 85(10), 1080-1085.

Dyck, J. M., Oliffe, J., Phinney, A., \& Garrett, B. (2009). Nursing instructors’ and male nursing students’ perceptions of undergraduate, classroom nursing education. Nurse Education Today, 29(6), 649-653. doi: 10.1016/j.nedt.2009.02.003

Evangelista, A., \& Sims-Giddens, S. (2008). Gender differences in discipline of nurses in Missouri. Western Journal of Nursing Research, 30(4), 501-514. doi: 10.1177/0193945907303302

Evans, J., \& Frank, B. (2003). Contradictions and tensions: Exploring relations of masculinities in the numerically female dominated nursing profession.. The Journal of Men's Studies, 11(3), 277-292.

Evans, J. A. (2002). Cautious caregivers: Gender stereotypes and the sexulization of men nurses' touch. Journal of Advanced Nursing, 40(4), 441-448.

Fairhurst, G. T., \& Snavely, B. K. (1983a). A test of the social isolation of male tokens. Academy of Management Journal, 26, 353-361. 
Fairhurst, G.T., \& Snavely, B.K. (1983b). Majority and token minority group relationships: Power acquisition and communication. Academy of Management Review, 8(2), 292-300.

Fleming, R., Berkowitz, B., \& Cheadle, A. D. (2005). Increasing minority representation in the health professions. The Journal of School Nursing, 21(1), 31-39. doi: $10.1177 / 10598405050210010701$

Floge, L., \& Merrill, D. M. (1986). Tokenism reconsidered: Male nurses and female physicians in a hospital setting. Social Forces, 64(4), 925-947.

Gaynor, L., Gallasch, T., Yorkston, E., Stewart, S., \& Turner, C. (2006). Where do all the undergraduate and new graduate nurses go and why? A search for empirical research evidence. The Australian Journal of Advanced Nursing, 24 (2), 26-32.

Grady, G. A., Stewardson, G. A., \& Hall, J. L. (2006). Faculty notions regarding caring in male nursing students. Journal of Nursing Education, 47(7), 314-323.

Gustafson, J. L. (2008). Tokenism in policing: An empirical test of Kanter's hypothesis. Journal of Criminal Justice, 36(1), 1-10. doi: 10.1016/j.jcrimjus.2007.12.001

Harding, T., North, N., \& Perkins, R. (2008). Sexualizing men's touch: Male nurses and the use of intimate touch in clinical practice. Research and Theory for Nursing Practice, 22(2), 88-102.

Heikes, E. J. (1991). When men are the minority: The case of men in nursing. The Sociological Quarterly, 32, 389-401.

Holyaoke, D. D. (2000). Gender issues in nursing. Nursing Standard, 14(25), 31. 
PERCEPTIONS OF MALE NURSING STUDENTS IN NURSING EDUCATION 166

Institute of Medicine. (2001). The future of nursing: leading change, advancing health. Washingtong, DC: National Academies Press.

Institute of Medicine. (2004). In the nation's compelling interest. Ensuring diversity in the health-care workforce. Washington, DC; National Academy of Sciences.

James, T., \& Cinelli, B. (2003). Exploring gender-based communication styles. Journal of School Health ,73(1), 41-42.

Jary, D., \& Jary, J. (1991). Collins dictionary of sociology. Glasgow, UK: Harper Collins. Jeffreys, M. R. (2007). Tracking students through program entry progression, graduation, and licensure: Assessing undergraduate nursing student retention and success. Nurse Education Today, 27,406-419. doi: 10.1016/j.nedt.2006.07.003/c

Jinks, A. M. (1993). A study of attitudes to gender and nursing stereotypes. Nurse Education Today, 13, (4), 287-294.

Jinks, A. M. (2004). Angel, handmaiden, battleaxe, or whore? A study which examines changes in newly recruited student nurses’ attitudes to gender and nursing stereotypes. Nurse Education Today, 24(2), 121-127.

Johnson, B., \& Christensen, L. (2012). Educational research quantiative, qualitative, and mixed approaches ( $4^{\text {th }}$ ed.). Thousand Oaks, CA: Sage.

Johnson and Johnson. (2011). The campaign for nursing's future. Johnson \& Johnson Services, Inc. Retrieved from: http://www.discovernursing.com/

Judd, D., Sitzman, K., \& Davis, G. M. (2010). A history of American nursing: Trends and eras. Sudbury, MA: Jones and Bartlett. 
PERCEPTIONS OF MALE NURSING STUDENTS IN NURSING EDUCATION 167

Kanter, R. M. (1977). Some effects of proportions on group life: Skewed sex ratios and responses to token women. American Journal of Sociology, 82(5), 965-990.

Kelly, N. R., Shoemaker, M., \& Steele, T. (1996). The experience of being a male student nurse. Journal of Nursing Education, 35, 170-174.

Kenny, G. (2002). Male teacher and female students. Nurse Education Today, 22, 571577.

Kermode, S. (2006). Is nurse education sexist? An exploratory study. Contemporary Nurse, 22(1), 66.

Kippenbrock, T. (1990). School of nursing variables related to male student college choice. The Journal of Nursing Education, 29, 3, 118-21.

Kulackac, O., Ozkan, I. A., Sucu, G., \& O'Lynn, C. (2009). Nursing: The lesser of two evils for men. Nurse Education Today, 29, (6), 676-680.

Lerardi, J. A., Fitzgerald, D. A., \& Holland, D. T. (2010). Exploring male students' educational experiences in an associate degree nursing program. Journal of Nursing Education, 49(4), 215-218.

Love, K. L. (2010). The lived experience of socialization among African American nursing students in a predominantly white university. Journal of Transcultural Nursing, 21(4), 342-350. doi: 10.1177/1043659609360711

Mackintosh, C. (1997). A historical study of men in nursing. Journal of Advanced Nursing, 26, 232-236.

MacIntosh, J. (2002). Gender-related influences in nursing education. Journal of Profressional Nursing, 18(3), 170-175. 
PERCEPTIONS OF MALE NURSING STUDENTS IN NURSING EDUCATION 168

MacWilliams, B. R., Schmidt, B., \& Bleich, M. R. (2013). Men in nursing:

Understanding the challenges men face working in this predominantly female profession. American Journal of Nursing, 113(1), 38-44.

Maggs, C. (1996). A history of nursing: A history of caring? Journal of Advanced Nursing, 23, 630-635.

Marshall, C., \& Rossman, G. B. (2011). Designing qualitative research (5 ${ }^{\text {th }}$ ed.). Thousand Oaks, CA: Sage.

McLaughlin, K., Muldoon, O. T., \& Moutray, M. (2010). Gender, gender roles and completion of nursing education: A longitudinal study. Nurse Education Today, 30(4), 303-307. doi: 10.1016/j.nedt.2009.08.005

Meadus, R. J. (2000). Men in nursing: Barriers to recruitment. Nursing Forum, 35(3), 512.

Meadus, R. J., \& Twomey, J. C. (2011). Men student nurses: The nursing education experience. Nursing Forum, 46(4), 269-279.

Merriam, S. (1988). Case study research in education: A qualitative approach. San Francisco, CA: Jossey-Bass.

Merton, R. K., Fiske, M., \& Kendall, P. L. (1990). The focused interview: A manual of problems and procedures ( $2^{\text {nd }}$ ed.). New York: Free Press.

Nnedu, C. C. (2009). Recruiting and retaining minorities in nursing education. The ABNF Journal, 20(4), 93-96. 
PERCEPTIONS OF MALE NURSING STUDENTS IN NURSING EDUCATION 169

O'Lynn, C. (2004). Genderbased barriers for male students in nursing education programs: Prevalence and perceived importance. Journal of Nursing Education, 43(5), 229-236.

O'Lynn, C. (2009). Who is in need of socialization? Journal of Nursing Education, 48(4), 179.

Okrainec, G. D. (1994). Perceptions of nursing education held by male nursing students. Western Journal of Nursing Research, 16(1), 94-107. doi:

$10.1177 / 019394599401600108$

Oregon Center for Nursing. (2011). Oregon's nurse faculty workforce 2011. Retrieved from http://www.oregoncenterfornursing.org/documents/2011FacultyReport.pdf

Oregon Health Authority. (2011). Oregon health professions: Occupational and county profiles. Office for Oregon Health Policy and Research.

Paterson, B. L., Tschikota, S., Crawford, M., Saydak, M., Venkatesh, P., \& Aronowitz, T. (1996). Learning to care: Gender issues for male nursing students. The Canadian Journal of Nursing Research, 28(1), 25-39.

Patton, M. Q. (1990). Qualitative evaluation and research methods ( $2^{\text {nd }}$ ed.). Newbury Park, CA: Sage.

Poliafico, J. K. (1998). Nursing's gender gap. $R N$, 61(10), 39-42.

Rankin, S. R., \& Reason, R. D. (2005). Differing perceptions: How students of color and white students perceive campus climate for underrepresented groups. Journal of College Student Development, 46(1), 43-61. doi: 10.1353/csd.2005.0008 
Roth, J. E., \& Coleman, C. L. (2008). Perceived and real barriers for men entering nursing: Implications for gender diversity. Journal of Cultural Diversity, 15(3), 148-152.

Roter, D., Lipkin M. J., \& Korsgaard, A. (1991). Sex differences in patients' and physicians' communication during primary care medical visits. Medical Care, 29(11), 1083-93.

Schaefer, R. T. (2002). Racial and ethnic groups: Census 2000. Upper River, N.J.: Prentice Hall.

Segal, B. E. (1962). Male nurses: A case study in status contradiction and prestige loss. Social Forces, 41, 1, 31-38.

Shelton, E. N. (2003). Faculty support and student retention. Journal of Nursing Education, 42(2), 68-76.

Shelton, E. N. (2012). A model of nursing student retention. International Journal of Nursing Education Scholarship, 9(1), 1-16.

Smith, J. S. (2006). Exploring the challenges for nontraditional male students transitioning into a nursing program. Journal of Nursing Education, 45(7), 263269.

Smith, S. A., Crow, S. M., \& Hartman, S. J. (2007). Attrition in nursing among black and white nurses. Journal of Nursing Management 15, 357-366.

Snavely, B. K. (1983). A test of the social isolation of male tokens. Academy of Management Journal, 26, 353-361.

Stake, R. (1995). The art of case study research. Thousand Oaks, CA: Sage. 
Stickney, M. C. (2008). Factors affecting practical student attrition. Journal of Nursing Education, 47(9), 422-425.

Stott, A. (2003). Issues in the socialisation process of the male student nurse: Implications for retention in undergraduate nursing courses. Nurse Education Today, 24, 91-97. doi: 10.1016/j.nedt.2003.09.005/c

Stott, A. (2006). Exploring factors affecting attrition of male students from an undergraduate nursing course: A qualitative study. doi: 10.1016/j.nedt.2006.05.013/c

Street, R. L. (2002). Gender differences in health care provider-patient communication: Are they due to style, stereotypes, or accomodation? Patient Education and Counseling 48(3), 201-206.

Streubert, H. J. (1994). Male nursing students' perceptions of clinical experience. Nurse Educator, 19(5), 28-32.

Stroshine, M. S., \& Brandl, S. G. (2011). Race, gender, and tokenism in policing: An empirical elaboration. Police Quarterly, 14(4), 344-365. doi: $10.1177 / 1098611111423738$

Sullivan, L. W. (2004). U.S. health-care professions separate and unequal. Black Issues in Higher Education, 21(17),11.

Sullivan, L. W. (2005). Health report: The Sullivan Alliance partners with Joint Center for Political and Economic Studies to increase diversity in the health professions. Focus, 33(5), 1-16. 
PERCEPTIONS OF MALE NURSING STUDENTS IN NURSING EDUCATION 172

The Sullivan Commission. (2010). Missing persons: Minorities in the health professions. A report of the Sullivan Commission on diversity in the healthcare worforce.

Retrieved from http://depts.washington.edu/ccph/pdf_files/Sullivan_Report_ES.pdf.

Thomas, S. P. (1998). Transforming nurses' anger and pain: Steps toward healing. New York: Springer.

Ting-Toomey, S. (1999). Communicating across cultures. New York: Guilford Press.

U.S. Department of Commerce Economics and Statistics Administration. (2010a). American community survey. United States Census Bureau. Retrieved from http://quickfacts.census.gov/qfd/states/00000.html

U.S. Department of Commerce Economics and Statistics Administration. (2010b). State profile. United States Census Bureau. Retrieved from http://quickfacts.census.gov/qfd/states/41000.html

U.S. Department of Health and Human Services, Health Resources and Services Administration. (2000). National sample survey of registered nurses. Washington, D.C.

U.S. Department of Health and Human Services, Health Resources and Services Administration. (2004). National sample survey of registered nurses. : Washington, D.C.

U.S. Department of Health and Human Services, Health Resources and Services Administration. (2008). National sample survey of registered nurses. : Washington, D.C. 
PERCEPTIONS OF MALE NURSING STUDENTS IN NURSING EDUCATION 173

U.S. House of Representatives. (2010). Compilation of patient protection and affordable care act. U.S. House of Representatives. 111th Congress 2nd Session. Public Law 111-148.

Vogt, C. M., Hocevar, D., \& Hagedorn, L. S. (2007). A social cognitive construct validation: Determining women's and men's success in engineering programs. The Journal of Higher Education, 78(3), 337-364.

Williams, M. G. (2010). Attrition and retention in the nursing major: Understanding persistence in beginning nursing students. Nursing Education Perspectives, 31(6), $362-367$.

Wingfield, A. H. (2009). Racializing the glass elevator: Reconsidering men's experiences with women's work. Gender \& Society, 23(1), 5-26. doi:

$10.1177 / 0891243208323054$

Woods, R. A. (2009). Industry output and employment projections to 2018. Monthly Labor Review,132(11), 52-81.

Yang, C. I., Gau, M. L., Shiau, S. J., Hu, W. H., \& Shih, F. J. (2004). Professional career development for men in nursing. Nursing and Health Care Management and Policy, 48(6), 642-650.

Yin, R. K. (2009). Case study research: Design and method ( $4^{\text {th }}$ ed.). Thousand Oaks, CA: Sage. 


\section{Appendices}

\section{Appendix A: Case Study Design Graphic}

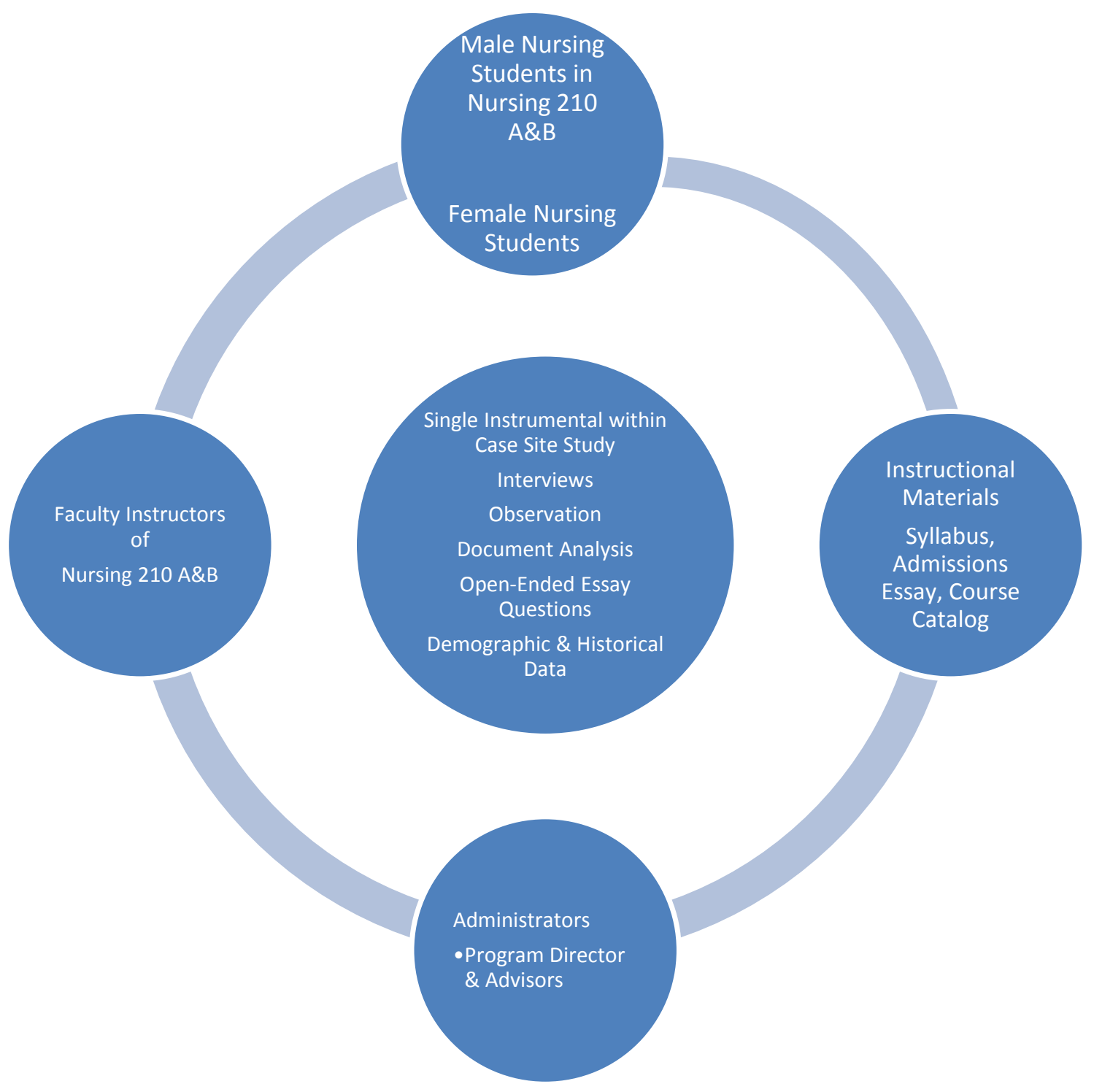




\section{Appendix B: All Data Collection Timeline}

\section{Proposed Study Implementation Timeline}

\begin{tabular}{|c|c|}
\hline Prior to start of fall term & $\begin{array}{l}\text { - Meet with lead faculty member of } \\
\text { Nursing } 210 \text { course and get consent to } \\
\text { participate and observe the class. }\end{array}$ \\
\hline 1st week of fall term & $\begin{array}{l}\text { - Meet with Nursing } 210 \text { class - review } \\
\text { the study - get consent forms. } \\
\text { - Recruit men from the class to participate } \\
\text { in the research project and get consent } \\
\text { forms. } \\
\text { - Request participation from program } \\
\text { director and male nursing student } \\
\text { advisors and get consent forms. }\end{array}$ \\
\hline 2nd week of fall term & $\begin{array}{l}\text { - Observe Nursing } 210 \text { A\& B classes for } \\
\text { an hour each and take notes. } \\
\text { - Send open-ended essay question \#1 to } \\
\text { faculty and male nursing student } \\
\text { participants. } \\
\text { - Interview male nursing students. }\end{array}$ \\
\hline 5th week of fall term & $\begin{array}{l}\text { - Observe Nursing } 210 \text { A\& B classes for } \\
\text { an hour each and take notes. } \\
\text { - Send open-ended essay question \#2 to } \\
\text { faculty and male nursing student } \\
\text { participants. }\end{array}$ \\
\hline 10th week of fall term & $\begin{array}{l}\text { - Observe Nursing } 210 \text { A\& B classes for } \\
\text { an hour each and take notes. } \\
\text { - Send open-ended essay question \#3 to } \\
\text { faculty and male nursing student } \\
\text { participants. } \\
\text { - Interviews with program director and } \\
\text { male nursing student advisors. }\end{array}$ \\
\hline 11th week of fall term & $\begin{array}{l}\text { - Focused interviews with male nursing } \\
\text { students. } \\
\text { - Focused interviews with faculty } \\
\text { members. }\end{array}$ \\
\hline 12th week of fall term & - End of study. \\
\hline
\end{tabular}




\section{Revised Study Implementation Timeline Due to IRB Approval}

\begin{tabular}{|c|c|}
\hline 2ndweek of fall term & $\begin{array}{l}\text { - Met with lead faculty member of } \\
\text { Nursing } 210 \text { course, get consent to } \\
\text { participate and observed the class. } \\
\text { - } \\
\text { Requested participation from program } \\
\text { director and reviewed consent forms. }\end{array}$ \\
\hline 3rd week of fall term & $\begin{array}{l}\text { - Met with Nursing } 210 \text { class - reviewed } \\
\text { the study, got consent forms for } \\
\text { observation. } \\
\text { - Sent recruitment e-mails to all students } \\
\text { in the class to request participation in } \\
\text { the study and review informed consent. } \\
\text { - Observed Nursing } 210 \text { A classes for } 2 \\
\text { hours and take notes. }\end{array}$ \\
\hline 4th week of fall term & $\begin{array}{l}\text { - Lead faculty Susan administered the } \\
\text { first in-class research activity to Nursing } \\
210 \text { A students at end of class. }\end{array}$ \\
\hline 5th week of fall term & $\begin{array}{ll}\text { - } & \text { Observed Nursing } 210 \text { A class for } 2 \\
\text { hours. } \\
\text { - } \\
\text { Observed Nursing } 210 \text { B classes for } 4 \\
\text { hours. }\end{array}$ \\
\hline 6th week of fall term & $\begin{array}{ll}\text { - } & \text { Mid-term interview with Cliff. } \\
\text { - } & \text { Mid-term interview with Jack. }\end{array}$ \\
\hline 7th week of fall term & $\begin{array}{ll}\text { - } & \text { Observed Nursing } 210 \text { A class for } 2 \\
\text { hours. } \\
\text { - } \\
\text { Observed Nursing } 210 \text { B classes for } \\
\text { 2hours. }\end{array}$ \\
\hline 11th week of fall term & $\begin{array}{l}\text { - Lead faculty Susan administered the } 2^{\text {nd }} \\
\text { in-class research activity to Nursing } 210 \\
\text { A students at end of class. } \\
\text { - } \quad \text { Observed Nursing } 210 \text { A for } 2 \text { hours. }\end{array}$ \\
\hline 12th week of fall term & $\begin{array}{l}\text { End of term Interviews with Faculty } \\
\text { members Susan \& Marcie. } \\
\text { - } \quad \text { End of term interviews with Program } \\
\text { Administrator Vickie. } \\
\text { - } \quad \text { End of term interview with Cliff. }\end{array}$ \\
\hline $1^{\text {st }}$ week of Winter term & 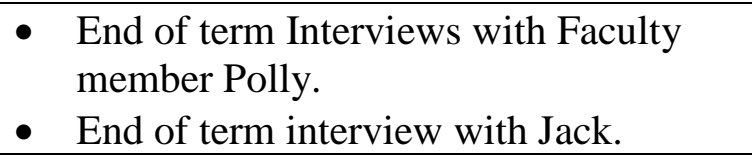 \\
\hline
\end{tabular}




\section{Appendix C: Emails for recruitment participation}

Example E-mail or conversation with lead faculty member of Nursing 210 A\&B:

Hello Dr. X.,

I am a doctoral student in the EdD for Higher Education and Leadership program at Portland State University. As part of my research, I am conducting a study about men in nursing and I would like to observe your Nursing 210 A\&B classes this fall term. I chose Nursing 210 A\&B as it is the first class that all new nursing students take, and I believe that this course is a great class to get a good sense of the academic and professional expectations that will be shared with new nursing students. I would plan to observe during weeks 2, 5, and 10, and I would also be asking to interview you and any other faculty members associated with the class at the end of the term. In addition, I would be asking for your participation with responding to three short answer essay questions throughout the term about your perceptions and experiences of the participants in the class. As part of this study, I will also be recruiting the male nursing students to interview at the beginning and end of the term and will also be looking for them to respond to three short-answer essay questions throughout the term. Your responses will remain confidential with any information that you share being used without any identifying information from you in the final report. As a nursing faculty, you have a unique view on the transition that men make into nursing school and your participation in this research study will be truly beneficial in helping us learn more about the experiences of men in our nursing program. I appreciate your participation. 
Example email invitation for male nursing student participation:

Your participation needed!

Dear,

I am a doctoral student in the EdD for Higher Education and Leadership program at Portland State University. As part of my research, I am conducting a study about men in nursing and I would like to enlist you as a participant in this research. This term I will be observing your nursing course 210 A\&B and I would also like to interview you and have you respond to some open-ended essay questions during your first term. Your participation would consist of two one-hour interviews during the 2nd and 10th weeks of the term as well as your time with responding to 3 short essay questions during the 2nd, 5th, and 10th weeks of the term. Your participation is voluntary, and your academic progression and grade in this course will not be influenced by your participation in this research or lack thereof. However, if you do choose to participate, your experiences as a student will remain confidential with any information that you share being used without any identifying information from you in the final report. As a male nursing student, your ideas, perceptions, and experiences in nursing school are an integral factor in this research and your participation will assist us with better understanding the needs and experiences of our students.

If you are interested in participating, please contact Jennifer Anderson at jander2@pdx.edu by XX date. I look forward to having you join this important research study. 
PERCEPTIONS OF MALE NURSING STUDENTS IN NURSING EDUCATION 179

Email invitation for program director and male nursing student advisor interview:

Dear Dr. X.,

I am conducting a research study where I am looking at the ways that men transition into nursing school and I have received permission from Dr. X to observe Nursing 210 A\&B and to interview the male nursing students participating in that class. As part of this process, I would also like to interview you at the end of the term to gain a broader understanding of this topic. I would request one hour of your time at the end of fall term and will set up an appointment to meet with you in person to discuss this further.

If you are interested in participating, please contact Jennifer Anderson at jander2@pdx.edu by XX date. I look forward to having you join this important research study. 


\section{Appendix D: Interview Questions}

Interview questions for male nursing students at the start of term:

1. Tell me about your interest in becoming a nurse.

2. Did you have any concerns about being a man in the nursing profession?

3. Before you started nursing school, did you know any other male nurses?

a. Have you seen/met any male nurses since you started nursing school?

4. How would you say your transition into nursing school has gone so far?

a. What are your expectations for your first term in nursing school?

b. Are you concerned or worried about any of your experiences so far?

5. Have you noticed anything different about being a student in this educational setting than others you have been in before?

6. How would you describe your interactions with the other students in your cohort so far?

a. Have you bonded with any students in particular?

b. Have you noticed if you have been treated differently from any of the other students?

c. Have you noticed if others seem more aware of you than the other students in the class? If so, why do you think that is?

7. How would you describe your interactions with the faculty in your classes so far?

a. Have you noticed if you have been treated differently from any of the other students? 
b. Have you noticed if your faculty seem more aware of you than the other students in the class? If so, why do you think that is?

8. How would you describe the nursing curriculum so far?

a. Do you find that you can relate to the examples in class? Why, why not?

b. Who are the role models in nursing that have been identified so far?

c. Have you encountered any examples or discussions that seemed more applicable to women than men?

d. What are you hoping to learn from your classes this term?

e. Is there anything that you are nervous about learning or practicing clinically?

9. How would you describe your experience as a student at this institution so far?

a. Have you noticed if you have been treated differently from any of the other students?

b. Have you noticed if your administrators seem more aware of you than the other students in the class? If so, why do you think that is?

Interview questions for male nursing students at the end of term:

1. Tell me about how your first term of nursing school.

a. Were you surprised by anything that you learned?

b. When you think back to the start of the term, do you feel like your original expectations were met? 
c. Did you experience any challenges as a male nursing student?

2. Who would you describe as a role model in nursing for you?

3. Have you noticed anything different about being a student in this educational setting than others you have been in before?

4. How would you describe your interactions with the other students in your cohort this term?

a. Have you bonded with any students in particular?

b. Have you noticed if you have been treated differently from any of the other students?

c. Have you noticed if others seem more aware of you than the other students in the class? If so, why do you think that is?

5. How would you describe your interactions with the faculty this term?

a. Have you noticed if you have been treated differently from any of the other students?

b. Have you noticed if your faculty seem more aware of you than the other students in the class? If so, why do you think that is?

c. Would you say you were called on more or less than others in your class this term?

6. How would you describe the nursing curriculum so far?

a. Do you find that you can relate to the examples in class? Why, why not?

b. Have you encountered any examples or discussions that seemed more applicable to women than men or vice versa? 
c. Would you describe your learning experience as the same as the others in your class? If not, how was it different?

7. How would you describe your experience as a student at this institution so far?

a. Did you find opportunities to become involved in activities outside of class?

8. Are you aware of any barriers within the educational setting that may make it more difficult for men?

Interview questions for faculty at the end of term:

1. Describe the members of the class you taught this term.

a. How did the students do overall this term?

b. Were you surprised by any of the dynamics within the class?

2. Did you notice or find anything different about this group of students than others you have taught before?

3. How would you describe your interactions with the students in your class this term?

a. Have you bonded with any students in particular?

b. Have you noticed if any students were treated differently from any of the other students? If so, why do you think that is?

c. Have you noticed if any students seemed to stand out more than other students in the class? If so, why do you think that is?

4. How would you describe the male students in your class this term? 
5. Did you ever notice if the male nursing students felt uncomfortable with any learning activities?

6. Did you ever witness male nursing students being excluded from groups or learning activities?

7. Did you ever change a teaching example to help illustrate something for the male nursing students that would have been more obvious to the female nursing students?

8. In your experience, are there any differences in the way that female and male nursing students acclimate to the nursing culture?

9. In your experience, do you find any differences in the way that men and women approach the nursing curriculum?

10. In your experience, do you find that men have difficulties with certain aspects of the nursing role?

11. Have you ever found yourself changing your educational style to accommodate the perceived learning needs of men?

12. Have you ever witnessed gender stereotypes for men in nursing?

13. What, if any, barriers are there for men in nursing?

14. Are you aware of any barriers within the educational setting that may make it more difficult for men?

Interview questions for administrator at end of term:

1. Please describe your role related to Nursing 210. 
2. What do you hope this course will provide for these students?

3. Would you say that this course broadens the student perspective about the role of a nurse?

4. Do you ever see any differences between how men and women approach learning in this course?

5. Have you ever noticed any differences in the way that men approach nursing school compared to their female peers?

6. Do you think that students are socialized to nursing during the first term?

7. Are you aware of any barriers within the educational setting that may make it more difficult for men?

8. Would you say that people are open and accepting of the people who are in their classrooms?

9. Do you think the faculty in this program are aware of barriers that may exist for men in nursing?

10. Do you believe that the faculty in this program value an inclusive learning environment for men?

11. What if any preparation do students receive in anticipation of being excluded from specific clinical sites?

12. Were you aware of any faculty who struggled with teaching this course this term 


\section{Appendix E: Code Graphic}

\section{Not Aware of Gender Differences.}

Nursing Culture at OHSU

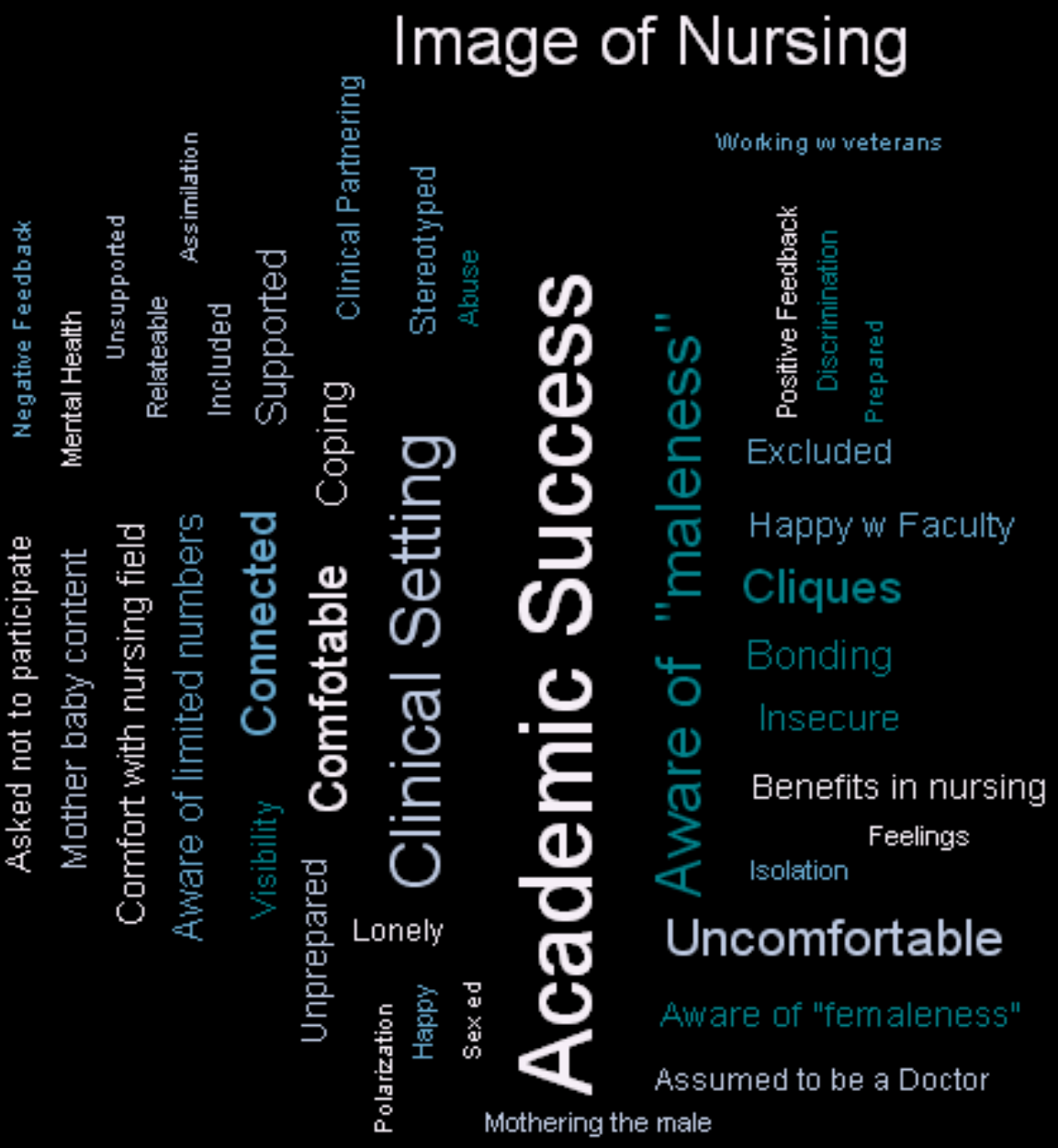

Aware of Gender Differences?

\section{How Nurses Think}

